Juliana Maria da Silva

\title{
MODELAGEM NUMÉRICA DO ESCOAMENTO NUM TUBO PERMEÁVEL APLICADA AO PROCESSO DE FILTRAÇÃO TANGENCIAL
}

Tese apresentada à Escola de Engenharia de

São Carlos da Universidade de São Paulo como parte dos requisitos para obtenção do título de Doutora em Engenharia Mecânica.

Orientador: prof. Dr. Sergio Rodrigues Fontes 
Aos meus pais João e Maria Olga 


\section{Agradecimentos}

Em primeiro gostaria de agradecer a Deus pela minha vida e aos meus pais, por todo apoio e compreensão nos momentos mais difíceis desse trabalho.

Ao prof. Dr. Sergio Rodrigues Fontes pelo apoio, orientação e incentivo. Ao prof. Dr. Valdemir Garcia Ferreira pela boa vontade em ajudar e dar sugestões.

Ao Conselho Nacional de Desenvolvimento Científico e Tecnológico (CNPQ) pelo apoio financeiro para a realização desta pesquisa (processo: 142116/2003-3), a Coordenação de Aperfeiçoamento de Pessoal de Nível Superior (CAPES) e a Fundação de Amparo à Pesquisa do Estado de São Paulo (FAPESP).

A todos os colegas do NETeF, de modo especial agradeço aos amigos Roberta, Renata e Ernesto pela amizade e companheirismo e ao amigo Venezuela pela amizade e por toda a ajuda. E todos que indiretamente colaboraram na realização desse trabalho. 


\section{Resumo}

SILVA, J.M. (2008). Modelagem numérica do escoamento num tubo permeável aplicada ao processo de filtração tangencial. Tese (Doutorado) - Escola de Engenharia de São Carlos, Universidade de São Paulo, São Carlos, 2008.

Estudos aplicados ao processo de filtração tangencial têm sido o objetivo de muitos trabalhos devido a sua ampla capacidade de adequação aos mais diversos processos industriais. Os mecanismos de transferência associados a esse processo envolvem basicamente o escoamento em tubos permeáveis (ou membranas) com fenômenos de transferência de massa presentes junto à superfície. Esta tese de doutorado apresenta uma modelagem numérica capaz de representar o escoamento de fluidos newtonianos e não-newtonianos em tubos permeáveis aplicada ao processo de filtração tangencial, para os regimes de escoamento laminar e turbulento. No regime turbulento, utilizou-se o modelo de comprimento de mistura de Prandtl. O modelo numérico envolveu as equações de conservação da massa, da quantidade de movimento e das espécies químicas, acopladas a adequadas condições de fronteira. Em particular, por se tratar de um modelo numérico, analisou-se a discretização dos termos convectivos por meio da implementação de três esquemas: WACEB, CUBISTA e QUICKEST adaptativo. Os resultados produzidos pela implementação dos esquemas 
convectivos foram analisados por meio de comparações com resultados analíticos e experimentais da literatura, para regimes de escoamento laminar e turbulento. De acordo com as comparações realizadas, o esquema QUICKEST adaptativo apresentou melhor desempenho na modelagem desse escoamento. Diversas simulações numéricas geraram resultados, os quais foram comparados com expressões analíticas e dados experimentais da literatura e com dados produzidos pelo laboratório do Núcleo de Engenharia Térmica e Fluidos (NETeF) da USP/São Carlos. Verificou-se, assim, que o modelo matemático produziu resultados compatíveis com o fenômeno estudado, tendo-se, portanto, uma ferramenta para descrição do problema convectivo mássico do escoamento em tubos permeáveis.

Palavras-chave: filtração tangencial; membrana tubular; fluido não-newtoniano; modelagem matemática 


\section{Abstract}

SILVA, J.M. (2008). Numerical modeling of the flow in permeable tube applied to the crossflow filtration process. Ph.D. Thesis - Escola de Engenharia de São Carlos, Universidade de São Paulo, São Carlos, 2008.

Studies applied to the crossflow filtration process have been the goal of several researches due to its large capacity for adaptation to the various industrial processes. The mechanism of transfer associated with this process basically involves flows in permeable tubes (or membranes) with the phenomenon of mass transfer on the surface. This doctoral thesis presents a numerical modeling able to represent the flow of Newtonian and non-Newtonian fluids in permeable tubes applied to the process of crossflow filtration, in laminar and turbulent flow regimes. In the turbulent regime the Prandtl mixing length model was used. The numerical model involved the mass conservation, momentum conservation and mass transport equations coupled to the appropriate boundary conditions. In particular, as it is a numerical model, the discretization of convective terms was analyzed through the implementation of three schemes: WACEB, CUBISTA and adaptative QUICKEST. The results produced by the implementation of the convective schemes were analyzed through comparisons with experimental and analytical results from the literature, for laminar and turbulent flow 
regimes. According to the comparisons made, adaptative QUICKEST scheme showed the best performance in the modeling in this flow. Several numerical simulations generated results, which were compared with experimental data and analytical expressions of the literature and with data produced by the laboratory of the Center of Thermal Thermal and Fluids Engineering (NETeF) of the EESC-USP/São Carlos. It was possible to verify, that the mathematical model produced results consistent with the phenomenon studied, and can be considered a tool for the description of the mass convective problem flow in permeavel tubes.

Keywords: crossflow filtration; tubular membrane; non-Newtonian fluid; mathematical modeling 


\section{Lista de Figuras}

2.1 Esquema de filtração tangencial. . . . . . . . . . . . . . . . . . . . . 6

2.2 Concentração de polarização. . . . . . . . . . . . . . . . . . 7

2.3 Volume de controle na camada de polarização. . . . . . . . . . . . . . . . . 13

2.4 Esquema de classificação dos fluidos, segundo comportamento reológico. . . 18

3.1 Representação do domínio do problema. . . . . . . . . . . . . . . . . . . 28

3.2 Camada de polarização. . . . . . . . . . . . . . . . . . . . . . 43

4.1 Célula computacional da malha deslocada. . . . . . . . . . . . . . 46

4.2 Célula adjacente à parede porosa. . . . . . . . . . . . . . . . . 53

4.3 Representação esquemática para se obter aproximações dos termos convectivos. 56

5.1 Perfil de velociadade axial em função da coordenada radial para os diferentes esquemas convectivos com uma malha computacional de dimensões 400x40. 76

5.2 Perfil de velocidade axial em função da coordenada radial para diferentes malhas computacionais. . . . . . . . . . . . . . 78

5.3 Perfil da queda de pressão em função da coordenada $z /(R \times R e)$. . . . . . 80

5.4 Número de Sherwood em função da coordenada axial. . . . . . . . . . . . . . 83 
5.5 Concentração próxima à superfície permeável em função da coordenada axial, para números de Reynolds 100 e 300. . . . . . . . . . . . . . . . . . . 85

5.6 Número de Sherwood médio em função de $n$, para números de Reynolds 150

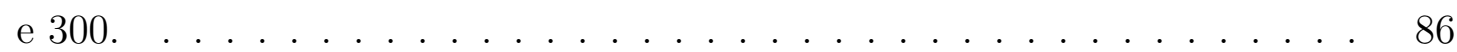

5.7 Critério de parada em função do número de iterações. . . . . . . . . . . . . 90

5.8 Concentração próxima à superfície da membrana em função da coordenada

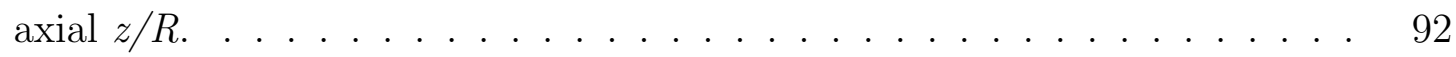

5.9 Velocidade de permeação média em função da pressão transmembrana inicial. 93

5.10 Velocidade de permeação média de uma solução de Dextran T500 em função da pressão transmembrana inicial. . . . . . . . . . . . . . . . . . . . . 96

5.11 Velocidade de permeação média de uma solução de PVP-360 em função da pressão transmembrana inicial. . . . . . . . . . . . . . . . .

5.12 Concentração local em função da coordenada radial para diferentes localizações axiais. . . . . . . . . . . . . . . . . . . . . . . . . . . 98

5.13 Concentração local próxima à superfície da membrana em função da coordenada radial para diferentes pressões transmembrana inicial. . . . . . . . .

5.14 Concentração local em função da coordenada axial para diferentes números de Reynolds. . . . . . . . . . . . . . . . . . . . . . . . . 100

5.15 Concentração local em função da coordenada radial para diferentes números de Reynolds. . . . . . . . . . . . . . . . . . . . . . . . . 101

5.16 Número de Sherwood em função da coordenada axial para diferentes números de Reynolds. . . . . . . . . . . . . . . . . . . . . . . . . . . . 101

5.17 Concentração próxima à superfície da membrana em função da coordenada axial, para diferentes pressões transmembrana. . . . . . . . . . . . . 103 
5.18 Velocidade de permeação média em função da pressão transmembrana inicial.103

5.19 Velocidade de permeação média em função do número de Reynolds. . . . . 105

5.20 Velocidade média de permeação em função da pressão transmembrana inicial.107

5.21 Número de Sherwood médio em função do número de Reynolds. . . . . . . 108

5.22 Número de Sherwood médio em função do número de Reynolds. . . . . . . 109

5.23 Comparação do número de Sherwood local em função da coordenada axial. 111 


\section{Lista de Tabelas}

5.1 Erro relativo máximo referente às simulações representadas na Fig. 5.5. . . 85

5.2 Erro relativo referente às simulações representadas na Fig. 5.6 para $\operatorname{Re}=150.87$

5.3 Erro relativo referente às simulações representadas na Fig. 5.6 para $\operatorname{Re}=300.87$

5.4 Número de iterações referente às simulações representadas na Fig. 5.8. . . . 91

5.5 Número de iterações referente às simulações representadas na Fig. 5.9. . . . 93 


\section{Lista de Símbolos}

$c \quad$ concentração de soluto $(w t . \%)$

$c_{0}$ concentração de alimentação $(w t . \%)$

$c_{g} \quad$ concentração na camada gel $(w t . \%)$

$c_{w} \quad$ concentração na superfície da parede porosa (wt.\%)

$d \quad$ diâmetro do tubo $(m)$

$D \quad$ coeficiênte de difusão $\left(m^{2} s^{-1}\right)$

$D_{t} \quad$ coeficiente de difusão turbulento $\left(\mathrm{m}^{2} \mathrm{~s}^{-1}\right)$

g vetor aceleração da gravidade

I tensor identidade

$l \quad$ comprimento de mistura $(m)$

$L \quad$ comprimento da membrana tubular $(m)$

$L_{p} \quad$ permeabilidade $\left(\mathrm{m}^{2} s k g^{-1}\right)$

$m \quad$ coeficiente de consistência $\left(P a s^{n}\right)$

$n \quad$ índice de comportamento

$N \quad$ nível de tempo

$p \quad$ pressão $(P a)$

$p_{0} \quad$ pressão de entrada $(P a)$ 
$p_{\text {ext }} \quad$ pressão externa $(P a)$

$\Delta p \quad$ pressão transmembrana $(P a)$

$r \quad$ coordenada radial $(m)$

$\Delta r \quad$ tamanho da célula ao longo da direção r $(m)$

$R \quad$ raio do tubo $(m)$

$R_{m} \quad$ resistência da membrana $\left(k g m^{-2} s^{-1}\right)$

$R_{f}$ resistência devido a camada de concentração de polarização $\left(\mathrm{kgm}^{-2} \mathrm{~s}^{-1}\right)$

$t \quad$ tempo $(s)$

$\Delta t \quad$ passo no tempo $(s)$

$u \quad$ velocidade axial $\left(m s^{-1}\right)$

$u_{0} \quad$ velocidade axial de entrada $\left(m s^{-1}\right)$

$v \quad$ velocidade radial $\left(m s^{-1}\right)$

$v_{m} \quad$ velocidade média $\left(m s^{-1}\right)$

$v_{w} \quad$ velocidade de permeação local $\left(m s^{-1}\right)$

$\bar{v}_{w} \quad$ velocidade de permeção média $\left(m s^{-1}\right)$

$z \quad$ coordenada axial $(m)$

$\Delta z \quad$ tamanho da célula ao longo da direção z $(m)$

\section{Letras Gregas}

$\delta \quad$ espessura da camada limite de concentração $(m)$

$\varepsilon_{c}$ parâmetro de convergência para a concentração

$\varepsilon_{v} \quad$ parâmetro de convergência para a velocidade 
$\phi \quad$ constante de proporcionalidade $\left(\mathrm{sm}^{-1}\right)$

$\dot{\gamma} \quad$ taxa de deformação

$\overline{\bar{\gamma}} \quad$ tensor taxa de deformação

$\eta \quad$ viscosidade aparente $\left(\mathrm{Pas}^{-1}\right)$

$\kappa \quad$ coeficiente de transferência de massa

$\kappa_{\text {local }}$ coeficiente de transferência de massa local

$\mu \quad$ viscosidade dinâmica $\left(\mathrm{Pas}^{-1}\right)$

$\mu_{t} \quad$ viscosidade dinâmica turbulenta $\left(\mathrm{kgm}^{-1} \mathrm{~s}^{-1}\right)$

$\Pi \quad$ pressão osmótica $(\mathrm{Pa})$

$\rho \quad$ massa específica $\left(\mathrm{kgm}^{-3}\right)$

$\sigma \quad$ tensor tensão total

$\tau \quad$ tensor tensão

$\tau_{w} \quad$ tensão de cisalhamento na parede

Números Adimensionais

Re número de Reynolds generalizado para fluidos não-newtonianos $\left(\frac{u_{0}^{2-n} d^{n} \rho}{m 8^{n-1}}\left(\frac{4 n}{3 n+1}\right)^{n}\right)$

Pe número de Peclet $\left(\frac{d u_{0}}{\mathbf{D}}\right)$

Sh número de Sherwood local $\left(\frac{-2 \frac{\partial c}{\partial r} \mid w}{c_{w}-1}\right)$

$\overline{S h}$ número de Sherwood médio 


\section{Sumário}

1 Introdução 1

2 Revisão Bibliográfica $\quad 5$

2.1 Conceitos básicos de filtração tangencial . . . . . . . . . . . . . . . 5

2.1.1 Determinação da concentração na superfície da membrana . . . . . 13

2.1 .2 Número de Sherwood . . . . . . . . . . . . . . . . . . . . . . . . . . 14

2.2 Reologia . . . . . . . . . . . . . . . . . . . 17

2.3 Equação reológica de estado . . . . . . . . . . . . . . . . . . . . . . . . . . 19

2.4 Modelagem . . . . . . . . . . . . . . . . . . . 20

3 Descrição da Modelagem Matemática 27

3.1 Descrição do domínio . . . . . . . . . . . . . . . . . . . . . . 27

3.2 Formulação matemática do escoamento laminar $\ldots$. . . . . . . . . . . . . . 28

3.2.1 Formulação matemática do escoamento turbulento . . . . . . . . . . 30

3.2 .2 Adimensionalização . . . . . . . . . . . . . . . . . . . . . . 40

3.2.3 Condições iniciais e de fronteira . . . . . . . . . . . . . . . . . . . . 41

4 Discretização das Equações e Método Computacional 45 
4.1 Discretização $\ldots \ldots \ldots \ldots \ldots \ldots$

4.1.1 Discretização das equações que modelam o escoamento . . . . . . . 47

$4.1 .2 \quad$ Aproximação dos termos convectivos . . . . . . . . . . . . . 54

4.2 Método numérico . . . . . . . . . . . . . . . . . . . . . . . . . . . . 67

4.3 Controle do passo no tempo $\ldots \ldots \ldots \ldots \ldots$

5 Resultados e Discussões $\quad 73$

5.1 Análise dos esquemas convectivos . . . . . . . . . . . . . . . . 74

5.1.1 Escoamento em tubos com paredes impermeáveis . . . . . . . . 74

5.1 .2 Escoamento em tubos com paredes permeáveis . . . . . . . . . . 79

5.2 Resultados com o esquema QUICKEST adaptativo . . . . . . . . . . . . 94

5.2.1 Resultados para escoamento em regime laminar . . . . . . . . . 94

5.2 .2 Resultados para escoamento em regime turbulento . . . . . . . . . 102

6 Conclusões e Sugestões de Trabalhos Futuros 113

$\begin{array}{ll}\text { Referências Bibliográficas } & 129\end{array}$

\begin{tabular}{ll}
\hline A Programa & 131
\end{tabular} 


\section{Capítulo 1}

\section{Introdução}

As investigações de escoamentos aplicados em engenharia podem ser realizadas por dois métodos: o método experimental e o método teórico. Ambos visam à descrição e a o entendimento dos fenômenos presentes no problema físico. O método experimental requer medições de grandezas fundamentais, as quais, podem requerer equipamentos de alto custo, cuja flexibilidade, muitas vezes, não seria o suficiente para permitir a descrição do problema em amplos regimes e intervalos de parâmetros significativos. O método teórico consiste em converter as leis de conservação apropriadas em relações matemáticas, empregando-se a linguagem do cálculo diferencial ou integral.

Quando as equações envolvem não-linearidades em suas formulações, as soluções analíticas são raramente encontradas, assim, os métodos numéricos oferecem uma ferramenta indispensável na determinação de soluções aproximadas (soluções numéricas). Esses métodos consistem em discretizar as equações desenvolvidas pelo método teórico e resolvêlas aproximadamente em pontos no espaço definidos pelo problema. A precisão da solução depende do número de pontos utilizados na simulação.

Assim, com algoritmos computacionais eficazes e com o surgimento de computa- 
dores cada vez mais rápidos e com maior capacidade de armazenamento de dados, as simulações numéricas, em muitos casos, tornaram-se mais próximas das soluções reais e, portanto, podem ser utilizadas para simular situações reais.

Por esse motivo, muitos processos industriais confiam agora no uso de modelos computacionais para auxiliar no processo de desenvolvimento. Exemplos clássicos do uso de ferramentas numéricas em processos industriais são os escoamentos de fluidos com convecção térmica, presentes na indústria de trocadores de calor e no desenvolvimento da indústria aeronáutica, na modelagem de perfis aerodinâmicos.

Nas últimas quatro décadas o processo de filtração tangencial com membranas tubulares tem sido largamente adotado por diferentes indústrias, como: nas de alimento, para clarificação de vinhos, sucos de frutas e vinagre, remoção de levedura de cerveja, separação de bactérias e gorduras do leite; nas indústrias químicas, no processamento de tintas e derivados do petróleo; na indústria farmacêutica e de cosméticos, para produção de água ultrapura; dentre outras. O processo tem potencial de expansão em setores importantes, como no processamento de óleos comestíveis e na indústria de cana de açúcar, onde a expansão comercial solicita melhores tecnologias. Além disso, possui vantagens, como baixo custo de operação em modo isotérmico.

Existem várias classes para a filtração tangencial, sendo que o tamanho das partículas a serem separadas é que indica qual é o melhor processo: se é a microfiltração, a ultrafiltração ou a nanofiltração. Mas o maior obstáculo inerente ao processo é o declínio do fluxo de permeado ou fluxo transmembrana que ocorre devido à concentração de polarização e à impregnação na superfície permeável (fouling). Por isso, um extenso estudo dos fenômenos de transporte é necessário para o melhor entendimento dos mecanismos de transferência de massa presentes no processo. Vários trabalhos sobre a filtração tangencial com membranas 
são encontrados na literatura com estudos analíticos, numéricos e experimentais.

O escoamento associado ao processo de filtração tangencial é descrito como um escoamento em tubo ou entre placas e vem sendo estudado com muita freqüência desde a década de 50 ([BERMAN, 1953]). Ripperger e Altamnn (2002) publicaram uma visão geral do processo de microfiltração, em que descrevem a importância desse tipo de filtração e seus mecanismos físicos presentes no processo de suspensões com pequenas partículas; além disso, discutem o desenvolvimento nos últimos anos do processo.

Trabalhos dedicados a modelos analíticos também já foram desenvolvidos: KARODE, 2001, MUNSON-McGEE, 2002, RANJAN et al., 2004a, e outros. Além dos estudos analíticos e experimentais feitos para escoamentos ao longo de paredes permeáveis, alguns pesquisadores têm apresentado modelos numéricos que procuram inserir e quantificar o fenômeno de concentração de polarização. Devido à grande importância e influência do comportamento de fluidos complexos (não-newtonianos) presentes no processamento com microfiltração, consideráveis esforços têm sido conduzidos para entender o comportamento desses fluidos em um domínio com superfície permeável.

A complexidade do problema estudado nesta tese não se restringe a um escoamento convectivo de um fluido puro. O mecanismo de transferência de massa, acentuadamente convectivo, também sofre influência de fenômenos de superfície (polarização) quando aplicado na separação de solutos. Para a mais completa descrição do problema, neste trabalho, foi implementado um modelo matemático que envolve as equações de conservação da massa, da quantidade de movimento e das espécies químicas. Em particular, investigaram-se a influência e o desempenho de esquemas de discretização dos termos convectivos das equações trabalhadas, e analisou-se o modelo para os casos de escoamento em regime laminar e turbulento de fluidos newtonianos e não-newtonianos. 


\section{Objetivos}

Reconhecendo-se o interesse considerável de soluções numéricas representativas para escoamentos incompressíveis em superfícies permeáveis associados à filtração tangencial, este trabalho tem como objetivo apresentar um modelo matemático numérico que represente esse tipo de escoamento para fluidos newtonianos e não-newtonianos com regime de escoamento laminar e estendê-lo para o regime turbulento. Para atender esta finalidade, têm-se:

- definir e implementar um modelo matemático para escoamentos de fluidos newtonianos e não-newtonianos em tubos porosos em regime laminar;

- verificar a influência e desempenho de esquemas de discretização dos termos convectivos nos resultados do escoamento abordado e definir um esquema que melhor represente os resultados do escoamento em tubos permeáveis;

- analisar a validação do modelo numérico produzido nesse trabalho a partir da comparações com a literatura e

- avaliar a extensão da modelagem implementada para o regime laminar para o escoamento em regime turbulento, utilizando-se o usual modelo de comprimento de mistura de Prandtl. 


\section{Capitulo 2}

\section{Revisão Bibliográfica}

Durante o desenvolvimento deste projeto de pesquisa, muitas definições tiveram que ser investigadas e analisadas. Para isso, detalhada pesquisa bibliográfica foi realizada com a intenção de se entender o problema estudado e de se verificar a relevância na literatura do trabalho proposto. Sendo assim, este capítulo apresenta uma revisão bibliográfica dos temas relacionados ao problema de escoamento em tubos permeáveis ou membranas tubulares.

\subsection{Conceitos básicos de filtração tangencial}

A filtração tangencial, em especial as realizadas com membranas, é um tipo de filtração que possui um grande potencial para remoção de partículas, as quais determinam, de acordo com o seu tamanho, qual é o melhor processo, se, microfiltração, ultrafiltração ou nanofiltração ([ZEMAN and ZYDNEY, 1996]). A membrana tem uma estrutura microporosa e separa partículas, de acordo com o tamanho dos poros, de um líquido ou de uma fase de gás. A separação é baseada no efeito físico de "peneira". Conseqüentemente, 
o efeito da separação limita-se na maior parte à superfície exterior da membrana. A estrutura porosa da membrana deve ter uma estreita distribuição de poros a fim de assegurar a retenção quantitativa das partículas em um dado tamanho. Uma outra característica importante é a porosidade elevada das membranas que conduz às taxas de filtração elevadas em pequenas diferenças de pressões.

Nos processos de filtração tangencial, a pressão impele somente parte do produto através da membrana, enquanto que o produto restante flui tangencialmente a superfície da membrana, como se pode observar na Fig. 2.1. A separação de partículas de materiais distintos ocorre espontaneamente em função de seus tamanhos, independentemente da temperatura e da densidade do material a separar ([RIPPERGER and ALTMANN, 2002]).

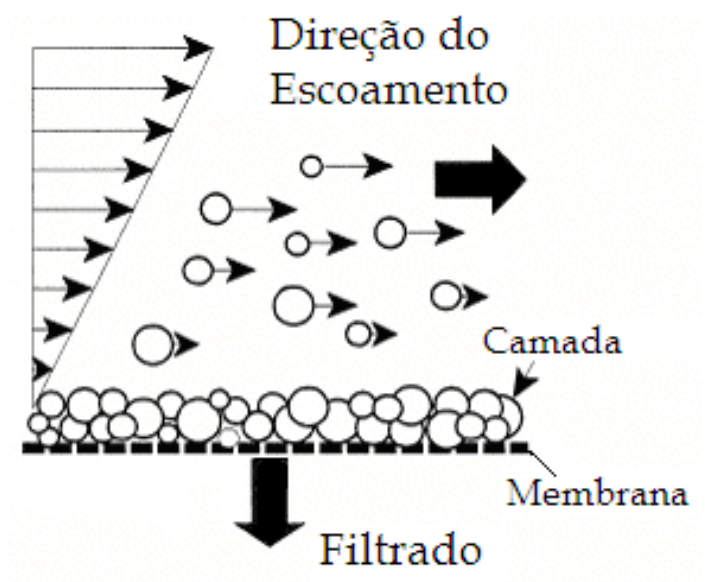

Figura 2.1: Esquema de filtração tangencial.

Durante a filtração tangencial, partículas dentro do fluxo de alimentação são convectivamente dirigidas à superfície da membrana onde elas se acumulam, enquanto que o movimento difusivo de partículas causa um transporte dessas partículas, oposto ao transporte convectivo do escoamento de filtrado (permeado). 
O acúmulo de partículas próximo à superfície da membrana é conhecido como concentração de polarização. A concentração de soluto na região adjacente à membrana varia do valor da concentração na superfície da membrana, $c_{w}$, até a concentração de alimentação, $c_{0}$, cuja distância entre esses valores é caracterizada como a espessura da camada limite de concentração, definida como $\delta$, como ilustrado a Fig. 2.2 ([ZEMAN and ZYDNEY, 1996]).

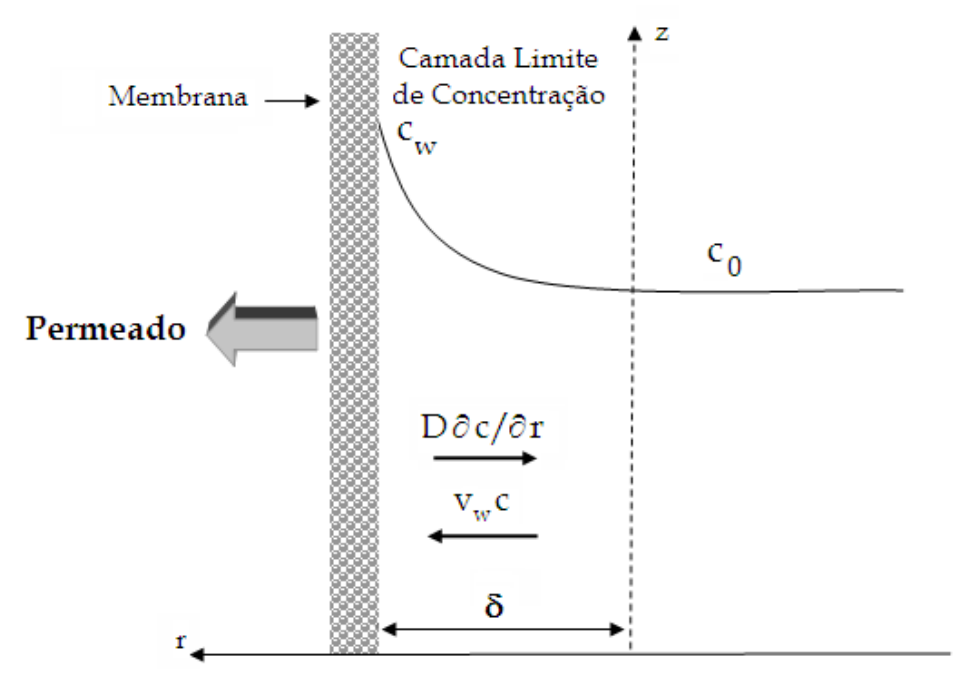

Figura 2.2: Concentração de polarização.

A camada de polarização é um fenômeno que causa uma complicação adicional ao processo de filtração, pois provoca o aumento na resistência hidráulica para o fluxo de permeado e, conseqüentemente, o declínio do fluxo com o tempo. Por isso, um melhor conhecimento da formação da camada e da deposição de partículas na membrana poderia resultar em um uso econômico da filtração tangencial em muitas aplicações técnicas ([RIPPERGER and ALTMANN, 2002]).

Lee e Clark (1997) desenvolveram um modelo numérico bidimensional para descrever o declínio do fluxo devido à camada de polarização durante a filtração tangencial e 
predizer o estado estacionário do fluxo de permeado.

Mignard e Glass (2001) apresentaram um modelo numérico para prever efeitos de variação do $\mathrm{pH}$ nos mecanismos de entupimento (fouling) próprios do processo de concentração de soluções de proteínas por microfiltração, para isso, eles utilizaram informações de modelos da literatura para a formação da camada de polarização, a fim de combiná-las com as equações de Stokes-Einsten e Bowen \& Jenner.

Yeh (2002) estudou o declínio do fluxo de permeado para ultrafiltração ao longo de membranas tubulares por meio do balanço de massa e momento, baseado na combinação do modelo de resistência em série com considerações do declínio da taxa de escoamento e pressão transmembrana ao longo do tubo. O memcionado autor concluiu que o declínio do fluxo de permeado ocorre principalmente devido ao declínio da pressão transmembrana e ao aumento da espessura da camada de concentração de polarização ao longo da membrana tubular, e portanto é, até certo ponto, sensível para pressão transmembrana menor e concentração da solução maior.

Damak et al. (2004b) apresentaram um modelo numérico para prever o mecanismo que envolve o comportamento do declínio do fluxo de permeado durante o processo de filtração tangencial. Observaram que altos valores do número de Reynolds na direção axial e do número de Schmidt conduzem a uma dimuição da espessura da camada limite de concentração.

Yeh et al. (2003) e (2004) analisaram o declínio do fluxo de permeado para a ultrafiltração por meio de um balanço das equações de momento e massa, utilizando-se do modelo de resistência em série e resultados experimentais em uma membrana do tipo fibra oca ${ }^{1}$ e em um módulo de membrana tubular.

\footnotetext{
${ }^{1}$ Hollow-fiber.
} 
Para definir o fluxo de permeado e descrever as conseqüências da concentração de polarização, a qual é responsável pela limitação do fluxo, foram desenvolvidos alguns modelos. Os principais são: modelo de gel polarizado, modelo de pressão osmótica e modelo de resitência em série.

\section{Modelo de gel polarizado}

O modelo de gel polarizado é um dos mais usados para análise do fluxo no processo de ultrafiltração ([NAKAO et al., 1979], [ZEMAN and ZYDNEY, 1996], [PARIS et al., 2002]). Baseado na teoria do filme ([ZEMAN and ZYDNEY, 1996]), o modelo pode ser usado para preverem um limite do fluxo com a suposição que ocorre na superfície permeável uma máxima concentração, chamada de concentração gel, $c_{g}$. Supõe-se, geralmente, que essa máxima concentração, $c_{g}$, reflete no ponto de gelatificação, precipitação e/ou agregação das macromoléculas na superfície da membrana ([ZEMAN and ZYDNEY, 1996]), formandose a chamada camada gel. O modelo é definido da seguinte forma:

$$
v_{w}=\kappa \ln \left(\frac{c_{g}}{c_{0}}\right)
$$

em que $v_{w}$ é a velocidade de permeação, $\kappa$ é o coeficiente de transferência de massa, $c_{g}$ é a concentração na camada gel e $c_{0}$ é a concentração de alimentação.

De acordo com esse modelo, a espessura da camada gel aumenta com a pressão, e a concentração na camada gel é constante e somente depende do soluto ([PARIS et al., 2002]). Porém, a condição de $c_{g}$ ser constante é questionável.

Nakao et al. (1979) investigaram as características da camada gel medindo diretamente a concentração na camada para soluções aquosas de álcool polivinílico e ovalbumina. Verificaram que a concentração na camada gel não é constante, mas uma função da con- 
centração e velocidade de alimentação, $c_{0}$ e $u_{0}$.

Paris et al. (2002) aplicaram o modelo em dados experimentais e verificaram que a concentração na superfície da membrana não é constante, mas é dependente da velocidade de alimentação, $u_{0}$.

Modelo de pressão osmótica

O modelo de pressão osmótica considera o limite do fluxo como conseqüencia do aumento da pressão osmótica produzida por altas concentrações de soluto rejeitado próximo à superfície da membrana, levando-se em conta que a concentração de soluto rejeitado aumente com a aplicação da pressão. Assim, pelo modelo, o fluxo de permeado pode ser expresso por:

$$
v_{w}=L_{p}(\Delta p-\Delta \Pi),
$$

em que $L_{p}$ é permeabilidade da membrana, $\Delta p$ é pressão transmembrana e $\Delta \Pi$ é a pressão osmótica de soluções macromoleculares, a qual pode ser representada pela expressão ([ZEMAN and ZYDNEY, 1996]):

$$
\Delta \Pi=A_{1} c+A_{2} c+A_{3} c
$$

em que $A_{1}, A_{2}$ e $A_{3}$ são valores constantes determinados por dados experimentais.

Paris et al. (2002) verificaram em virtude dos resultados da pressão osmótica para a solução de dextran T500, que os valores seriam insignificantes quando comparados com a pressão transmembrana. Por isso, o modelo de pressão osmótica não pode fornecer uma completa teoria para o limite do fluxo no processo de ultrafiltração, pois a pressão osmótica é desprezível em muito processos. 
De acordo com Yeh e Chen (1993b), o modelo de pressão osmótica é difícil determinar a concentração axial na superfície da membrana do tipo fibra oca ou hollow-fiber.

Todavia, esse modelo é geralmente usado por muitos autores: Wijmans et al. (1984); Wijmans et al. (1985); Yeh e Cheng (1993a); Kotzev (1994); Cheng et al. (1997); Karode (1999); Prabhakar et al. (2000); Curcio (2000); Geraldes et al. (2001); Sreenivas et al. (2001); dentre outros.

Modelo de resistência em série

O modelo de resistência em série relaciona o fluxo de permeado com a pressão transmembrana e com algumas resistências para o escoamento do solvente devido ao entupimento da membrana (fouling) e à concentração de polarização.

A lei de Darcy ([ZEMAN and ZYDNEY, 1996]) determina o fluxo transmembrana para a água pura:

$$
v_{w}=\frac{\Delta p}{R_{m}}
$$

em que $R_{m}=\frac{1}{L_{p}}$ é resistência da membrana, determinada experimentalmente por meio de medições do fluxo de permeado médio, $\bar{v}_{w}$, para várias pressões transmembrana.

Com o uso de soluções nos processos de filtração tangencial, a resistência do entupimento da membrana, $R_{f}$, e a resistência da camada de polarização e da camada limite de concentração, $R_{p}$, são adicionadas ao modelo:

$$
v_{w}=\frac{\Delta p}{R_{m}+R_{f}+R_{p}} .
$$

O parâmetro $R_{p}$ é uma função de parâmetros operacionais e propriedades físicas, portanto, ele depende da permeabilidade do gel e de sua espessura, ou seja, $R_{p}=\phi \Delta p$, 
em que $\phi$ é uma função das variáveis que afetam a transferência de massa, tais como: viscosidade, taxa de cisalhamento e temperatura ([CHERYAN, 1998]). Desta forma, o modelo de resistência em série é definido pela seguinte expressão:

$$
v_{w}=\frac{\Delta p}{R_{m}+R_{f}+\phi \Delta p}
$$

Os parâmetros $R_{f}$ e $\phi$ são obtidos utilizando-se: dados experimentais do fluxo de permeado médio, $\bar{v}_{w}$, da solução, para várias pressões transmembrana; o método linear simples para a equação da reta e a equação

$$
\frac{1}{v_{w}}=\left(R_{m}+R_{f}\right) \frac{1}{\Delta p}+\phi
$$

São muitos os trabalhos que utilizam o modelo de resistência em série para definir o fluxo de permeado.

Yeh e Cheng (1993) investigaram experimentalmente os efeitos da pressão transmembrana, velocidade do fluxo e concentração de alimentação em membranas de ultrafiltração de solução de dextran T500 em um cartucho de fibra oca. Verificaram que os dados experimentais concordam com a correlação desenvolvida, baseada no modelo de resistência em série. Concluiram também que a correlação seria apropriada a outros sistemas de ultrafiltração.

Yeh e Wu (1997) desenvolveram uma correlação para predizer o fluxo de permeado para ultrafiltração em módulos de fibra oca baseado no modelo de resistência em série. A ultrafiltração com soluções aquosas de PVP-360 foi feita em cartuchos de fibra oca e os resultados comparados com a correlação desenvolvida.

Paris et al. (2002) discutiram os modelos de gel polarizado, pressão osmótica e 
resistência em série e propuseram modificações no modelo de resistência em série para considerar a influência da concentração média de soluto na resistência devido à camada de polarização.

Além desses trabalhos podem ser encontrados na literatura muitos outros estudos que utilizam o modelo de resitência em série em suas modelagens, dentre eles, destacam-se: Yeh (2002); Moussy (2000); Yeh et al. (2004); Lee e Clark (1997); Lee e Clark (1998); Damak et al. (2004a); Damak et al. (2004b); dentre outros.

\subsubsection{Determinação da concentração na superfície da membrana}

Na filtração tangencial, a quantidade de solutos transportados para a superfície da membrana é igual à soma do fluxo de soluto que atravessa a membrana com o fluxo de soluto que retorna ao fluxo de alimentação ([ZEMAN and ZYDNEY, 1996]).

Seja um volume de controle de tamanho constante e fixo no espaço sobre uma fina camada de concentração de soluto, como pode ser observado na Fig. 2.3.

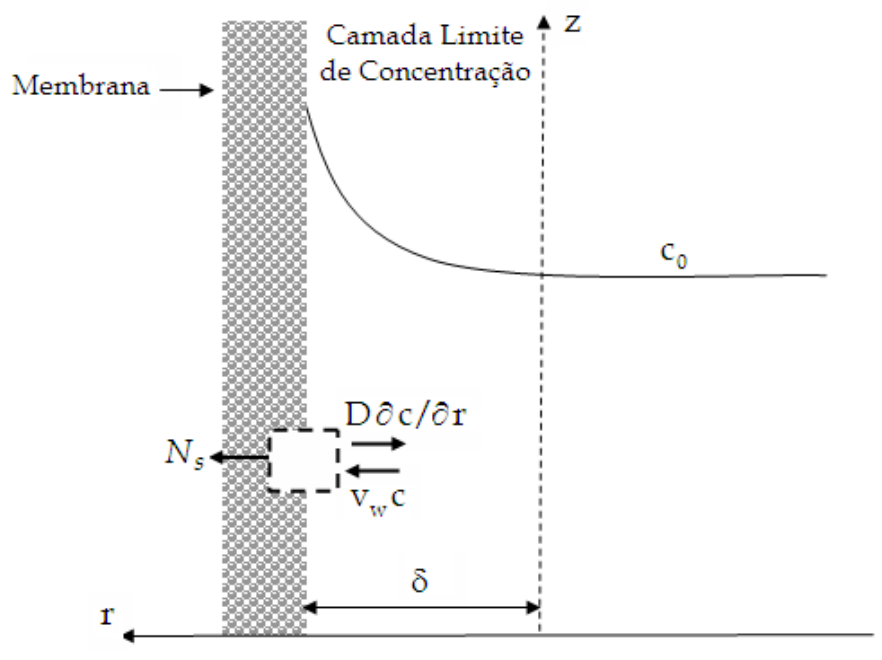

Figura 2.3: Volume de controle na camada de polarização. 
Considerando-se um sistema sem reações químicas, tem-se que o balanço de massa dentro desse volume de controle é definido da forma ( [ZEMAN and ZYDNEY, 1996]), |BIRD et al., 1990|:

$$
v_{w} c_{w}-\left.D \frac{\partial c}{\partial r}\right|_{r=R}-N_{s}=0
$$

em que $N_{s}=v_{w} c_{f}$ é o fluxo de soluto dentro da membrana e $c_{f}$ é a concentração de soluto no filtrado (permeado).

Supondo-se que $c_{f}=0$ (soluto completamente rejeitado pela membrana), tem-se a equação

$$
v_{w} c_{w}-\left.D \frac{\partial c}{\partial r}\right|_{r=R}=0
$$

Após desenvolver o termo $\frac{\partial c}{\partial r}$ da equação (2.1.9), obtém-se a concentração próxima à superfície do tubo ou membrana, $c_{w}$.

\subsubsection{Número de Sherwood}

No estudo de transferência de massa, o coeficiente de transferência de massa é um parâmetro importante para projetar os módulos de separação por membranas. Portanto, uma boa avalição desse coeficiente é necessária.

O coeficiente de transferência de massa é definido rigorosamente como a razão do fluxo difusivo do soluto na superfície do tubo ou membrana, $-\left.D(\partial c / \partial r)\right|_{r=R}$, com a concentração total na direção da força por difusão, $\left(c_{w}-c_{0}\right)$ ([ZEMAN and ZYDNEY, 1996], [CARREAU et al., 1997]): 


$$
\kappa_{\text {local }}=\frac{-\left.D \frac{\partial c}{\partial r}\right|_{r=R}}{\left(c_{w}-c_{0}\right)} .
$$

Segundo Damak et al. (2004a apud Sherwood ([SHERWOOD et al., 1975]), Sherwood analisou a transferência de massa com a intenção de encontrar uma condição semelhante entre diferentes casos de escoamentos. Para estabelecer essa similaridade, ele usou uma forma normalizada de especificar o fluxo de massa, conhecida como número de Sherwood (DAMAK et al., 2004a, CARREAU et al., 1997)

$$
S h=\frac{2 \kappa_{\text {local }} R}{D} .
$$

Substituindo-se (2.1.10) em (2.1.11), tem-se:

$$
S h=\frac{-\left.2 R \frac{\partial c}{\partial r}\right|_{r=R}}{c_{w}-c_{0}} .
$$

Em muitos casos, utiliza-se também o número de Sherwood médio, o qual é definido pela seguinte forma:

$$
\overline{S h}=\frac{1}{L} \int_{0}^{L} S h d z
$$

em que a integral é resolvida numericamente pela regra do trapézic ${ }^{2}$.

Na literatura, são poucos os trabalho relacionados com a correlação de Sherwood para escoamentos em tubos permeáveis, dentre eles, podem-se destacar: Gekas e Hallström (1987), Parvatiyar (1998), Minnikanti et al. (1999); Ranjan et al. (2004a); Ranjan et al. $(2204 b)$.

\footnotetext{
${ }^{2}$ Pode-se encontrar a regra do trapézio em livros de cálculo numérico, como por exemplo Ruggiero e Lopes (1996) e Barroso et al. (1987).
} 
Gekas e Hallström (1987) fizeram uma revisão crítica das correlações da transferência de massa para escoamento em regime turbulento. Dentro dessa revisão, realizaram uma discussão dos fatores que influenciam a transferência de massa durante operações com membranas, como porosidade e rugosidade na parede da membrana e mudança da viscosidade e coeficiente de difusão devido ao intenso gradiente de concentração. Propuseram, também, uma modificação nas equações para o número de Sherwood, em que são considerados os efeitos de rugosidade e porosidade na parede.

Parvatiyar (1998) desenvolveu modelos teóricos para descrever o fenômeno de transferência de massa em uma membrana tubular para escoamento de fluidos newtonianos e não-newtonianos. Os modelos para transferência de massa foram obtidos em virtude de considerações hidrodinâmicas do campo de escoamento. Semelhanças e vantagens desses modelos foram discutidas, e a influência de parâmetros reológicos dos fluidos na transferência de massa foi destacada.

Minnikanti et al. (1999) desenvolveram uma relação para o número de Sherwood, a fim de predizer o coeficiente de transferência de massa sobre uma camada limite de concentração desenvolvida, para regime de escoamento turbulento. A relação desenvolvida inclui os efeitos de sucção através da membrana para a ultrafiltração, e é usada em união com o modelo de pressão osmótica para predizer o fluxo de permeado em ultrafiltração governada por pressão osmótica. Os resultados foram comparados com dados experimentais obtidos da literatura.

Ranjan et al. (2004a) obtiveram uma expressão para o número de Sherwood considerando fluidos não-newtonianos (pseudoplásticos e dilatantes) em regime de escoamento laminar em módulos de membranas. O efeito de sucção é incorporado ao coeficiente de transferência de massa de uma teoria básica. Os efeitos das condições de operação no 
coeficiente de transferência de massa são investigados.

Ranjan et al. (2004b) obtiveram uma expressão para o número de Sherwood para fluidos não-newtonianos (pseudoplásticos e dilatantes) em regime de escoamento turbulento com sucção. As soluções são obtidas por membranas de configurações comuns, ou seja, canal retangular e módulos tubulares.

\section{$2.2 \quad$ Reologia}

A reologia é o estudo do escoamento e deformação da matéria, ou seja, a reologia é o estudo do comportamento de fluidez. Os componentes dos fluidos podem apresentar diferentes formas geométricas, características diversas de ligação química, tamanhos variados, que lhes conferem comportamentos distintos. Assim, devido a sua composição, alguns produtos possuem uma única viscosidade a uma dada temperatura, independente da força de cisalhamento, e são denominados de fluidos Newtonianos, enquanto outra grande parte dos fluidos apresentam comportamento reológico mais complexo e a determinação da viscosidade não é um tópico simples ([MACHADO, 2000]).

A relação entre a tensão de cisalhamento e a taxa de deformação define, de certo modo, o comportamento reológico dos líquidos considerados puramente viscosos. A Fig.2.4 apresenta curvas características para alguns tipos de fluidos, ela é um registro gráfico que mostra como a tensão de cisalhamento varia em função da taxa de cisalhamento.

Os fluidos viscosos, portanto, podem ser classificados em função do seu comportamento reológico. Este envolve a determinação experimental e a análise da relação entre a tensão cisalhante e o gradiente de velocidade ou taxa de deformação, para uma determinada condição de temperatura e pressão. 
A curva de fluxo mais simples é a de uma reta interceptando o encontro dos eixos cartesianos, que define o comportamento dos fluidos simples, cujo estudo foi desenvolvido por Newton. Na Fig,2.4, a curva de fluido newtoniano refere-se a este comportamento. Todas as curvas da figura, exceto a curva que indica fluido newtoniano, se referem a tipos ou a modelos de fluidos não-newtonianos.

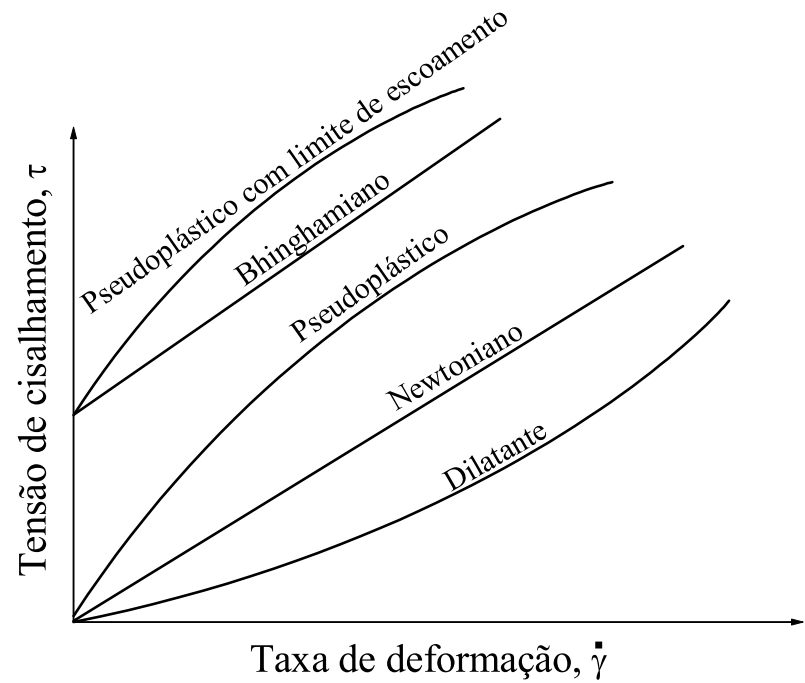

Figura 2.4: Esquema de classificação dos fluidos, segundo comportamento reológico.

\section{Fluidos Newtonianos}

O fluido newtoniano é aquele cuja viscosidade é independente da taxa de deformação a que ele está submetido. Possui um único valor de viscosidade, em uma dada temperatura. Exemplos: gases, água, leite, soluções de sacarose, óleos vegetais, fluidos de silicone, solventes e soluções muito diluídas.

Fluidos Não-Newtonianos

O fluido não-newtoniano possui a relação entre taxa de deformação e tensão de 
cisalhamento mais complexa, uma vez que a viscosidade varia com a taxa de deformação e com a tensão de cisalhamento inicial. Os fluidos não-newtonianos são classificados como aqueles que:

- possuem propriedades reológicas que não dependem do tempo de aplicação da tensão de cisalhamento como os pseudoplásticos (polpas de frutas, caldos de fermentação, melaço de cana, tintas, emulsões e gomas) e dilatantes (soluções de amido e soluções de farinha de milho e açúcar), os quais não possuem tensão de cisalhamento inicial, e plásticos de Bingham e Herschel-Bulkley que possuem tensão de cisalhamento inicial;

- possuem propriedades reológicas que dependem do tempo de aplicação da tensão de cisalhamento, como tixotrópicos e reopéticos e

- são substâncias que apresentam propriedades viscosas e elásticas acopladas, e em cujo término da tensão de cisalhamento ocorre uma certa recuperação da deformação. Exemplos: massas de farinha de trigo, gelatinas, queijos.

\subsection{Equação reológica de estado}

Para uma ampla classe de materiais, as componentes do tensor das tensões viscosas, $\tau_{i, j}$, dependem somente das componentes do tensor taxa de deformação ([BREDA and D'AVILA, 2000]), $\overline{\dot{\gamma}}_{i, j}$. Assim, chama-se equação reológica de estado ou equação constitutiva a relação entre estas duas variáveis. Para determinar o campo de fluxo de um material, ou seja, conhecer $\vec{u}, \overrightarrow{\nabla p}, \mathrm{~T}$ e c, é necessário solucionar simultaneamente as equações de conservação e as equações reológicas de estado.

Para o escoamento de fluidos não-newtonianos pseudoplásticos, muito utilizados em 
diversas aplicações industrias do processo de filtração tangencial, a equação reológica de estado usada é

$$
\tau_{i, j}=\eta \overline{\dot{\gamma}}_{i, j}
$$

em que $\overline{\dot{\gamma}}$ é o tensor taxa de deformação e $\eta$ é a viscosidade aparente, a qual pode ser representada pela lei das potências ${ }^{3}$, definida pela seguinte relação (|CARREAU et al., 1997|):

$$
\eta=m \dot{\gamma}^{n-1}
$$

em que $m$ é o coeficiente de consistência $\left(P a \cdot s^{n}\right), \dot{\gamma}$ é a taxa de deformação e $n$ é o índice de comportamento.

A mencionada equação (2.3.2) fica equivalente à de fluido newtoniano quando $n=1$, quando $n>1$; o material apresenta comportamento dilatante e, quando $n<1$, o fluido apresenta comportamento pseudoplástico ([BRETAS and D'AVILA, 2000]).

\subsection{Modelagem}

Em soluções numéricas, a modelagem matemática é estruturada por um conjunto de equações de conservação que são resolvidas em um computador, o qual produzirá resultados do problema em questão. Muitos tipos de escoamentos de fluidos podem ser estudados por meio da modelagem matemática, em especial, o escoamento em tubos com paredes permeáveis e/ou em membranas tubulares. Na literatura, pode-se encontrar vários trabalhos que estudaram esse tipo escoamento e seus fenômenos por meio de modelagem

\footnotetext{
${ }^{3}$ Conhecida também como power-law model.
} 
matemática numérica ou analítica.

Hung e Perng (1991) investigaram o desenvolvimento hidrodinâmico de um fluido não-newtoniano em um tubo circular com paredes porosas para o caso de injeção e sucção constante, em regime de escoamento laminar. A fim de obterem tal resultado, os autores resolveram as equações de conservação de massa e de quantidade de movimento, generalizadas para fluidos não-newtoniano, utilizando-se de diferenças finitas e o método SIMPLER para o cálculo dos campos de pressão e velocidades. Como resultados, distribuição de velocidade, queda de pressão e coeficiente de atrito são apresentados para vários índices de comportamento. Vale ressaltar que apenas os resultados com fluidos newtonianos são comparados com resultados da literatura.

Kotzev (1994) apresentou um tratamento numérico para o processo de ultrafiltração de uma solução fluindo em um módulo de membrana tubular, para altos números de Reynolds. Utilizou o esquema de diferenças finitas em malha uniforme, esquema de discretização upwind e diferenças centrais para resolver numericamente as equações de conservação de massa, de quantidade de movimento e conservação das espécies, para fluidos newtonianos e regime de escoamento laminar. Resultados representando a concentração na membrana, velocidade de permeação e fração do solvendo removida são apresentados. Em que pese os resultados não terem sido comparados com dados experimentais, o citado autor verificou que aqueles concordam com a tendência geral da ultrafiltração.

Nassehi (1998) teve como principal objetivo o desenvolvimento de um esquema para a imposição de um escoamento isotérmico, laminar, não-newtoniano em um domínio anular com paredes permeáveis. Para isso, utilizou o acoplamento das equações de conservação de massa e de quantidade de movimento com a equação de Darcy, para determinar o fluxo de permeado, e o método de elementos finitos para resolver numericamente as 
equações de conservação.

Lee e Clark (1998) desenvolveram um modelo numérico simples para descrever o declínio do fluxo de permeado durante o processo de ultrafiltração de suspensões de colóides, em regime de escoamento laminar. A fim de estudar tal fenômeno, utilizaram a equação parabólica de conservação das espécies químicas e o modelo de resistência em série. De acordo com os autores, o modelo mostrou-se uma ferramenta de ajuda para investigar o efeito de vários parâmetros de operação, tais como: tamanho da partícula, concentração de alimentação, velocidade axial e dimensões da membrana. As simulações mostraram que o modelo produziu resultados com boa concordância com resultados experimentais.

Geraldes et al. (2001) estudaram um modelo matemático baseado na formulação de volumes finitos para predizer a hidrodinâmica do escoamento laminar e a transferência de massa de soluções aquosas em um canal com sistemas de placas, junto com condições de fronteira, as as quais levam em conta o transporte de soluto no interior da membrana. Implementaram as equações de conservação de massa, conservação da quantidade de movimento e conservação das espécies químicas, em duas dimensões. Os termos convectivos foram discretizados por um esquema híbrido (diferenças centrais/upwind) e o método SIMPLE foi utilizado para calcular os campos de pressão e velocidades. Propuseram um fator de correção, dependente do soluto da concentração na superfície da membrana, para o modelo de pressão osmótica e uma correlação para a espessura da concentração na camada limite.

Karode (2001) desenvolveu uma solução analítica para a queda de pressão em escoamento em canal retangular e em tubo cilíndrico para paredes porosas, considerando-se a permeabilidade da membrana ou do tubo permeável como constante. O trabalho conduziu a uma expressão analítica para a queda de pressão em função da permeabilidade da 
parede, da dimensão do canal, da posição axial e das propriedades do fluido. Segundo o autor, essa expressão analítica para permeabilidade constante poderia ser usada como uma benchmark solution para escoamentos de fluidos por membranas semi-permeáveis.

Bowen e Williams (2001) investigaram a influência das condições físico-químicas na taxa de permeação em processos de ultrafiltração de suspensões de colóides, para regime de escoamento laminar. Com o fim de atender a esta necessidade, implementaram em FORTRAN um modelo matemático formado pela equação de conservação de massa, e pelas equações parabólicas de conservação da quantidade de movimento e conservação das espécies. O modelo considerou a variação dos efeitos de pressão osmótica, coeficiente de difusão e viscosidade. O fluxo de permeado é determinado pelo modelo de pressão osmótica, e as equações são resolvidas utilizando diferenças finitas e o algoritmo de Thomas. Os resultados do modelo foram comparados com dados experimentais e observou-se uma boa concordância entre os resultados.

Richardson e Nassehi (2003) estudaram um modelo numérico que simula o campo de concentração ao longo de uma parede permeável, para escoamento de fluidos nãonewtonianos. O algoritmo é baseado no esquema de elementos finitos Petrov-Galerkin (SUPG) para a solução das equações de conservação de massa, da quantidade de movimento e da conservação das espécies, em domínio de duas dimensões e em coordenadas cartesianas. Esse modelo considera as variações de alguns parâmetros, os quais ocorrem quando parte do fluido se infiltra na parede porosa do domínio. Segundo os mencionados autores, esse tipo de escoamento é um sistema de duas fases, em que o comportamento dinâmico do fluido é influenciado pelas interações entre partículas sólidas e o transporte de fluido, mas que devido à falta de técnicas práticas para medição de coeficientes necessários na descrição da força de arrasto e no efeito da interação das partículas, não é possível 
a inclusão deste tipo de fenômeno em um modelo desenvolvido para fins de engenharia, assim, consideram o fluido e as partículas movendo-se com a mesma velocidade.

Damak et al. (2004a) apresentaram uma investigação teórica do depósito de partículas sobre a superfície permeável em membranas tubulares. A expressão matemática, para o estado estacionário e escoamento de fluido newtoniano de regime laminar, é formada pelas equações de conservação de massa, da quantidade de movimento e das espécies químicas em duas dimensões. Utilizaram diferenças finitas e apresentaram um esquema numérico para a resolução dessas equações. Investigaram no escoamento os efeitos do número de Reynolds, do número de Reynolds na parede e do número de Schmidt. Apresentaram correlações para a espessura da camada limite de concentração e do número de Sherwood, baseadas em valores do campo de concentração nas simulações feitas pelo modelo.

Damak et al. (2004c) descreveram um modelo para simular processos de filtração tangencial em membranas tubulares, para regime de escoamento laminar de fluidos newtonianos. A sucção variável na parede permeável é descrita pela lei de Darcy. O fluxo de alimentação no tubo, considerado desenvolvido e tangente à parede porosa, é modelado pelas equações de conservação de massa e de quantidade de movimento. Várias simulações para diferentes números de Reynolds axiais e Reynolds na parede são apresentadas. Os resultados são discutidos com a intensão de estudar a validade do problema.

Bacchin et al. (2005) discutiram, por meio de modelo analítico e por experimentos, as conseqüências do fluxo crítico na filtração tangencial. Segundo os autores, o fluxo crítico é um conceito que descreve o menor fluxo de permeado no qual o efeito de entupimento (fouling) aparece na membrana. Os resultados teóricos mostraram boa concordância com os dados experimentais da filtração tangencial de suspensões de latex e BSA.

Venezuela et al. (2007) utilizaram a técnica da transformada integral generalizada 
(GITT) para obter a solução do escoamento em regime laminar e permanente em tubos permeáveis de um fluido newtoniano e incompressível. A formulação matemática utilizou-se da equação diferencial parabólica da conservação das espécies químicas (equação convectiva-difusiva). Os perfis de velocidade para o escoamento de região de entrada, que estão presentes nos termos convectivos da equação, foram avaliados com soluções obtidas na literatura. A velocidade na parede permeável foi considerada uniforme, sendo a concentração na parede do tubo variável com a posição axial. Uma funcional expressão matemática para a espessura da camada limite de concentração polarizada foi proposta nesse trabalho de acordo com a técnica da transformada integral. Uma metodologia computacional com análise de erro foi utilizada para determinar o perfil de concentração na parede e da espessura da camada de concentração polarizada. Os resultados obtidos para o fluxo transmembrana local e para a espessura da camada limite de concentração polarizada foram comparados com outros da literatura.

Além dos trabalhos comentados, pode-se citar outros estudos que apresentam modelos para o escoamento de fluidos em tubos permeáveis, tais como: [SCHMITZ et al., 1992]; BASHIR and REUSS, 1992; SETHI and WIESNER, 1997; RAHIMI et al., 2005; BOWEN and WILLIANS, 2001; dentre outros.

Uma observação importante a ser considerada é o fato que, dentre os trabalhos encontrados que estão relacionados aos modelos numéricos para escoamento em tubos porosos, nenhum descreve o escoamento de fluidos não-newtonianos em regime turbulento. 


\section{Descrição da Modelagem Matemática}

Conforme exclarecido no capítulo 1, este trabalho tem por objetivos realizar um estudo numérico do escoamento de fluidos newtonianos e não-netonianos em tubos permeáveis ou em membranas tubulares para regime de escoamento laminar, e estender o estudo para regime de escoamento turbulento, com o modelo de comprimento de mistura de Prandtl. Sendo assim, apresenta-se neste capítulo uma descrição do modelo matemático implementado e analisado nesta pesquisa.

\subsection{Descrição do domínio}

Considerando um escoamento incompressível, com simetria radial, isotérmico e nãodesenvolvido em um tubo cilíndrico com paredes porosas. Como se trata de um escoamento com simetria radial, apenas metade do tubo é discretizada $(r=0$ à $r=\mathrm{R})$, onde o sistema de coordenadas tem origem na entrada, o eixo $z$ está na linha central, o eixo $r$ é normal à linha central, o raio do tubo é R e o comprimento é L, como ilustrado na Fig. 3.1. 


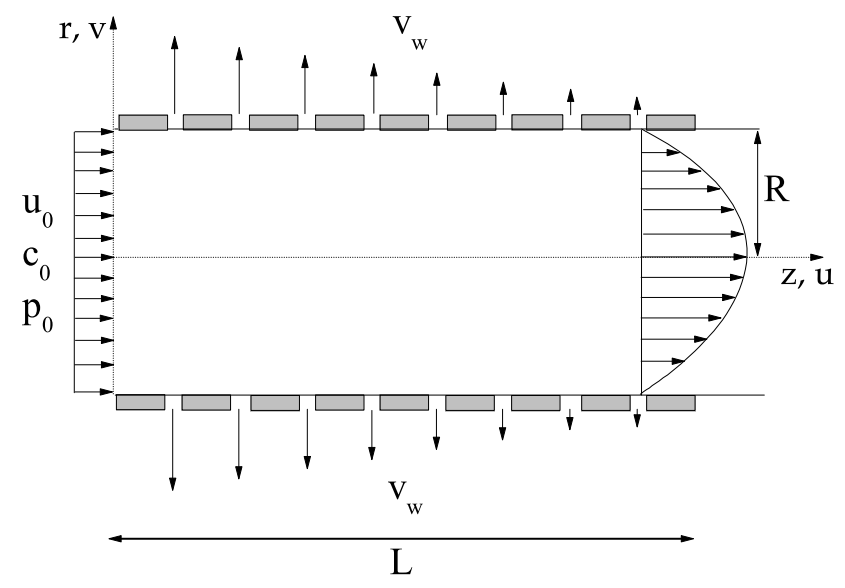

Figura 3.1: Representação do domínio do problema.

\subsection{Formulação matemática do escoamento laminar}

Escoamentos laminares são aqueles cujas camadas muito finas ou lâminas de fluidos parecem deslizar umas sobre as outras ([FORTUNA, 2000]). Assim, o escoamento viscoso, incompressível, isotérmico e laminar é descrito pelas equações de conservação de massa e de quantidade de movimento, que junto com a equação de conservação das espécies químicas constituem um sistema de equações diferenciais parciais que descrevem o escoamento do problema estudado no presente trabalho.

Conservação de massa

$$
\nabla \cdot \mathbf{u}=0
$$

Conservação da quantidade de movimento

$$
\rho \frac{D \mathbf{u}}{D t}=\nabla \cdot \sigma+\rho \mathbf{g}
$$


Conservação das espécies químicas

$$
\frac{D c}{D t}=D \nabla^{2} c,
$$

em que $\mathbf{u}=(u, v), \rho, \sigma, \mathbf{g}$ e $D$ são respectivamente, o vetor velocidade do fluido, massa específica, tensor das tensões, vetor força da gravidade - o qual não terá influência no escoamento e por isso será desprezado -, e o coeficiente de difusividade (considerado constante).

Para esse problema as equações constitutivas são dadas por:

$$
\sigma=-p \mathbf{I}+\tau, \quad \tau=2 \eta(\dot{\gamma}) \overline{\dot{\gamma}}, \quad \overline{\dot{\gamma}}=\frac{1}{2}\left(\nabla \mathbf{u}+(\nabla \mathbf{u})^{T}\right), \quad \dot{\gamma}=\sqrt{2 \operatorname{tr}(\overline{\dot{\gamma}})}
$$

em que $p$ é a pressão do fluido, I é o tensor identidade, $\tau$ é o tensor das tensões viscosas, $\overline{\dot{\gamma}}$ é o tensor taxa de deformação, $\dot{\gamma}$ é a taxa de cisalhamento (ou taxa de deformação) e $\eta(\dot{\gamma})$ é a viscosidade aparente, a qual é definida pela lei de potências (equação $(\overline{2.3 .2})$ ) e que pode ser reescrita como:

$$
\eta=m\left\{2\left[\left(\frac{\partial u}{\partial x}\right)^{2}+\left(\frac{\partial v}{\partial r}\right)^{2}+\left(\frac{v}{r}\right)^{2}\right]+\left(\frac{\partial u}{\partial r}+\frac{\partial v}{\partial z}\right)^{2}\right\}^{\frac{n-1}{2}}
$$

Assim, a partir de (2.3.2) e (3.2.4), as equações (3.2.1), (3.2.2) e (3.2.3) podem ser reescritas como:

$$
\begin{gathered}
\frac{\partial u}{\partial z}+\frac{1}{r} \frac{\partial r v}{\partial r}=0 \\
\frac{\partial u}{\partial t}+u \frac{\partial u}{\partial z}+\frac{v}{r} \frac{\partial(r u)}{\partial r}=-\frac{1}{\rho} \frac{\partial p}{\partial z}+\frac{1}{\rho} \frac{\partial}{\partial z}\left(2 \eta \frac{\partial u}{\partial z}\right)+\frac{\eta}{\rho r}\left(\frac{\partial u}{\partial r}+\frac{\partial v}{\partial z}\right)+\frac{1}{\rho} \frac{\partial}{\partial r}\left[\eta\left(\frac{\partial u}{\partial r}+\frac{\partial v}{\partial z}\right)\right] \\
\frac{\partial v}{\partial t}+u \frac{\partial v}{\partial z}+\frac{v}{r} \frac{\partial(r v)}{\partial r}=-\frac{1}{\rho} \frac{\partial p}{\partial r}+\frac{1}{\rho} \frac{\partial}{\partial r}\left(2 \eta \frac{\partial v}{\partial r}\right)+\frac{2 \eta}{\rho r} \frac{\partial v}{\partial r}-\frac{2 \eta}{\rho} \frac{v}{r^{2}}+\frac{1}{\rho} \frac{\partial}{\partial z}\left[\eta\left(\frac{\partial u}{\partial r}+\frac{\partial v}{\partial z}\right)\right],
\end{gathered}
$$




$$
\frac{\partial c}{\partial t}+u \frac{\partial c}{\partial z}+\frac{v}{r} \frac{\partial(r c)}{\partial r}=D\left(\frac{\partial^{2} c}{\partial r^{2}}+\frac{\partial^{2} c}{\partial z^{2}}+\frac{1}{r} \frac{\partial c}{\partial r}\right)
$$

As equações (3.2.7), (3.2.8) e (3.2.9) estão na forma não-conservativas; para transformá-las na forma conservativa é necessário adicionar às respectivas equações os seguintes termos: $u\left(\frac{\partial u}{\partial z}+\frac{1}{r} \frac{\partial r v}{\partial r}\right), v\left(\frac{\partial u}{\partial z}+\frac{1}{r} \frac{\partial r v}{\partial r}\right)$ e $c\left(\frac{\partial u}{\partial z}+\frac{1}{r} \frac{\partial r v}{\partial r}\right)$ ([FORTUNA, 2000]). Dessa forma, tem-se:

$$
\begin{gathered}
\frac{\partial u}{\partial t}+\frac{\partial(u u)}{\partial z}+\frac{1}{r} \frac{\partial(r v u)}{\partial r}=-\frac{1}{\rho} \frac{\partial p}{\partial z}+\frac{1}{\rho} \frac{\partial}{\partial z}\left(2 \eta \frac{\partial u}{\partial z}\right)+\frac{\eta}{\rho r}\left(\frac{\partial u}{\partial r}+\frac{\partial v}{\partial z}\right)+\frac{1}{\rho} \frac{\partial}{\partial r}\left[\eta\left(\frac{\partial u}{\partial r}+\frac{\partial v}{\partial z}\right)\right], \\
\frac{\partial v}{\partial t}+\frac{\partial(u v)}{\partial z}+\frac{1}{r} \frac{\partial(r v v)}{\partial r}=-\frac{1}{\rho} \frac{\partial p}{\partial r}+\frac{1}{\rho} \frac{\partial}{\partial r}\left(2 \eta \frac{\partial v}{\partial r}\right)+\frac{2 \eta}{\rho r} \frac{\partial v}{\partial r}-\frac{2 \eta}{\rho} \frac{v}{r^{2}}+\frac{1}{\rho} \frac{\partial}{\partial z}\left[\eta\left(\frac{\partial u}{\partial r}+\frac{\partial v}{\partial z}\right)\right], \\
\frac{\partial c}{\partial t}+\frac{\partial(u c)}{\partial z}+\frac{1}{r} \frac{\partial(r v c)}{\partial r}=D\left(\frac{\partial^{2} c}{\partial r^{2}}+\frac{\partial^{2} c}{\partial z^{2}}+\frac{1}{r} \frac{\partial c}{\partial r}\right),
\end{gathered}
$$

Portanto, a formulação matemática para o regime de escoamento laminar é formada pelas equações $(3.2 .6),(3.2 .10),(3.2 .11)$ e (3.2.12).

\subsubsection{Formulação matemática do escoamento turbulento}

Além do regime laminar, implementaram-se também as equações de conservação para o escoamento em regime turbulento.

São numerosas as formas de se modelar e simular os efeitos da turbulência. Elas variam desde as correlações e diagramas empíricos até as modernas metodologias de simulações numéricas, destacando-se dessas últimas: DNS (Direct Numerical Simulation), LES (Large-Eddy Simulation) e RANS (Reynolds-Averaged Navier-Stokes). Neste trabalho de pesquisa, utilizou-se a metodologia RANS ([FERREIRA, 2001, FREIRE et al., 2002]), 
em que a média temporal de Reynolds, um caso particular de filtro, é utilizada para simplificar a modelagem "submalha", ou seja, informações sobre flutuações turbulentas são obtidas do tensor de tensões de Reynolds.

\section{Equações média de Reynolds (RANS)}

A técnica de passagem da média de Reynolds é constituida de dois passos ( FREIRE et al., 2002 ).

1. As variáveis que aparecem nas equações de conservação são decompostas em partes médias e flutuantes (decomposição de Reynolds)

$$
\Phi=\bar{\Phi}+\Phi^{\prime}
$$

em que $\Phi$ é uma quantidade instantânea qualquer e $\Phi^{\prime}$ é flutuação instantânea.

2. É aplicado o operador média temporal sobre um intervalo de tempo finito nos termos resultantes:

$$
\bar{\Phi}=\frac{1}{T} \int_{0}^{T} \Phi(\mathbf{x}, t) d t
$$

Para que a média, definida na equação (3.2.14), possa ser usada em operações sobre as equações de movimento e de transferência de propriedades físicas em fluidos, têm-se as seguintes propriedades ${ }^{1}$ :

$$
\begin{gathered}
\overline{g+h}=\bar{g}+\bar{h} \\
\overline{a g}=a \bar{g}, \quad \text { a uma constante, }
\end{gathered}
$$

\footnotetext{
${ }^{1}$ Maiores detalhes ver FREIRE et al., 2002, SCHULZ, 2003, ROMA, 2003.
} 


$$
\begin{aligned}
\overline{\bar{g} h} & =\bar{g} \bar{h}, \\
\overline{\bar{g}} & =\bar{g}, \\
\overline{\bar{g}} \bar{h} & =\bar{g} \bar{h}, \\
\overline{\bar{g} g^{\prime}} & =\bar{g} \overline{g^{\prime}}=0, \\
\overline{g^{\prime}} & =0, \\
\overline{\frac{\partial g}{\partial s}}=\frac{\partial \bar{g}}{\partial s}, \quad \text { s é uma variável independente. } &
\end{aligned}
$$

Assim, pode-se deduzir as equações (3.2.6), (3.2.10), (3.2.11) e (3.2.12) para um escoamento de regime turbulento. Admitindo-se que as variáveis, $u, v, p, \eta$ e $c$ sejam variáveis instantâneas, tem-se:

$$
u=\bar{u}+u^{\prime} ; \quad v=\bar{v}+v^{\prime} ; \quad p=\bar{p}+p^{\prime} ; \quad \eta=\bar{\eta}+\eta^{\prime} ; \quad c=\bar{c}+c^{\prime} ;
$$

em que $u^{\prime}$ e $v^{\prime}$ são as componentes de flutuação instantânea de velocidades e $p^{\prime}, \eta^{\prime}$ e $c^{\prime}$ são as componentes de flutuação instantânea de pressão, viscosidade aparente e concentração, respectivamente. Substituindo-se as variáveis instantâneas pela decomposição (3.2.23), obtém-se a formulação matemática para o escoamento em regime turbulento.

Conservação de massa

$$
\frac{\partial\left(\bar{u}+u^{\prime}\right)}{\partial z}+\frac{1}{r} \frac{\partial\left(r\left(\bar{v}+v^{\prime}\right)\right)}{\partial r}=0
$$

Aplicando-se o operador média sobre a equação 3.2.24, obtém-se:

$$
\overline{\frac{\partial\left(\bar{u}+u^{\prime}\right)}{\partial z}+\frac{1}{r} \frac{\partial\left(r\left(\bar{v}+v^{\prime}\right)\right)}{\partial r}}=0 .
$$


Utilizando-se as equações (3.2.15), (3.2.18) e (3.2.22) em (3.2.25), tem-se:

$$
\frac{\partial \bar{u}}{\partial z}+\frac{\partial \bar{u}^{\prime}}{\partial z}+\frac{1}{r} \frac{\partial r \bar{v}}{\partial r}+\frac{1}{r} \frac{\partial r \bar{v}^{\prime}}{\partial r}=0
$$

Como a média das flutuações é igual a zero (equação (3.2.21)):

$$
\frac{\partial \overline{u^{\prime}}}{\partial z}+\frac{1}{r} \frac{\partial r \overline{v^{\prime}}}{\partial r}=0
$$

resulta

$$
\frac{\partial \bar{u}}{\partial z}+\frac{1}{r} \frac{\partial r \bar{v}}{\partial r}=0
$$

e, finalmente, da equação (3.2.24):

$$
\frac{\partial u^{\prime}}{\partial z}+\frac{1}{r} \frac{\partial r v^{\prime}}{\partial r}=0
$$

Conclui-se que a equação de conservação de massa é válida para o escoamento turbulento, considerando-se tanto a velocidade instantânea quanto as pertubações ([ROMA, 2003]).

Conservação da quantidade de movimento na direção z

Os procedimentos seguidos para a equação de conservação de massa podem ser igualmente utilizados para obter a evolução da velocidade média em escoamentos turbulentos ([SCHULZ, 2003] $)$, a partir das equações (3.2.10) e (3.2.11). Como o desenvolvimento das equações de conservação da quantidade de movimento para o regime turbulento é similar, apresenta-se apenas a dedução da componente $z$.

Considera-se a equação (3.2.10), efetua-se uma decomposição das variáveis envolvi- 
das em uma componente média e em uma componente de flutuação e aplica-se o operador média:

$$
\begin{gathered}
\overline{\frac{\partial\left(\bar{u}+u^{\prime}\right)}{\partial t}+\frac{\partial\left(\bar{u}+u^{\prime}\right)^{2}}{\partial z}+\frac{1}{r} \frac{\partial\left(r\left(\bar{v}+v^{\prime}\right)\left(\bar{u}+u^{\prime}\right)\right)}{\partial r}}=\overline{\frac{1}{\rho} \frac{\partial\left(\bar{p}+p^{\prime}\right)}{\partial z}+} \\
\frac{\frac{1}{\rho} \frac{\partial}{\partial z}\left(2\left(\bar{\eta}+\eta^{\prime}\right) \frac{\partial\left(\bar{u}+u^{\prime}\right)}{\partial z}\right)+\frac{\left(\bar{\eta}+\eta^{\prime}\right)}{\rho r}\left(\frac{\partial\left(\bar{u}+u^{\prime}\right)}{\partial r}+\frac{\partial\left(\bar{v}+v^{\prime}\right)}{\partial z}\right)+}{} \\
\frac{\frac{1}{\rho} \frac{\partial}{\partial r}\left[\left(\bar{\eta}+\eta^{\prime}\right)\left(\frac{\partial\left(\bar{u}+u^{\prime}\right)}{\partial r}+\frac{\partial\left(\bar{v}+v^{\prime}\right)}{\partial z}\right)\right]}{}
\end{gathered}
$$

Utilizando-se as propriedades (3.2.15), (3.2.18) e (3.2.21), tem-se:

$$
\begin{aligned}
& \frac{\partial \bar{u}}{\partial t}+\frac{\partial(\bar{u})^{2}}{\partial z}+\frac{\partial\left(\bar{u}^{\prime}\right)^{2}}{\partial z}+\frac{1}{r} \frac{\partial(r \overline{v u})}{\partial r}+\frac{1}{r} \frac{\partial\left(r \overline{v^{\prime} u^{\prime}}\right)}{\partial r}=-\frac{1}{\rho} \frac{\partial \bar{p}}{\partial z}+ \\
& +\frac{1}{\rho} \frac{\partial}{\partial z}\left(2 \bar{\eta} \frac{\partial \bar{u}}{\partial z}\right)+\frac{\bar{\eta}}{\rho r}\left(\frac{\partial \bar{u}}{\partial r}+\frac{\partial \bar{v}}{\partial z}\right)+\frac{1}{\rho} \frac{\partial}{\partial r}\left[\bar{\eta}\left(\frac{\partial \bar{u}}{\partial r}+\frac{\partial \bar{v}}{\partial z}\right)\right],
\end{aligned}
$$

em que,

$$
\bar{\eta}=m\left\{2\left[\left(\frac{\partial \bar{u}}{\partial x}\right)^{2}+\left(\frac{\partial \bar{v}}{\partial r}\right)^{2}+\left(\frac{\bar{v}}{r}\right)^{2}\right]+\left(\frac{\partial \bar{u}}{\partial r}+\frac{\partial \bar{v}}{\partial z}\right)^{2}\right\}^{\frac{n-1}{2}}
$$

Rearranjando-se os termos da equação (3.2.31), encontra-se:

$$
\begin{gathered}
\frac{\partial \bar{u}}{\partial t}+\frac{\partial(\bar{u})^{2}}{\partial z}+\frac{1}{r} \frac{\partial(r \bar{v} \bar{v})}{\partial r}=-\frac{1}{\rho} \frac{\partial \bar{p}}{\partial z}+\frac{1}{\rho} \frac{\partial}{\partial z}\left(2 \bar{\eta} \frac{\partial \bar{u}}{\partial z}-\left(\overline{u^{\prime}}\right)^{2}\right)+\frac{1}{\rho r}\left[\bar{\eta}\left(\frac{\partial \bar{u}}{\partial r}+\frac{\partial \bar{v}}{\partial z}\right)-\overline{v^{\prime} u^{\prime}}\right]+ \\
+\frac{1}{\rho} \frac{\partial}{\partial r}\left[\bar{\eta}\left(\frac{\partial \bar{u}}{\partial r}+\frac{\partial \bar{v}}{\partial z}\right)-\overline{v^{\prime} u^{\prime}}\right]
\end{gathered}
$$

Observa-se que a equação (3.2.33) difere da equação (3.2.10) apenas pela presença da média do produto das flutuações de velocidade, $\overline{u^{\prime} v^{\prime}}$ e $\overline{u^{\prime}}$, o chamado tensor de Reynolds 
$\left(\tau_{z r}^{t}\right.$ e $\left.\tau_{z z}^{t}\right)$. Esses termos representam a transferência de quantidade de movimento adicional, causada pela turbulência. Como tais tensões são adicionais ao termo viscoso da equação e possuem influência semelhante sobre o escoamento, elas são chamadas de tensões turbulentas. As variáveis independentes para o caso turbulento passam, portanto, a ser $\bar{u}, \bar{v}, \bar{p}, \bar{u} \bar{v}, \bar{u} \bar{u}$ e $\bar{v} \bar{v}$. Isso faz com que o número de incógnitas seja superior ao número de equações disponíveis. Para resolver qualquer problema que envolva um escoamento turbulento é, então, necessário providenciar um novo conjunto de equações que relacione as grandezas médias às grandezas instantâneas. Esse procedimento de obtenção de novas relações funcionais que completam a formulação do problema é chamado, em turbulência, de "problema de fechamento".

O modo mais simples de se definir o problema é considerar que os fenômenos de transferência de quantidade de movimento molecular e turbulento se processem de modo análogo. Essa abordagem proposta por Boussinesq (1877) sugere que a tensão de Reynolds (turbulenta) deveria estar relacionada com o gradiente local de velocidade do escoamento médio por meio de uma viscosidade associada às características do fluido, do escoamento e da geometria envolvida no problema sob consideração ([FREIRE et al., 2002]). Assim, o modelo para a tensão de Reynolds, proposto por Boussinesq, é

$$
\begin{gathered}
\tau_{z z}^{t}=-{\overline{u^{\prime}}}^{2}=2 \mu_{t} \frac{\partial \bar{u}}{\partial z} \\
\tau_{z r}^{t}=\tau_{r z}^{t}=-\bar{v}^{\prime} \bar{u}^{\prime}=\mu_{t}\left(\frac{\partial \bar{u}}{\partial r}+\frac{\partial \bar{v}}{\partial z}\right),
\end{gathered}
$$


de tal modo que a tensão total passa a ser dada pela soma das tensões devido à difusão molecular e turbulenta do momento:

$$
\begin{gathered}
\tau=\bar{\tau}^{l}+\bar{\tau}^{t} \\
\tau_{z z}=2 \bar{\eta} \frac{\partial \bar{u}}{\partial z}+2 \mu_{t} \frac{\partial \bar{u}}{\partial z}, \\
\tau_{r z}=\left(\bar{\eta}+\mu_{t}\right)\left(\frac{\partial \bar{u}}{\partial r}+\frac{\partial \bar{v}}{\partial z}\right),
\end{gathered}
$$

em que $\mu_{t}$ é a viscosidade dinâmica turbulenta, que é uma propriedade do escoamento, e não do fluido, como a viscosidade molecular.

Das equações (3.2.34) e (3.2.35), a equação (3.2.33) pode ser reescrita como:

$$
\begin{gathered}
\frac{\partial \bar{u}}{\partial t}+\frac{\partial(\bar{u})^{2}}{\partial z}+\frac{1}{r} \frac{\partial(r \bar{v} \bar{v})}{\partial r}=-\frac{1}{\rho} \frac{\partial \bar{p}}{\partial z}+\frac{1}{\rho} \frac{\partial}{\partial z}\left[2\left(\bar{\eta}+\mu_{t}\right) \frac{\partial \bar{u}}{\partial z}\right]+ \\
+\frac{1}{\rho r}\left[\left(\bar{\eta}+\mu_{t}\right)\left(\frac{\partial \bar{u}}{\partial r}+\frac{\partial \bar{v}}{\partial z}\right)\right]+\frac{1}{\rho} \frac{\partial}{\partial r}\left[\left(\bar{\eta}+\mu_{t}\right)\left(\frac{\partial \bar{u}}{\partial r}+\frac{\partial \bar{v}}{\partial z}\right)\right] .
\end{gathered}
$$

Conservação da quantidade de movimento na direção $r$

De forma semelhante ao que foi feito para a obtenção da equação (3.2.39), obteve-se a evolução da velocidade média da componente $r$ :

$$
\begin{gathered}
\frac{\partial \bar{v}}{\partial t}+\frac{\partial(\bar{u} \bar{v})}{\partial z}+\frac{1}{r} \frac{\partial(r \bar{v} \bar{v})}{\partial r}=-\frac{1}{\rho} \frac{\partial \bar{p}}{\partial r}+\frac{1}{\rho} \frac{\partial}{\partial r}\left(2\left(\bar{\eta}+\mu_{t}\right) \frac{\partial \bar{v}}{\partial r}\right)+ \\
+\frac{2\left(\bar{\eta}+\mu_{t}\right)}{\rho r} \frac{\partial \bar{v}}{\partial r}-\frac{2\left(\bar{\eta}+\mu_{t}\right)}{\rho} \frac{\bar{v}}{r^{2}}+\frac{1}{\rho} \frac{\partial}{\partial z}\left[\left(\bar{\eta}+\mu_{t}\right)\left(\frac{\partial \bar{u}}{\partial r}+\frac{\partial \bar{v}}{\partial z}\right)\right] .
\end{gathered}
$$


No contexto da hipótese de Boussinesq, o problema de fechamento da dinâmica média é encontrar uma equação para $\mu_{t}$ em função da posição e do tempo. Por razões dimensionais, a viscosidade turbulenta pode ser calculada utilizando-se a seguinte aproximação:

$$
\mu_{t}=\rho l_{t} u_{t}
$$

em que $u_{t}$ é a escala de velocidade, $\rho$ é a massa específica e $l_{t}$ é o comprimento de turbulência.

Nos modelos de turbulência em que se assume a existência de uma viscosidade adicional, $\mu_{t}$ é descrito algebricamente em função de outras grandezas derivadas de equações diferenciais. Dependendo do número e do tipo das equações empregadas, os modelos são classificados como algébricos, de uma, duas, ou mais equações ([FERREIRA, 2001]). Neste trabalho, utilizou-se o modelo de comprimento de mistura de Prandtl $(1925)^{2}$ (CEBECI and BRADSHAW, 1984]), o qual é um modelo algébrico ou de zero equações; nele, uma expressão algébrica, em função de quantidades médias, é acoplada às equações de conservação da quantidade de movimento para representar a viscosidade turbulenta,

$$
\mu_{t}=\rho l^{2}\left(\frac{\partial \bar{u}}{\partial r}+\frac{\partial \bar{v}}{\partial z}\right)
$$

em que $l$ é o comprimento de mistura, cuja definição é dada por ([CEBECI and BRADSHAW, 1984]):

$$
\frac{l}{R}=0,14-0,08\left(1-\frac{y}{R}\right)^{2}-0,06\left(1-\frac{y}{R}\right)^{4}
$$

\footnotetext{
${ }^{2}$ L. Prandtl em 1925, introduziu o modelo comprimento de mistura e uma maneira direta de se calcular a viscosidade turbulenta. Ele se tornou uma das referências em modelagem de escoamentos turbulentos.
} 
em que $y$ denota a distância normal à parede porosa.

Conservação das espécies químicas

Para a concentração de um composto em um escoamento, substituem-se na equação (3.2.12) as variáveis em termos de seus valores médios e flutuantes e aplica-se o operador média:

$$
\begin{gathered}
\overline{\frac{\partial\left(\bar{c}+c^{\prime}\right)}{\partial t}+\frac{\partial\left(\left(\bar{u}+u^{\prime}\right)\left(\bar{c}+c^{\prime}\right)\right)}{\partial z}+\frac{1}{r} \frac{\partial\left(r\left(\bar{v}+v^{\prime}\right)\left(\bar{c}+c^{\prime}\right)\right)}{\partial r}}= \\
\frac{D\left(\frac{\partial^{2}\left(\bar{c}+c^{\prime}\right)}{\partial r^{2}}+\frac{\partial^{2}\left(\bar{c}+c^{\prime}\right)}{\partial z^{2}}+\frac{1}{r} \frac{\partial\left(\bar{c}+c^{\prime}\right)}{\partial r}\right)}{}
\end{gathered}
$$

Das equações (3.2.15)-(3.2.22), tem-se:

$$
\begin{gathered}
\frac{\partial \bar{c}}{\partial t}+\frac{\partial(\bar{u} \bar{c})}{\partial z}+\frac{\partial\left(\overline{u^{\prime} \bar{c}^{\prime}}\right)}{\partial z}+\frac{1}{r} \frac{\partial(r \bar{v} \bar{c})}{\partial r}+\frac{1}{r} \frac{\partial\left(r \bar{v}^{\prime} \overline{c^{\prime}}\right)}{\partial r}= \\
D\left(\frac{\partial^{2} \bar{c}}{\partial r^{2}}+\frac{\partial^{2} \bar{c}}{\partial z^{2}}+\frac{1}{r} \frac{\partial \bar{c}}{\partial r}\right) .
\end{gathered}
$$

Rearranjando-se os termos, obtém-se:

$$
\frac{\partial \bar{c}}{\partial t}+\frac{\partial(\bar{u} \bar{c})}{\partial z}+\frac{1}{r} \frac{\partial(r \bar{v} \bar{c})}{\partial r}=\frac{\partial}{\partial z}\left(D \frac{\partial \bar{c}}{\partial z}-\bar{u}^{\prime} \bar{c}^{\prime}\right)+\frac{\partial}{\partial r}\left(D \frac{\partial \bar{c}}{\partial r}-\bar{v}^{\prime} \bar{c}^{\prime}\right)+\frac{1}{r}\left(D \frac{\partial \bar{c}}{\partial r}-\bar{v}^{\prime} \bar{c}^{\prime}\right)
$$

Nota-se que as parcelas entre parêntesis na equação (3.2.46) contêm o fluxo molecular de massa, de acordo com a definição da lei de Fick. O segundo termo em cada parêntesis relacionado com as flutuações turbulentas representa, portanto, o fluxo tubulento de massa para cada direção coordenada:

$$
\dot{m}_{z}^{t}=\bar{u}^{\prime} \bar{c}^{\prime}=-D_{t} \frac{\partial \bar{c}}{\partial z}
$$




$$
\dot{m}_{r}^{t}=\bar{v}^{\prime} \bar{c}^{\prime}=-D_{t} \frac{\partial \bar{c}}{\partial r}
$$

Assim, como no caso da equação de conservação da quantidade de movimento, o fluxo total de massa em um escoamento turbulento pode ser expresso por:

$$
\dot{m}=\dot{m}^{l}+\dot{m}^{t}
$$

com

$$
\begin{aligned}
& \dot{m}_{z}=-\left(D+D_{t}\right) \frac{\partial \bar{c}}{\partial z} \\
& \dot{m}_{r}=-\left(D+D_{t}\right) \frac{\partial \bar{c}}{\partial r}
\end{aligned}
$$

em que $D_{t}$ é denominado coeficiente de difusão turbulenta (ou coeficiente de difusão molecular em regime turbulento).

Assim, com (3.2.50) e (3.2.51), pode-se reescrever a equação (3.2.46) como:

$$
\frac{\partial \bar{c}}{\partial t}+\frac{\partial(\bar{u} \bar{c})}{\partial z}+\frac{1}{r} \frac{\partial(r \bar{v} \bar{c})}{\partial r}=\frac{\partial}{\partial z}\left[\left(D+D_{t}\right) \frac{\partial \bar{c}}{\partial z}\right]+\frac{\partial}{\partial r}\left[\left(D+D_{t}\right) \frac{\partial \bar{c}}{\partial r}\right]+\frac{1}{r}\left(D+D_{t}\right) \frac{\partial \bar{c}}{\partial r}
$$

O coeficiente de difusão turbulenta é determinado por uma expressão semelhante à expressão da viscosidade turbulenta ([BENNETT and MYERS, 1978|)(equação (3.2.42) $)$ :

$$
D_{t}=l^{2}\left(\frac{\partial \bar{u}}{\partial r}+\frac{\partial \bar{v}}{\partial z}\right)
$$




\subsubsection{Adimensionalização}

Com o objetivo de tornar os modelos independentes de qualquer sistema de unidades, considera-se a seguinte mudança de variáveis:

$$
\begin{aligned}
& z^{*}=\frac{z}{R}, \quad r^{*}=\frac{r}{R}, \quad l^{*}=\frac{l}{R}, \quad y^{*}=\frac{y}{R} \quad u^{*}=\frac{\bar{u}}{u_{0}}, \quad v^{*}=\frac{\bar{v}}{u_{0}}, \quad p^{*}=\frac{\bar{p}}{\rho u_{0}^{2}}, \\
& t^{*}=\frac{t u_{0}}{R}, \quad c^{*}=\frac{\bar{c}}{c_{0}}, \quad \eta^{*}=\frac{\bar{\eta}}{\eta_{0}}, \quad \mu_{t}^{*}=\frac{\mu_{t}}{\mu_{0}}, \quad D_{t}^{*}=\frac{D_{t}}{D},
\end{aligned}
$$

por conveniência, assume-se $\eta_{0}=m\left(\frac{u_{0}}{R}\right)^{n-1}$ e $\mu_{0}=\eta_{0}$.

Por simplicidade, serão adimensionalizadas as equações que definem o escoamento turbulento. Assim, substituindo-se as transformações (3.2.54) na equação (3.2.39) e desprezandose o símbolo "*"para simplificar a notação, tem-se:

$$
\begin{aligned}
& \frac{u_{0}^{2}}{R} \frac{\partial u}{\partial t}+\frac{u_{0}^{2}}{R} \frac{\partial u^{2}}{\partial z}+\frac{u_{0}^{2}}{R r} \frac{\partial(r u v)}{\partial r}=-\frac{u_{0}^{2}}{R} \frac{\partial p}{\partial z}+\frac{u_{0}}{\rho R^{2}} \frac{\partial}{\partial z}\left[2\left(\frac{u_{0}}{R}\right)^{n-1} m\left(\eta+\mu_{t}\right) \frac{\partial u}{\partial z}\right]+ \\
& \quad+\left(\frac{u_{0}}{R}\right)^{n-1} \frac{m\left(\eta+\mu_{t}\right) u_{0}}{\rho r R^{2}}\left(\frac{\partial u}{\partial r}+\frac{\partial v}{\partial z}\right)+\frac{u_{0}}{\rho R^{2}} \frac{\partial}{\partial r}\left[\left(\frac{u_{0}}{R}\right)^{n-1} m\left(\eta+\mu_{t}\right)\left(\frac{\partial u}{\partial r}+\frac{\partial v}{\partial z}\right)\right] .
\end{aligned}
$$

Fazendo-se a multiplicação de ambos os lados da equação (3.2.55) por $\frac{R}{u_{0}{ }^{2}}$, obtémse a forma adimensionalizada da equação de conservação da quantidade de movimento na direção $z$ para um escoamento turbulento:

$$
\begin{gathered}
\frac{\partial u}{\partial t}+\frac{\partial u^{2}}{\partial z}+\frac{1}{r} \frac{\partial(r u v)}{\partial r}=-\frac{\partial p}{\partial z}+\frac{8}{R e}\left(\frac{n}{3 n+1}\right)^{n}\left\{\frac{\partial}{\partial z}\left[2\left(\eta+\mu_{t}\right) \frac{\partial u}{\partial z}\right]+\right. \\
\left.\frac{\left(\eta+\mu_{t}\right)}{r}\left(\frac{\partial u}{\partial r}+\frac{\partial v}{\partial z}\right)+\frac{\partial}{\partial r}\left[\left(\eta+\mu_{t}\right)\left(\frac{\partial u}{\partial r}+\frac{\partial v}{\partial z}\right)\right]\right\} .
\end{gathered}
$$


As adimensionalizações das outras equações são obtidas de forma análoga.

$$
\begin{gathered}
\frac{\partial v}{\partial t}+\frac{\partial(u v)}{\partial z}+\frac{1}{r} \frac{\partial\left(r v^{2}\right)}{\partial r}=-\frac{\partial p}{\partial r}+\frac{8}{R e}\left(\frac{n}{3 n+1}\right)^{n}\left\{\frac{\partial}{\partial r}\left[2\left(\eta+\mu_{t}\right) \frac{\partial v}{\partial r}\right]+\right. \\
\left.\frac{2}{r}\left(\eta+\mu_{t}\right) \frac{\partial v}{\partial r}-2\left(\eta+\mu_{t}\right) \frac{v}{r^{2}}+\frac{\partial}{\partial z}\left[\left(\eta+\mu_{t}\right)\left(\frac{\partial u}{\partial r}+\frac{\partial v}{\partial z}\right)\right]\right\} \\
\frac{\partial c}{\partial t}+\frac{\partial(u c)}{\partial z}+\frac{1}{r} \frac{\partial(r v c)}{\partial r}=\frac{2}{P e}\left\{\frac{\partial}{\partial z}\left[\left(1+D_{t}\right) \frac{\partial c}{\partial z}\right]+\frac{\partial}{\partial r}\left[\left(1+D_{t}\right) \frac{\partial c}{\partial r}\right]+\frac{1}{r}\left(1+D_{t}\right) \frac{\partial c}{\partial r}\right\} \\
\frac{\partial u}{\partial z}+\frac{1}{r} \frac{\partial r v}{\partial r}=0
\end{gathered}
$$

em que,

$$
\begin{gathered}
\eta=\left[2\left(\frac{\partial u}{\partial z}\right)^{2}+2\left(\frac{\partial v}{\partial r}\right)^{2}+2\left(\frac{v}{r}\right)^{2}+\left(\frac{\partial u}{\partial r}+\frac{\partial v}{\partial z}\right)^{2}\right]^{\frac{n-1}{2}} \\
\mu_{t}=\frac{1}{8}\left(\frac{3 n+1}{n}\right)^{n} \operatorname{Re}^{2}\left(\frac{\partial u}{\partial r}+\frac{\partial v}{\partial z}\right) \\
D_{t}=\frac{P e}{2} l^{2}\left(\frac{\partial u}{\partial r}+\frac{\partial v}{\partial z}\right) \\
l=0,14-0,08(1-y)^{2}-0,06(1-y)^{4}
\end{gathered}
$$

em que $y=1-\frac{r}{R}, R e=\frac{u_{0}^{2-n}(2 R)^{n} \rho}{m 8^{n-1}}\left(\frac{4 n}{3 n+1}\right)^{n}$ é o número de Reynolds generalizado para fluidos não-newtonianos ( CARREAU et al., 1997]) e $P e=\frac{2 R u_{0}}{D}$ é o número de Peclet .

\subsubsection{Condições iniciais e de fronteira}

Em mecânica dos fluidos, os problemas estão sempre relacionados com um domínio, já que o comportamento físico de cada caso depende das condições iniciais e de fronteira 
apropriadas.

\section{Condições iniciais}

As condições iniciais podem ser especificadas por:

$$
u(z, r, t)=u_{0}, \quad v(z, r, t)=v_{0}, \quad p(z, r, t)=p_{0}, \quad c(z, r, t)=c_{0}, \quad t=0
$$

em que $u_{0}, c_{0}$ e $p_{0}$ são constantes.

Condições de fronteira

- entrada: $u(z, r, t)=u_{0}, v(z, r, t)=0, p(z, r, t)=p_{0}$ e $c(z, r, t)=c_{0}$, em que $u_{0}, c_{0}$ e $p_{0}$ são constantes;

- saída: $\frac{\partial u}{\partial z}=0, \frac{\partial v}{\partial z}=0$ e $\frac{\partial c}{\partial z}=0$;

- eixo de simetria: $v=0, \frac{\partial u}{\partial r}=0$ e $\left.\frac{\partial c}{\partial r}\right|_{r=0}=0$;

- parede porosa: $u=0, v=v_{w}$ e $\frac{P e v_{w} c}{2}=\left.\frac{\partial c}{\partial r}\right|_{r=R}$;

em que $v_{w}$ é a velocidade de permeação local, definida pelo modelo de resistência em série ([CHERYAN, 1998, YYH et al., 2003, [DAMAK et al., 2004a], [DAMAK et al., 2004b]), conceituado no capítulo 2, que na forma dimensional é dado por:

$$
v_{w}=\frac{\Delta p}{R_{m}+R_{f}+\phi \Delta p} .
$$

No presente trabalho, os termos $R_{f}$ e $\phi$ são definidos como constantes, pois o objetivo é estudar a transferência de massa e, conseqüentemente, os perfis de concentração, 
quando a camada gel está formada, como mostra a Fig. 3.2. Em alguns resultados, serão usadas as velocidades média de permeação, que são definidas pela expressão:

$$
\bar{v}_{w}=\frac{1}{L} \int_{0}^{L} v_{w}(z, R) d z
$$

em que a integral é resolvida numericamente pela regra do trapézio.

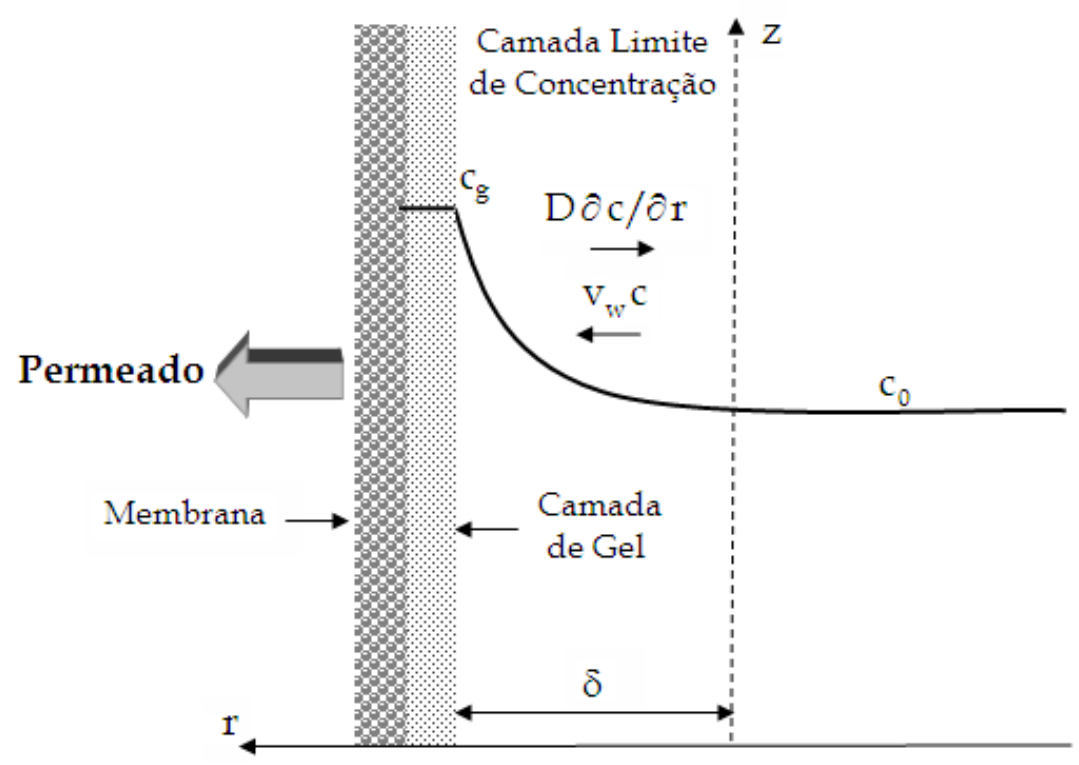

Figura 3.2: Camada de polarização.

Para o comprimento de mistura em regiões próximas à parede, utiliza-se uma equação apropriada para modelar esta região do escoamento: a função de amortecimento de Van Driest ([CEBECI and BRADSHAW, 1984]),

$$
l=\bar{\kappa} y\left[1-\exp \left(-\frac{y}{A}\right)\right],
$$

em que $\bar{\kappa}=0,435$ ([CEBECI and BRADSHAW, 1984]) é a constante de Von Karman, $A$ 
e $A^{+}$são definidos como ([CEBECI and BRADSHAW, 1984]):

$$
\begin{gathered}
A=A^{+} \nu\left(\frac{\tau_{w}}{\rho}\right)^{-1 / 2}, \\
A^{+}=26 \exp \left(-5,9 v_{w}^{+}\right), \\
v_{w}^{+}=\frac{v_{w}}{u_{\tau}}, \\
u_{\tau}=\sqrt{\frac{\tau_{w}}{\rho}},
\end{gathered}
$$

sendo que $\tau_{w}$ é tensão de cisalhamento na parede ([FOX and McDONALD, 1995]):

$$
\tau_{w}=0,03325 \rho u_{0}^{2}\left(\frac{\nu}{d u_{0}}\right)^{0,25}
$$

Com a formulação matemática e as condições de fronteiras definidas, a próxima etapa é a discretização das equações, apresentada no próximo capítulo. 


\section{Capitulo 4}

\section{Discretização das Equações e Método}

\section{Computacional}

Para tratar o modelo computacional, é necessário expressar de forma adequada as equações e o domínio em que elas são válidas. Como não se pode obter soluções numéricas sobre um região contínua, devido aos infinitos pontos da mesma, inicialmente o domínio é discretizado, ou seja, dividido em pontos, nos quais as soluções são obtidas. A esse conjunto de pontos discretos dá-se o nome de malha. A distribuição adequada dos pontos no domínio é fundamental para se obter uma solução numérica representativa do escoamento. Os termos que aparecem nas equações são escritos em função dos valores das incógnitas em pontos discretos adjacentes. O resultado é um conjunto de equações algébricas, geralmente lineares. Essas equações algébricas, juntamente com as condições de fronteira e iniciais, são resolvidas por métodos numéricos ([FORTUNA, 2000]). Neste trabalho, utilizou-se o método Sola ([HIRT et al., 1975, FORTUNA, 2000]), o qual é conceitualmente semelhante ao método MAC ([HARLOW and WELCH, 1965]). A diferença entre os dois métodos 
está basicamente na forma de resolução da equação de Poisson, sendo essa diferença que facilita, no Sola, o tratamento de fronteira de saída de fluido, por isso o Sola é mais indicado para problemas com fronteira de saída.

No presente capítulo, serão abordadas as discretizações das equações apresentadas no capítulo 3, bem como o método de resolução numérica de tais equações.

\subsection{Discretização}

As equações foram discretizadas por diferenças finitas em malha deslocada. Essa malha apresentada em Harlow e Wlech (1965) se tornou um padrão no cálculo de escoamentos incompressíveis. Ela é composta por elementos (células) retangulares de largura $\Delta z$ e altura $\Delta r$, as velocidade $u$ e $v$ estão definidas no centro de cada lado vertical e horizontal de cada elemento, respectivamente; já as componentes da pressão, $p$, concentração, $c$, viscosidade aparente, $\eta$, viscosidade turbulenta, $\mu_{t}$, e difusividade turbulenta, $D_{t}$, estão definidas no centro, como mostra a Fig. 4.1 ([FORTUNA, 2000]):

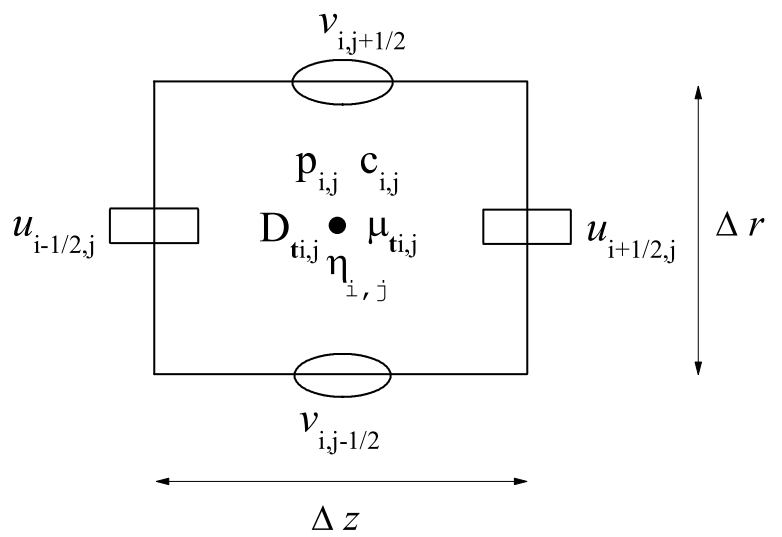

Figura 4.1: Célula computacional da malha deslocada. 


\subsubsection{Discretização das equações que modelam o escoamento}

Como a discretização foi em malha deslocada, a equação de conservação da quantidade de movimento na direção $z$ foi discretizada sobre a posição $(i+1 / 2, j)$; na direção $r$, ela foi discretizada sobre a posição $(i, j+1 / 2)$; as equações de conservação de massa, de conservação das espécies químicas e da viscosidade aparente turbulenta e coeficiente de difusão turbulenta foram discretizadas na posição $(i, j)$.

Nas aproximações para as derivadas temporais, utilizaram-se diferenças avançadas (Euler explícito) de primeira ordem, os gradientes de pressão, bem como a equação de conservação de massa e os outros termos das equações de conservação da quantidade de movimento e das espécies químicas, com exceção dos termos convectivos, uma vez que estes terão uma seção especial para sua discretização.

Em tais aproximações, foram utilizadas diferenças centrais de segunda ordem. Para exemplificar, sejam a derivada temporal, o gradiente de pressão e um termo difusivo, estes últimos pertencentes à equação (3.2.56), ao aproximá-los no ponto $(i+1 / 2, j)$, têm-se, respectivamente:

$$
\left.\frac{\partial u}{\partial t}\right|_{i+1 / 2, j} \approx \frac{u_{i+1 / 2, j}^{N+1}-u_{i+1 / 2, j}^{N}}{\Delta t}
$$

$$
\left.\frac{\partial p}{\partial z}\right|_{i+1 / 2, j} \approx \frac{p_{i+1, j}-p_{i, j}}{\Delta z}
$$

$$
\left.\frac{\partial}{\partial z}\left[2\left(\eta+\mu_{t}\right) \frac{\partial u}{\partial z}\right]\right|_{i+1 / 2, j} \approx\left[\left.2\left(\eta_{i+1, j}+\mu_{t i+1, j}\right) \frac{\partial u}{\partial z}\right|_{i+1, j}-\left.2\left(\eta_{i, j}+\mu_{t i, j}\right) \frac{\partial u}{\partial z}\right|_{i, j}\right] \frac{1}{\Delta z}=
$$




$$
=2\left(\eta_{i+1, j}+\mu_{t i+1, j}\right)\left(\frac{u_{i+3 / 2, j}-u_{i+1 / 2, j}}{(\Delta z)^{2}}\right)-2\left(\eta_{i, j}+\mu_{t i, j}\right)\left(\frac{u_{i+1 / 2, j}-u_{i-1 / 2, j}}{(\Delta z)^{2}}\right) .
$$

Os outros termos difusivos foram aproximados de forma análoga à descrita na equação (4.1.3). Para se evitar repetições, a seguir, serão mostradas as discretizações das equações para o escoamento em regime turbulento, pois o laminar é análogo.

Conservação de massa

$$
\frac{u_{i+1 / 2, j}-u_{i-1 / 2, j}}{\Delta z}+\frac{r_{i, j+1 / 2} v_{i, j+1 / 2}-r_{i, j-1 / 2} v_{i, j-1 / 2}}{r_{i, j} \Delta r}=0,
$$

em que $r_{i, j}=(j-1 / 2) \Delta r$.

Conservação da quantidade de movimento na direção z

$$
\begin{gathered}
u_{i+1 / 2, j}^{N+1}=u_{i+1 / 2, j}+\Delta t\left\{\left(-C O N V_{i+1 / 2, j}^{u}\right)-\frac{p_{i+1, j}-p_{i, j}}{\Delta z}+\frac{16}{R e}\left(\frac{n}{3 n+1}\right)^{n}[\right. \\
\left.\left(\eta_{i+1, j}+\mu_{t i+1, j}\right)\left(\frac{u_{i+3 / 2, j}-u_{i+1 / 2, j}}{(\Delta z)^{2}}\right)-\left(\eta_{i, j}+\mu_{t i, j}\right)\left(\frac{u_{i+1 / 2, j}-u_{i-1 / 2, j}}{(\Delta z)^{2}}\right)\right]+ \\
\frac{8}{R e}\left(\frac{n}{3 n+1}\right)^{n}\left[\left(\frac{\eta_{i+1 / 2, j}+\mu_{t i+1 / 2, j}}{r_{i+1 / 2, j}}\right)\left(\frac{u_{i+1 / 2, j+1 / 2}-u_{i+1 / 2, j-1 / 2}}{\Delta r}+\frac{v_{i+1, j}-v_{i, j}}{\Delta z}\right)+\right. \\
\left(\eta_{i+1 / 2, j+1 / 2}+\mu_{t i+1 / 2, j+1 / 2}\right)\left(\frac{u_{i+1 / 2, j+1}-u_{i+1 / 2, j}}{(\Delta r)^{2}}+\frac{v_{i+1, j+1 / 2}-v_{i, j+1 / 2}}{\Delta r \Delta z}\right)- \\
\left.\left.\left(\eta_{i+1 / 2, j-1 / 2}+\mu_{t i+1 / 2, j-1 / 2}\right)\left(\frac{u_{i+1 / 2, j}-u_{i+1 / 2, j-1}}{(\Delta r)^{2}}+\frac{v_{i+1, j-1 / 2}-v_{i, j-1 / 2}}{\Delta r \Delta z}\right)\right]\right\}
\end{gathered}
$$


Conservação da quantidade de movimento na direção $r$

$$
\begin{gathered}
v_{i, j+1 / 2}^{N+1}=v_{i, j+1 / 2}^{n}+\Delta t\left\{-C O N V_{i, j+1 / 2}^{v}-\frac{p_{i, j+1}-p_{i, j}}{\Delta r}+\frac{16}{R e}\left(\frac{n}{3 n+1}\right)^{n}[\right. \\
\left(\eta_{i, j+1}+\mu_{t i, j+1}\right)\left(\frac{v_{i, j+3 / 2}-v_{i, j+1 / 2}}{(\Delta r)^{2}}\right)-\left(\eta_{i, j}+\mu_{t i, j}\right)\left(\frac{v_{i, j+1 / 2}-v_{i, j-1 / 2}}{(\Delta r)^{2}}\right)+ \\
\left.\left(\eta_{i, j+1 / 2}+\mu_{t i, j+1 / 2}\right)\left(\frac{v_{i, j+1}-v_{i, j}}{\Delta r}\right)-\left(\eta_{i, j+1 / 2}+\mu_{t i, j+1 / 2}\right) \frac{v_{i, j+1 / 2}}{r_{i, j+1 / 2}^{2}}\right]+ \\
\frac{8}{R e}\left(\frac{n}{3 n+1}\right)^{n}\left[\left(\eta_{i+1 / 2, j+1 / 2}+\mu_{t i+1 / 2, j+1 / 2}\right)\left(\frac{u_{i+1 / 2, j+1}-u_{i+1 / 2, j}}{\Delta r \Delta z}+\frac{v_{i+1, j+1 / 2}-v_{i, j+1 / 2}}{(\Delta z)^{2}}\right)-\right. \\
\left.\left(\eta_{i-1 / 2, j+1 / 2}+\mu_{t i-1 / 2, j+1 / 2}\right)\left(\frac{u_{i-1 / 2, j+1}-u_{i-1 / 2, j}}{\Delta r \Delta z}+\frac{v_{i, j+1 / 2}-v_{i-1, j+1 / 2}}{(\Delta z)^{2}}\right)\right]
\end{gathered}
$$

em que $\Delta t$ é o passo no tempo e $C O N V_{i+1 / 2, j}^{u}$ e $C O N V_{i, j+1 / 2}^{v}$ são os termos convectivos na direção $z$ e $r$, respectivamente:

$$
\begin{gathered}
C O N V_{i+1 / 2, j}^{u}=\left.\left(\frac{\partial u^{2}}{\partial z}+\frac{1}{r} \frac{\partial(r u v)}{\partial r}\right)\right|_{i+1 / 2, j}, \\
C O N V_{i, j+1 / 2}^{v}=\left.\left(\frac{\partial(u v)}{\partial z}+\frac{1}{r} \frac{\partial\left(r v^{2}\right)}{\partial r}\right)\right|_{i, j+1 / 2} .
\end{gathered}
$$

Observa-se que a viscosidade aparente e a viscosidade turbulenta aparecem avaliadas em pontos da células em que essas viscosidades não são conhecidas. Nesses casos, esses termos foram aproximados utilizando-se uma média dos quatro valores vizinhos mais próximos, ou fazendo-se uma média aritmética, dependendo do caso. Assim, tem-se:

$$
\eta_{i+1 / 2, j}=\frac{\eta_{i, j}+\eta_{i+1, j}}{2}
$$




$$
\begin{gathered}
\eta_{i, j+1 / 2}=\frac{\eta_{i, j}+\eta_{i, j+1}}{2}, \\
\eta_{i+1 / 2, j+1 / 2}=\frac{\eta_{i, j}+\eta_{i+1, j}+\eta_{i, j+1}+\eta_{i+1, j+1}}{4}, \\
\eta_{i+1 / 2, j-1 / 2}=\frac{\eta_{i, j}+\eta_{i+1, j}+\eta_{i, j-1}+\eta_{i+1, j-1}}{4}, \\
\eta_{i-1 / 2, j+1 / 2}=\frac{\eta_{i-1, j+1}+\eta_{i, j+1}+\eta_{i-1, j}+\eta_{i, j}}{4}, \\
\mu_{t i+1 / 2, j}=\frac{\mu_{t i, j}+\mu_{t i+1, j}}{2}, \\
\mu_{t i+1 / 2, j+1 / 2}=\frac{\mu_{t i, j}+\mu_{t i, j+1}}{2}, \\
\mu_{t i+1 / 2, j-1 / 2}=\frac{\mu_{t i, j}+\mu_{t i+1, j}+\mu_{t i, j-1}+\mu_{t i+1, j-1}}{4}, \\
\mu_{t i-1 / 2, j+1 / 2}+\mu_{t i, j+1}+\mu_{t i+1, j+1} \\
=\frac{\mu_{t i-1, j+1}+\mu_{t i, j+1}+\mu_{t i-1, j}+\mu_{t i, j}}{4}
\end{gathered}
$$

O mesmo se aplica para as velocidades:

$$
\begin{gathered}
u_{i+1 / 2, j+1 / 2}=\frac{u_{i+1 / 2, j}+u_{i+1 / 2, j+1}}{2}, \\
u_{i+1 / 2, j-1 / 2}=\frac{u_{i+1 / 2, j}+u_{i+1 / 2, j-1}}{2}, \\
v_{i+1, j}=\frac{v_{i+1, j+1 / 2}+v_{i+1, j-1 / 2}}{2}, \\
v_{i, j}=\frac{v_{i, j+1 / 2}+v_{i, j-1 / 2}}{2}, \\
v_{i, j+1}=\frac{v_{i, j+3 / 2}+v_{i, j+1 / 2}}{2} .
\end{gathered}
$$


Conservação das espécies químicas

$c_{i, j}^{N+1}=c_{i, j}+\delta t\left\{-C O N V_{i, j}^{c}+\frac{2}{P e}\left[\left(1+D_{t i+1 / 2, j}\right)\left(\frac{c_{i+1, j}-c_{i, j}}{(\Delta z)^{2}}\right)-\left(1+D_{t i-1 / 2, j}\right)\left(\frac{c_{i, j}-c_{i-1, j}}{(\Delta z)^{2}}\right)+\right.\right.$

$\left.\left.\left(1+D_{t i, j+1 / 2}\right)\left(\frac{c_{i, j+1}-c_{i, j}}{(\Delta r)^{2}}\right)-\left(1+D_{t i, j-1 / 2}\right)\left(\frac{c_{i, j}-c_{i, j-1}}{(\Delta r)^{2}}\right)+\frac{\left(1+D_{t i, j}\right)}{r_{i, j}}\left(\frac{c_{i, j+1 / 2}-c_{i, j-1 / 2}}{\Delta r}\right)\right]\right\}$

em que,

$$
\begin{aligned}
C O N V_{i, j}^{c}=\left.\frac{\partial(u c)}{\partial z}\right|_{i, j}+\left.\frac{1}{r} \frac{\partial(r v c)}{\partial r}\right|_{i, j} \\
c_{i, j+1 / 2}=\frac{c_{i, j}+c_{i, j+1}}{2}, \\
c_{i, j-1 / 2}=\frac{c_{i, j}+c_{i, j-1}}{2}, \\
D_{t i-1 / 2, j}=\frac{D_{t i, j}+D_{t i-1, j}}{2}, \\
D_{t i+1 / 2, j}=\frac{D_{t i, j}+D_{t i+1, j}}{2}, \\
D_{t i, j-1 / 2}=\frac{D_{t i, j}+D_{t i, j-1}}{2}, \\
D_{t i, j+1 / 2}=\frac{D_{t i, j}+D_{t i, j+1}}{2} .
\end{aligned}
$$

Viscosidade aparente

$$
\begin{gathered}
\eta_{i, j}=\left\{2\left[\left(\frac{u_{i+1 / 2, j}-u_{i-1 / 2, j}}{\Delta z}\right)^{2}+\left(\frac{v_{i, j+1 / 2}-v_{i, j-1 / 2}}{\Delta r}\right)^{2}+\left(\frac{v_{i, j}}{r_{i, j}}\right)^{2}\right]+\right. \\
\left.\left(\frac{u_{i, j+1 / 2}-u_{i, j-1 / 2}}{\Delta r}+\frac{v_{i+1 / 2, j}-v_{i-1 / 2, j}}{\Delta z}\right)^{2}\right\}^{\frac{n-1}{2}} .
\end{gathered}
$$


Viscosidade turbulenta

$$
\mu_{t i, j}=\frac{1}{8}\left(\frac{3 n+1}{n}\right)^{n} \operatorname{Re}_{i, j}^{2}\left(\frac{u_{i, j+1 / 2}-u_{i, j-1 / 2}}{\Delta r}+\frac{v_{i+1 / 2, j}-v_{i-1 / 2, j}}{\Delta z}\right) .
$$

Difusividade turbulenta

$$
D_{t i, j}=\frac{P e}{2} l_{i, j}^{2}\left(\frac{u_{i, j+1 / 2}-u_{i, j-1 / 2}}{\Delta r}+\frac{v_{i+1 / 2, j}-v_{i-1 / 2, j}}{\Delta z}\right)
$$

em que,

$$
\begin{gathered}
l_{i, j}=0,14-0,08\left(1-y_{i, j}\right)^{2}-0,06\left(1-y_{i, j}\right)^{4}, \\
y_{i, j}=1-\frac{r_{i, j}}{R}, \\
u_{i, j+1 / 2}=\frac{u_{i+1 / 2, j}+u_{i-1 / 2, j}+u_{i-1 / 2, j+1}+u_{i-1 / 2, j+1}}{4}, \\
u_{i, j-1 / 2}=\frac{u_{i+1 / 2, j}+u_{i-1 / 2, j}+u_{i+1 / 2, j-1}+u_{i-1 / 2, j-1}}{4}, \\
v_{i+1 / 2, j}=\frac{v_{i, j+1 / 2}+v_{i+1, j+1 / 2}+v_{i, j-1 / 2}+v_{i+1, j-1 / 2}}{4}, \\
v_{i-1 / 2, j}=\frac{v_{i-1, j+1 / 2}+v_{i, j+1 / 2}+v_{i-1, j-1 / 2}+v_{i, j-1 / 2}}{4} .
\end{gathered}
$$

A discretização por diferenças centrais de alguns termos difusivos das equações de conservação da quantidade de movimento e das espécies químicas, quando feita em células adjacentes às fronteiras, produz resultados que exigem a especificação de valores fora do domínio computacional, como, por exemplo, o caso da discretização do termo

$$
\left.\frac{\partial}{\partial r}\left[\left(1+D_{t}\right) \frac{\partial c}{\partial r}\right]\right|_{i, j} \approx\left(1+D_{t i, j+1 / 2}\right)\left(\frac{c_{i, j+1}-c_{i, j}}{(\Delta r)^{2}}\right)-\left(1+D_{t i, j-1 / 2}\right)\left(\frac{c_{i, j}-c_{i, j-1}}{(\Delta r)^{2}}\right) .
$$


Considerando-se $(i, j)$ adjacente à parede porosa, tem-se que $c_{i, j+1}$ está fora do domínio, como pode-se observar na Fig. 4.2. Uma prática atraente e de fácil aplicação para se resolver esse problema é o uso de células fictícias. Nesse caso, tomam-se as células $(i, j+1)$ como sendo aquelas que fazem parte do domínio. Definindo-se a concentração na parede porosa, $c_{w}$, a qual possui valor conhecido, como:

$$
c_{w}=\frac{c_{i, j}+c_{i, j+1}}{2}
$$

Tem-se, então, $c_{i, j+1}=2 c_{w}-c_{i, j}$.

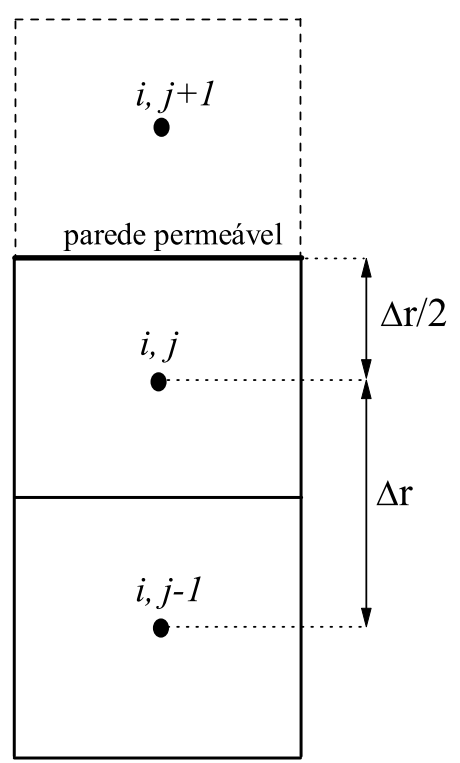

Figura 4.2: Célula adjacente à parede porosa.

Portanto,

$$
\left.\frac{\partial}{\partial r}\left[\left(1+D_{t}\right) \frac{\partial c}{\partial r}\right]\right|_{i, j} \approx\left(1+D_{t i, j+1 / 2}\right)\left(\frac{2 c_{w}-2 c_{i, j}}{(\Delta r)^{2}}\right)-\left(1+D_{t i, j-1 / 2}\right)\left(\frac{c_{i, j}-c_{i, j-1}}{(\Delta r)^{2}}\right)
$$

Para casos semelhantes aos da equação (4.1.13), aplicou-se procedimento análogo. 


\subsubsection{Aproximação dos termos convectivos}

Em escoamentos nos quais a convecção tem papel importante, a discretização adequada dos termos convectivos é de extrema importância para a qualidade da solução numérica, pois esses termos são os responsáveis por muitos fenômenos complexos no escoamento e os principais causadores de dificuldades numéricas nas simulações. A escolha do tipo de discretização pode gerar oscilações não físicas e conseqüentemente acarretar instabilidade numérica. O exemplo mais comum é a difusão numérica ou artificial, introduzida pela aproximação upwind. Por isso, um dos maiores desafios na Dinâmica dos Fluidos Compucional (DFC) é encontrar aproximações para os termos convectivos que não introduzam distorções na solução numérica. Por essa razão, várias técnicas de discretização têm sido desenvolvidas nos últimos anos.

O desenvolvimento de esquemas upwind para aproximar termos convectivos (em geral não-lineares) em equações de convecção-difusão tem sido um importante assunto nas últimas três décadas (ver, por exemplo, DARU and TENAUD, 2001, ALHUMAIZI, 2004, WATERSON and DECONINCK, 2007]). No caso particular dos problemas de escoamentos de fluidos, os esquemas de diferenças finitas upwind de primeira ordem e Lax-Friedrichs podem produzir erros significativos, sendo que um dos mais sérios é a produção de efeitos difusivos com o aumento do efeito de viscosidade. Por outro lado, esquemas de alta ordem (segunda ordem ou de ordem maior), tais como, diferenças centrais, SOU 1 ([FROMM, 1968]), QUICK $^{2]}([$ LEONARD, 1979]) e Lax-Wendroff ([LAX and WENDROFF, 1960]) são técnicas populares para aproximação desses termos. Mas, o uso desses esquemas nas vizinhanças de gradientes elevados, normalmente introduz efeitos dispersivos, os quais levam a oscilações

\footnotetext{
${ }^{1}$ Second Order Upwind.

${ }^{2}$ Quadratic Upstream Interpolation for Convective Kinematics.
} 
numéricas, que conduzem à instabilidade numérica.

O comportamento oscilatório da solução numérica próximo a gradientes elevados pode ser evitado com o uso de esquemas upwind que além de alta ordem, possuem as seguintes propriedades atrativas:

(i) são pelo menos de segunda ordem de precisão em regiões onde a solução é suave;

(ii) evitam oscilações não físicas próximas a descontinuidades;

(iii) introduzem pouca difusão numérica;

(iv) não necessitam de viscosidade artificial;

(v) levam em conta a informação física na formulação do esquema e

(vi) incorporam o esquema upwind de primeira ordem na formulação.

Exemplos representativos dos esquemas que tentam levar em conta esses requesitos são:

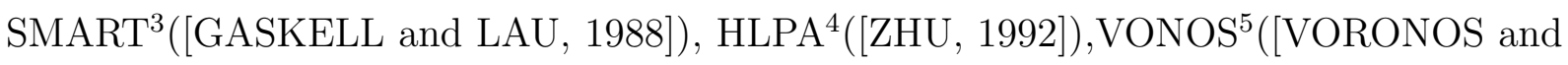

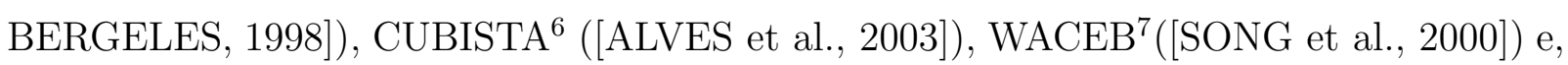
o mais recente, QUICKEST ${ }^{8}$ adaptativo $\left([\text { FERREIRA et al., 2007] })^{9}\right.$.

Considerando-se a importância do esquema convectivo nos resultados numéricos, neste problema, teve-se como um dos objetivos averiguar o desempenho de alguns esquemas convectivos no problema de escoamentos em tubos permeáveis, uma vez que na literatura não se encontraram propostas semelhantes para esse tipo de escoamento. Neste

\footnotetext{
${ }^{3}$ Sharp and Monotonic Algorithm for Realistic Transport.

4 Hybrid-Linear Parabolic Approximation.

${ }^{5}$ Variable-Order Non-Oscillatory Scheme.

${ }^{6}$ Convergent and Universally Bounded Interpolation Scheme for the Treatment of Advection.

${ }^{7}$ Weighted Average Coefficients Ensuring Boundedness.

${ }^{8}$ Quadratic Upstream Interpolation for Convective Kinematics with Estimated Streaming Terms.

${ }^{9}$ Maiores detalhes sobre estes esquemas podem também serem encontrados em Ferreira (2001) e Brandi (2005).
} 
trabalho, dentre os esquemas citados, optou-se por implementar e analisar os esquemas: WACEB, CUBISTA e QUICKEST adaptativo.

Para ilustrar os esquemas, considera-se a Fig. 4.3, em que $v_{f}$ é a velocidade de conveç̧ão na face $f([$ FERREIRA et al., 2007]); $\Delta s$ é o espaçamento dado em um dos eixos coordenados; e os pontos $D$ (à jusante $)^{10}, U$ (à montante $)^{11}$ e $R$ (o mais à montante ${ }^{12}$ são definidos de acordo com $v_{f}$ na face $f$.

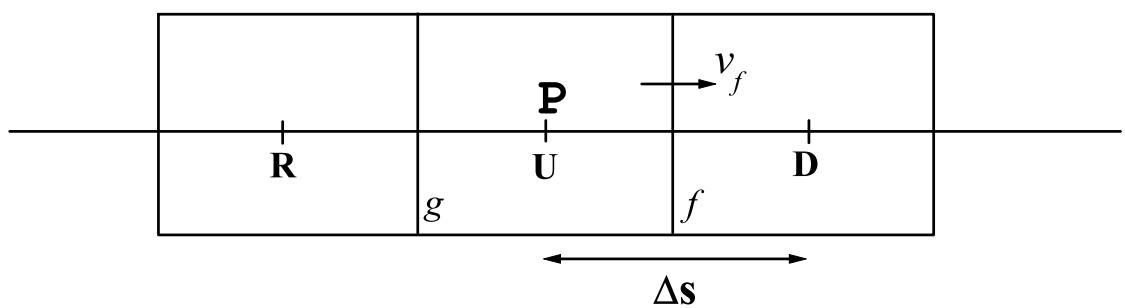

Figura 4.3: Representação esquemática para se obter aproximações dos termos convectivos.

Suponha-se que se deseje aproximar o termo $\frac{\partial(u \phi)}{\partial s}$ em um ponto $\mathbf{P}$, em que $\phi$ representa uma das variáveis $u, v$ ou $c$. Tem-se então:

$$
\left.\frac{\partial(u \phi)}{\partial s}\right|_{P} \approx \frac{\left.(u \phi)\right|_{f}-\left.(u \phi)\right|_{g}}{\Delta s}
$$

em que $u_{f}$ e $u_{g}$ são estimados usando-se a média aritmética dos valores vizinhos $U$ e $D$ para a face $f$ e $U$ e $R$ para a face $g$, quando não se dispõe dos seus valores nessas faces.

Empregando-se os esquema WACEB, CUBISTA e QUICKEST adaptativo para aproximar o termo $\phi_{f}$, têm-se:

\footnotetext{
${ }^{10}$ Downstream.

${ }^{11}$ Upstream.

${ }^{12}$ Remote-upstream.
} 
- WACEB

$$
\phi_{f}= \begin{cases}\phi_{U} & \text { se } \hat{\phi}_{U} \notin[0,1] \\ 2 \phi_{U}-\phi_{R} & \text { se } 0 \leq \hat{\phi}_{U}<3 / 10 \\ \frac{1}{8}\left(3 \phi_{D}+6 \phi_{U}-\phi_{R}\right) & \text { se } 3 / 10 \leq \hat{\phi}_{U} \leq 5 / 6 \\ \phi_{D} & \text { se } 5 / 6<\hat{\phi}_{U} \leq 1,\end{cases}
$$

- CUBISTA

$$
\phi_{f}= \begin{cases}\phi_{U} & \text { se } \hat{\phi}_{U} \notin[0,1], \\ \frac{1}{4}\left(7 \phi_{U}-3 \phi_{R}\right) & \text { se } 0 \leq \hat{\phi}_{U}<3 / 8, \\ \frac{1}{8}\left(3 \phi_{D}+6 \phi_{U}-\phi_{R}\right) & \text { se } 3 / 8 \leq \hat{\phi}_{U} \leq 3 / 4, \\ \frac{1}{4}\left(3 \phi_{D}+\phi_{U}\right) & \text { se } 3 / 4<\hat{\phi}_{U} \leq 1,\end{cases}
$$

- QUICKEST adaptativo

$$
\phi_{f}= \begin{cases}\phi_{U} & \text { se } \hat{\phi}_{U} \notin(0,1), \\ (2-\theta) \phi_{U}-(1-\theta) \phi_{R} & \text { se } 0 \leq \hat{\phi}_{U}<a, \\ \left(\frac{5+3|\theta|-2 \theta^{2}}{6}\right) \phi_{U}+\left(\frac{2-3|\theta|+\theta^{2}}{6}\right) \phi_{D}+\left(\frac{-1}{6}\left(1-\theta^{2}\right)\right) \phi_{R} & \text { se } a \leq \hat{\phi}_{U} \leq b, \\ (1-\theta) \phi_{D}+\theta \phi_{U} & \text { se } b<\hat{\phi}_{U} \leq 1,\end{cases}
$$

em que

$\theta=u_{f} \frac{\Delta t}{\Delta s}, \quad a=\frac{2-3|\theta|+\theta^{2}}{7-6 \theta-3|\theta|+2 \theta^{2}}, \quad b=\frac{-4+6 \theta-3|\theta|+\theta^{2}}{-5+6 \theta-3|\theta|+2 \theta^{2}}, \quad \hat{\phi}_{()}=\frac{\phi_{()}-\phi_{R}}{\phi_{D}-\phi_{R}}$.

O termo $\hat{\phi}_{U}$ é a variável normalizada de Leonard ([LEONARD, 1988, FERREIRA, 2001]). 
De forma análoga obtêm-se as discretizações quando $v_{f}<0$.

Por simplicidade e sem perda de generalidade, considera-se agora na aproximação (4.1.14) a variável $\phi=u$ transportada com velocidade $u$ na direção $z$ no ponto $(i+1 / 2, j)$,

$$
\left.\frac{\partial(u u)}{\partial z}\right|_{i+1 / 2, j} \approx \frac{\left.(\bar{u} u)\right|_{i+1, j}-\left.(\bar{u} u)\right|_{i, j}}{\Delta z}
$$

em que os valores de $\bar{u}_{i+1, j}$ e $\bar{u}_{i, j}$ são aproximados por médias aritméticas:

$$
\bar{u}_{i+1, j}=\frac{u_{i+1 / 2, j}+u_{i+3 / 2, j}}{2}, \quad \bar{u}_{i, j}=\frac{u_{i-1 / 2, j}+u_{i+1 / 2, j}}{2} .
$$

Na equação (4.1.18), os valores da propriedade transportada $u$ nos pontos $(i+1, j)$ e $(i, j)$ não estão disponíveis, assim eles são aproximados, utilizando-se esquemas (4.1.15), (4.1.16) e (4.1.17) e os pontos vizinhos $D, U$ e $R$, os quais são definidos de acordo com a direção do escoamento (sinais de $\bar{u}_{i+1, j}$ e $\bar{u}_{i, j}$ ). Portanto, as aproximações para os termos $u_{i+1, j}$ e $u_{i, j}$ são:

- Para $\bar{u}_{i+1, j}>0$

$$
D=(i+3 / 2, j), U=(i+1 / 2, j), \quad R=(i-1 / 2, j), \hat{u}_{i+1 / 2, j}=\frac{u_{i+1 / 2, j}-u_{i-1 / 2, j}}{u_{i+3 / 2, j}-u_{i-1 / 2, j}}
$$

\section{WACEB}

$$
u_{i+1, j}= \begin{cases}u_{i+1 / 2, j} & \text { se } \hat{u}_{i+1 / 2, j} \notin[0,1], \\ 2 u_{i+1 / 2, j}-u_{i-1 / 2, j} & \text { se } 0 \leq \hat{u}_{i+1 / 2, j}<3 / 10, \\ \frac{1}{8}\left(3 u_{i+3 / 2, j}+6 u_{i+1 / 2, j}-u_{i-1 / 2, j}\right) & \text { se } 3 / 10 \leq \hat{u}_{i+1 / 2, j} \leq 5 / 6, \\ u_{i+3 / 2, j} & \text { se } 5 / 6<\hat{u}_{i+1 / 2, j} \leq 1,\end{cases}
$$




\section{CUBISTA}

$$
u_{i+1, j}= \begin{cases}u_{i+1 / 2, j} & \text { se } \hat{u}_{i+1 / 2, j} \notin[0,1], \\ \frac{1}{4}\left(7 u_{i+1 / 2, j}-3 u_{i-1 / 2, j}\right) & \text { se } 0 \leq \hat{u}_{i+1 / 2, j}<3 / 8, \\ \frac{1}{8}\left(3 u_{i+3 / 2, j}+6 u_{i+1 / 2, j}-u_{i-1 / 2, j}\right) & \text { se } 3 / 8 \leq \hat{u}_{i+1 / 2, j} \leq 3 / 4, \\ \frac{1}{4}\left(3 u_{i+3 / 2, j}+u_{i+1 / 2, j}\right) & \text { se } 3 / 4<\hat{u}_{i+1 / 2, j} \leq 1,\end{cases}
$$

QUICKEST adaptativo

$$
u_{i+1, j}= \begin{cases}u_{i+1 / 2, j} & \text { se } \hat{u}_{i+1 / 2, j} \notin(0,1), \\ (2-\theta) u_{i+1 / 2, j}-(1-\theta) u_{i-1 / 2, j} & \text { se } 0 \leq \hat{u}_{i+1 / 2, j}<a, \\ \alpha_{U} u_{i+1 / 2, j}+\alpha_{D} u_{i+3 / 2, j}+\alpha_{R} u_{i-1 / 2, j} & \text { se } a \leq \hat{u}_{i+1 / 2, j} \leq b, \\ (1-\theta) u_{i+3 / 2, j}+\theta u_{i+1 / 2, j} & \text { se } b<\hat{u}_{i+1 / 2, j} \leq 1,\end{cases}
$$

em que

$$
\alpha_{U}=\frac{5+3|\theta|-2 \theta^{2}}{6}, \quad \alpha_{D}=\frac{2-3|\theta|+\theta^{2}}{6}, \quad \alpha_{R}=\frac{\theta^{2}-1}{6}
$$

- Para $\bar{u}_{i+1, j}<0$

$$
D=(i+1 / 2, j), U=(i+3 / 2, j) \text { e } R=(i+5 / 2, j), \hat{u}_{i+3 / 2, j}=\frac{u_{i+3 / 2, j}-u_{i+5 / 2, j}}{u_{i+1 / 2, j}-u_{i+5 / 2, j}}
$$


WACEB

$$
u_{i+1, j}= \begin{cases}u_{i+3 / 2, j} & \text { se } \hat{u}_{i+1 / 2, j} \notin[0,1], \\ 2 u_{i+3 / 2, j}-u_{i+5 / 2, j} & \text { se } 0 \leq \hat{u}_{i+3 / 2, j}<3 / 10, \\ \frac{1}{8}\left(3 u_{i+1 / 2, j}+6 u_{i+3 / 2, j}-u_{i+5 / 2, j}\right) & \text { se } 3 / 10 \leq \hat{u}_{i+3 / 2, j} \leq 5 / 6, \\ u_{i+1 / 2, j} & \text { se } 5 / 6<\hat{u}_{i+3 / 2, j} \leq 1,\end{cases}
$$

\section{CUBISTA}

$$
u_{i+1, j}= \begin{cases}u_{i+3 / 2, j} & \text { se } \hat{u}_{i+3 / 2, j} \notin[0,1], \\ \frac{1}{4}\left(7 u_{i+3 / 2, j}-3 u_{i+5 / 2, j}\right) & \text { se } 0 \leq \hat{u}_{i+3 / 2, j}<3 / 8, \\ \frac{1}{8}\left(3 u_{i+1 / 2, j}+6 u_{i+3 / 2, j}-u_{i+5 / 2, j}\right) & \text { se } 3 / 8 \leq \hat{u}_{i+1 / 2, j} \leq 3 / 4, \\ \frac{1}{4}\left(3 u_{i+1 / 2, j}+u_{i+3 / 2, j}\right) & \text { se } 3 / 4<\hat{u}_{i+3 / 2, j} \leq 1,\end{cases}
$$

QUICKEST adaptativo

$$
u_{i+1, j}= \begin{cases}u_{i+3 / 2, j} & \text { se } \hat{u}_{i+3 / 2, j} \notin(0,1), \\ (2-\theta) u_{i+3 / 2, j}-(1-\theta) u_{i+5 / 2, j} & \text { se } 0 \leq \hat{u}_{i+3 / 2, j}<a, \\ \alpha_{U} u_{i+3 / 2, j}+\alpha_{D} u_{i+1 / 2, j}+\alpha_{R} u_{i+5 / 2, j} & \text { se } a \leq \hat{u}_{i+3 / 2, j} \leq b, \\ (1-\theta) u_{i+1 / 2, j}+\theta u_{i+3 / 2, j} & \text { se } b<\hat{u}_{i+3 / 2, j} \leq 1,\end{cases}
$$

- Para $\bar{u}_{i, j}>0$

$$
D=(i+1 / 2, j), U=(i-1 / 2, j), \quad R=(i-3 / 2, j), \hat{u}_{i-1 / 2, j}=\frac{u_{i-1 / 2, j}-u_{i-3 / 2, j}}{u_{i+1 / 2, j}-u_{i-3 / 2, j}}
$$


WACEB

$$
u_{i, j}= \begin{cases}u_{i-1 / 2, j} & \text { se } \hat{u}_{i-1 / 2, j} \notin[0,1], \\ 2 u_{i-1 / 2, j}-u_{i-3 / 2, j} & \text { se } 0 \leq \hat{u}_{i-1 / 2, j}<3 / 10, \\ \frac{1}{8}\left(3 u_{i+1 / 2, j}+6 u_{i-1 / 2, j}-u_{i-3 / 2, j}\right) & \text { se } 3 / 10 \leq \hat{u}_{i-1 / 2, j} \leq 5 / 6, \\ u_{i+1 / 2, j} & \text { se } 5 / 6<\hat{u}_{i-1 / 2, j} \leq 1,\end{cases}
$$

\section{CUBISTA}

$$
u_{i, j}= \begin{cases}u_{i-1 / 2, j} & \text { se } \hat{u}_{i-1 / 2, j} \notin[0,1], \\ \frac{1}{4}\left(7 u_{i-1 / 2, j}-3 u_{i-3 / 2, j}\right) & \text { se } 0 \leq \hat{u}_{i-1 / 2, j}<3 / 8, \\ \frac{1}{8}\left(3 u_{i+1 / 2, j}+6 u_{i-1 / 2, j}-u_{i-3 / 2, j}\right) & \text { se } 3 / 8 \leq \hat{u}_{i-1 / 2, j} \leq 3 / 4, \\ \frac{1}{4}\left(3 u_{i+1 / 2, j}+u_{i-1 / 2, j}\right) & \text { se } 3 / 4<\hat{u}_{i-1 / 2, j} \leq 1,\end{cases}
$$

QUICKEST adaptativo

$$
u_{i, j}= \begin{cases}u_{i-1 / 2, j} & \text { se } \hat{u}_{i-1 / 2, j} \notin(0,1), \\ (2-\theta) u_{i-1 / 2, j}-(1-\theta) u_{i-3 / 2, j} & \text { se } 0 \leq \hat{u}_{i-1 / 2, j}<a, \\ \alpha_{U} u_{i-1 / 2, j}+\alpha_{D} u_{i+1 / 2, j}+\alpha_{R} u_{i-3 / 2, j} & \text { se } a \leq \hat{u}_{i-1 / 2, j} \leq b, \\ (1-\theta) u_{i+1 / 2, j}+\theta u_{i-1 / 2, j} & \text { se } b<\hat{u}_{i-1 / 2, j} \leq 1,\end{cases}
$$

- Para $\bar{u}_{i, j}<0$

$$
D=(i-1 / 2, j), U=(i+1 / 2, j), \quad R=(i+3 / 2, j), \hat{u}_{i+1 / 2, j}=\frac{u_{i+1 / 2, j}-u_{i+3 / 2, j}}{u_{i-1 / 2, j}-u_{i+3 / 2, j}},
$$


WACEB

$$
u_{i, j}= \begin{cases}u_{i+1 / 2, j} & \text { se } \hat{u}_{i+1 / 2, j} \notin[0,1], \\ 2 u_{i+1 / 2, j}-u_{i+3 / 2, j} & \text { se } 0 \leq \hat{u}_{i+1 / 2, j}<3 / 10, \\ \frac{1}{8}\left(3 u_{i-1 / 2, j}+6 u_{i+1 / 2, j}-u_{i+3 / 2, j}\right) & \text { se } 3 / 10 \leq \hat{u}_{i+1 / 2, j} \leq 5 / 6, \\ u_{i-1 / 2, j} & \text { se } 5 / 6<\hat{u}_{i+1 / 2, j} \leq 1,\end{cases}
$$

CUBISTA

$$
u_{i, j}= \begin{cases}u_{i+1 / 2, j} & \text { se } \hat{u}_{i+1 / 2, j} \notin[0,1], \\ \frac{1}{4}\left(7 u_{i+1 / 2, j}-3 u_{i+3 / 2, j}\right) & \text { se } 0 \leq \hat{u}_{i+1 / 2, j}<3 / 8, \\ \frac{1}{8}\left(3 u_{i-1 / 2, j}+6 u_{i+1 / 2, j}-u_{i+3 / 2, j}\right) & \text { se } 3 / 8 \leq \hat{u}_{i+1 / 2, j} \leq 3 / 4, \\ \frac{1}{4}\left(3 u_{i-1 / 2, j}+u_{i+1 / 2, j}\right) & \text { se } 3 / 4<\hat{u}_{i+1 / 2, j} \leq 1,\end{cases}
$$

QUICKEST adaptativo

$$
u_{i, j}= \begin{cases}u_{i+1 / 2, j} & \text { se } \hat{u}_{i+1 / 2, j} \notin(0,1), \\ (2-\theta) u_{i+1 / 2, j}-(1-\theta) u_{i+3 / 2, j} & \text { se } 0 \leq \hat{u}_{i+1 / 2, j}<a, \\ \alpha_{U} u_{i+1 / 2, j}+\alpha_{D} u_{i-1 / 2, j}+\alpha_{R} u_{i+3 / 2, j} & \text { se } a \leq \hat{u}_{i+1 / 2, j} \leq b, \\ (1-\theta) u_{i-1 / 2, j}+\theta u_{i+1 / 2, j} & \text { se } b<\hat{u}_{i+1 / 2, j} \leq 1 .\end{cases}
$$

Considera-se, agora, a variável $\phi=c$ transportada com velocidade $u$ na direção $z$, no ponto $(i, j)$

$$
\left.\frac{\partial(u c)}{\partial z}\right|_{i, j} \approx \frac{(u c)_{i+1 / 2, j}-(u c)_{i-1 / 2, j}}{\Delta z}
$$


Aproximando-se os termos $c_{i+1 / 2, j}$ e $c_{i-1 / 2, j}$, têm-se:

- Para $u_{i+1 / 2, j}>0$

$$
D=(i+1, j), U=(i, j), \quad R=(i-1, j), \hat{c}_{i, j}=\frac{c_{i, j}-c_{i-1, j}}{c_{i+1, j}-c_{i-1, j}}
$$

\section{WACEB}

$$
c_{i+1 / 2, j}= \begin{cases}c_{i, j} & \text { se } \hat{c}_{i, j} \notin[0,1], \\ 2 c_{i, j}-c_{i-1, j} & \text { se } 0 \leq \hat{c}_{i, j}<3 / 10, \\ \frac{1}{8}\left(3 c_{i+1, j}+6 c_{i, j}-c_{i-1, j}\right) & \text { se } 3 / 10 \leq \hat{c}_{i, j} \leq 5 / 6, \\ c_{i+1, j} & \text { se } 5 / 6<\hat{c}_{i, j} \leq 1,\end{cases}
$$

CUBISTA

$$
c_{i+1 / 2, j}= \begin{cases}c_{i, j} & \text { se } \hat{c}_{i, j} \notin[0,1], \\ \frac{1}{4}\left(7 c_{i, j}-3 c_{i-1, j}\right) & \text { se } 0 \leq \hat{c}_{i, j}<3 / 8, \\ \frac{1}{8}\left(3 c_{i+1, j}+6 c_{i, j}-c_{i-1, j}\right) & \text { se } 3 / 8 \leq \hat{c}_{i, j} \leq 3 / 4, \\ \frac{1}{4}\left(3 c_{i+1, j}+c_{i, j}\right) & \text { se } 3 / 4<\hat{c}_{i, j} \leq 1,\end{cases}
$$

QUICKEST adaptativo

$$
c_{i+1 / 2, j}= \begin{cases}c_{i, j} & \text { se } \hat{c}_{i, j} \notin(0,1), \\ (2-\theta) c_{i, j}-(1-\theta) c_{i-1, j} & \text { se } 0 \leq \hat{c}_{i, j}<a, \\ \alpha_{U} c_{i, j}+\alpha_{D} c_{i+1, j}+\alpha_{R} c_{i-1, j} & \text { se } a \leq \hat{c}_{i, j} \leq b, \\ (1-\theta) c_{i+1, j}+\theta c_{i, j} & \text { se } b<\hat{c}_{i, j} \leq 1,\end{cases}
$$


- Para $u_{i+1 / 2, j}<0$

$$
D=(i, j), U=(i+1, j), \quad R=(i+2, j), \hat{c}_{i+1, j}=\frac{c_{i+1, j}-c_{i+2, j}}{c_{i, j}-c_{i+2, j}}
$$

WACEB

$$
c_{i+1 / 2, j}= \begin{cases}c_{i+1, j} & \text { se } \hat{c}_{i+1, j} \notin[0,1], \\ 2 c_{i+1, j}-c_{i+2, j} & \text { se } 0 \leq \hat{c}_{i+1, j}<3 / 10, \\ \frac{1}{8}\left(3 c_{i, j}+6 c_{i+1, j}-c_{i+2, j}\right) & \text { se } 3 / 10 \leq \hat{c}_{i+1, j} \leq 5 / 6, \\ c_{i, j} & \text { se } 5 / 6<\hat{c}_{i+1, j} \leq 1,\end{cases}
$$

CUBISTA

$$
c_{i+1 / 2, j}= \begin{cases}c_{i+1, j} & \text { se } \hat{c}_{i+1, j} \notin[0,1], \\ \frac{1}{4}\left(7 c_{i+1, j}-3 c_{i+2, j}\right) & \text { se } 0 \leq \hat{c}_{i+1, j}<3 / 8, \\ \frac{1}{8}\left(3 c_{i, j}+6 c_{i+1, j}-c_{i+2, j}\right) & \text { se } 3 / 8 \leq \hat{c}_{i+1, j} \leq 3 / 4, \\ \frac{1}{4}\left(3 c_{i, j}+c_{i+1, j}\right) & \text { se } 3 / 4<\hat{c}_{i+1, j} \leq 1,\end{cases}
$$

QUICKEST adaptativo

$$
c_{i+1 / 2, j}= \begin{cases}c_{i+1, j} & \text { se } \hat{c}_{i+1, j} \notin(0,1), \\ (2-\theta) c_{i+1, j}-(1-\theta) c_{i+2, j} & \text { se } 0 \leq \hat{c}_{i+1, j}<a, \\ \alpha_{U} c_{i+1, j}+\alpha_{D} c_{i, j}+\alpha_{R} c_{i+2, j} & \text { se } a \leq \hat{c}_{i+1, j} \leq b, \\ (1-\theta) c_{i, j}+\theta c_{i+1, j} & \text { se } b<\hat{c}_{i+1, j} \leq 1,\end{cases}
$$


- Para $u_{i-1 / 2, j}>0$

$$
D=(i, j), U=(i-1, j), \quad R=(i-2, j), \hat{c}_{i-1, j}=\frac{c_{i-1, j}-c_{i-2, j}}{c_{i, j}-c_{i-2, j}}
$$

WACEB

$$
c_{i-1 / 2, j}= \begin{cases}c_{i-1, j} & \text { se } \hat{c}_{i-1, j} \notin[0,1], \\ 2 c_{i-1, j}-c_{i-2, j} & \text { se } 0 \leq \hat{c}_{i-1, j}<3 / 10, \\ \frac{1}{8}\left(3 c_{i, j}+6 c_{i-1, j}-c_{i-2, j}\right) & \text { se } 3 / 10 \leq \hat{c}_{i-1, j} \leq 5 / 6, \\ c_{i, j} & \text { se } 5 / 6<\hat{c}_{i-1, j} \leq 1,\end{cases}
$$

CUBISTA

$$
c_{i-1 / 2, j}= \begin{cases}c_{i-1, j} & \text { se } \hat{c}_{i-1, j} \notin[0,1], \\ \frac{1}{4}\left(7 c_{i-1, j}-3 c_{i-2, j}\right) & \text { se } 0 \leq \hat{c}_{i-1, j}<3 / 8, \\ \frac{1}{8}\left(3 c_{i, j}+6 c_{i-1, j}-c_{i-2, j}\right) & \text { se } 3 / 8 \leq \hat{c}_{i-1, j} \leq 3 / 4, \\ \frac{1}{4}\left(3 c_{i, j}+c_{i-1, j}\right) & \text { se } 3 / 4<\hat{c}_{i-1, j} \leq 1,\end{cases}
$$

QUICKEST adaptativo

$$
c_{i-1 / 2, j}= \begin{cases}c_{i-1, j} & \text { se } \hat{c}_{i-1, j} \notin(0,1), \\ (2-\theta) c_{i-1, j}-(1-\theta) c_{i-2, j} & \text { se } 0 \leq \hat{c}_{i-1, j}<a, \\ \alpha_{U} c_{i-1, j}+\alpha_{D} c_{i, j}+\alpha_{R} c_{i-2, j} & \text { se } a \leq \hat{c}_{i-1, j} \leq b, \\ (1-\theta) c_{i, j}+\theta c_{i-1, j} & \text { se } b<\hat{c}_{i-1, j} \leq 1,\end{cases}
$$


- Para $u_{i-1 / 2, j}<0$

$$
D=(i-1, j), U=(i, j), \quad R=(i+1, j), \quad \hat{c}_{i, j}=\frac{c_{i, j}-c_{i+1, j}}{c_{i-1, j}-c_{i+1, j}}
$$

WACEB

$$
c_{i-1 / 2, j}= \begin{cases}c_{i, j} & \text { se } \hat{c}_{i, j} \notin[0,1], \\ 2 c_{i, j}-c_{i+1, j} & \text { se } 0 \leq \hat{c}_{i, j}<3 / 10, \\ \frac{1}{8}\left(3 c_{i-1, j}+6 c_{i, j}-c_{i+1, j}\right) & \text { se } 3 / 10 \leq \hat{c}_{i, j} \leq 5 / 6, \\ c_{i-1, j} & \text { se } 5 / 6<\hat{c}_{i, j} \leq 1,\end{cases}
$$

CUBISTA

$$
c_{i-1 / 2, j}= \begin{cases}c_{i, j} & \text { se } \hat{c}_{i, j} \notin[0,1], \\ \frac{1}{4}\left(7 c_{i, j}-3 c_{i+1, j}\right) & \text { se } 0 \leq \hat{c}_{i, j}<3 / 8, \\ \frac{1}{8}\left(3 c_{i-1, j}+6 c_{i, j}-c_{i+1, j}\right) & \text { se } 3 / 8 \leq \hat{c}_{i, j} \leq 3 / 4, \\ \frac{1}{4}\left(3 c_{i-1, j}+c_{i, j}\right) & \text { se } 3 / 4<\hat{c}_{i, j} \leq 1,\end{cases}
$$

QUICKEST adaptativo

$$
c_{i-1 / 2, j}= \begin{cases}c_{i, j} & \text { se } \hat{c}_{i, j} \notin(0,1), \\ (2-\theta) c_{i, j}-(1-\theta) c_{i+1, j} & \text { se } 0 \leq \hat{c}_{i, j}<a, \\ \alpha_{U} c_{i, j}+\alpha_{D} c_{i-1, j}+\alpha_{R} c_{i+1, j} & \text { se } a \leq \hat{c}_{i, j} \leq b, \\ (1-\theta) c_{i-1, j}+\theta c_{i, j} & \text { se } b<\hat{c}_{i, j} \leq 1 .\end{cases}
$$


Da mesma forma como os termos não-lineares (4.1.18) e (4.1.19) foram aproximados pelos esquemas de discretização, todas as outras derivadas convectivas nas equações de conservação também foram discretizadas.

Uma observação importante a ser feita é o fato de que os esquemas utilizam três pontos vizinhos $(D, U$ e $R$ ) para aproximar as propriedades. Assim, em células próximas às fronteiras, a discretização pode solicitar valores fora do domínio computacional, como, por exemplo, para o caso da aproximação de $u_{i, j}$ em células adjacentes à fronteira de entrada, quando $\bar{u}_{i, j}>0$ deve-se ter definido o valor de $R=(i-3 / 2, j)$. Nesse caso, e em casos semelhantes, um esquema de primeira ordem deve ser empregado. Para este trabalho, utilizou-se o esquema upwind:

$$
\phi_{f}=\phi_{U} \quad \text { ou } \quad u_{i, j}=u_{i-1 / 2, j} \quad \text { para } \quad \bar{u}_{i, j}>0
$$

\subsection{Método numérico}

Como já comentado anteriormente, o método Sola ([HIRT et al., 1975]) é conceitualmente semelhante ao método MAC. A diferença entre os dois está basicamente na forma de resolução da equação de Poisson. Em vez de calcular diretamente a pressão no nível de tempo $N+1$ em uma célula, como no MAC, ele (Sola) utiliza um procedimento iterativo de cálculo de correções $\delta p$ para a pressão:

$$
\delta p_{i, j}=\frac{-\omega D_{i, j}^{N+1}}{2 \Delta t\left(\frac{1}{(\Delta z)^{2}}+\frac{1}{(\Delta r)^{2}}\right)}
$$

em que $\omega(1<\omega<2)$ é um fato de relaxação que pode acelerar a convergência desse 
processo iterativo e $D_{i, j}^{N+1}$ a dilatação $D_{i, j}=\left.\frac{\partial u}{\partial z}\right|_{i, j}+\left.\frac{1}{r} \frac{\partial(r v)}{\partial r}\right|_{i, j}$.

\section{Ciclo computacional}

Para a simulação dos efeitos do escoamento em tubos porosos, admite-se que, em um dado $t_{0}$, as variáveis dependentes são conhecidas e as condições de fronteira associadas estão definidas. Assim, as etapas do ciclo computacional são divididas em cinco passos:

- passo 1: calculam-se os valores de $c_{i, j}^{N+1}$ dados por (4.1.7);

- passo 2: a partir dos valores $u, v$ e $p$ no nível de tempo $N$, calculam-se os valores de $u_{i+1 / 2, j}^{N+1}$ e $v_{i, j+1 / 2}^{N+1}$ dados por (4.1.5) e (4.1.6), respectivamente, para todos os pontos internos;

- passo 3: para cada célula $(i, j)$

(i) calcula-se a correção para pressão definida pela expressão (4.2.1);

(ii) determina-se $p_{i, j}$, utilizando-se

$$
p_{i, j}^{n+1,(k+1)}=p_{i, j}^{n+1,(k)}+\delta p_{i, j}^{(k)}
$$

em que $k$ é o número de iterações e

(iii) corrigem-se as velocidades nas faces das células por meio das expressões

$$
\begin{gathered}
u_{i+1 / 2, j}^{n+1,(k+1 / 2)}=u_{i+1 / 2, j}^{n+1,(k)}+\Delta t \frac{\delta p_{i, j}^{(k)}}{\Delta z} \\
u_{i-1 / 2, j}^{n+1,(k+1)}=u_{i-1 / 2, j}^{n+1,(k)}-\Delta t \frac{\delta p_{i, j}^{(k)}}{\Delta z}
\end{gathered}
$$




$$
\begin{gathered}
v_{i, j+1 / 2}^{n+1,(k+1 / 2)}=v_{i, j+1 / 2}^{n+1,(k)}+\Delta t \frac{\delta p_{i, j}^{(k)}}{\Delta r} \\
v_{i, j-1 / 2}^{n+1,(k+1)}=v_{i, j-1 / 2}^{n+1,(k)}-\Delta t \frac{\delta p_{i, j}^{(k)}}{\Delta r}
\end{gathered}
$$

As velocidades que são condições de fronteira não são corrigidas;

- passo 4: repete-se o passo 3 até que

$$
\max \left|D_{i, j}^{(k)}\right| \leq \epsilon
$$

A condição de parada (4.2.7) garante que o módulo da dilatação máxima nas células do domínio não seja maior que $\epsilon$, cujo valor depende do problema tratado. Pode-se calcular $\epsilon$ por meio de uma expressão do tipo (|FORTUNA, 2000|)

$$
\epsilon=\frac{\sigma_{c}}{N_{c}}
$$

em que $\sigma_{c}$, cujo valor está no intervalo entre $10^{-4}$ e $10^{-7}$, é um parâmetro ajustável, e $N_{c}$ é o número de células em que se calcula a dilatação $D_{i, j}$. O parâmetro $\sigma_{c}$ deve ser tal que a solução numérica indenpenda de valores de $\sigma_{c}$ menores que o escolhido. Com essa condição satisfeita, as velocidades $u$ e $v$ e a pressão $p$ estão no nível de tempo $t=t_{0}+\Delta t \mathrm{e}$

- passo 5: tomando-se adequado valor para $\Delta t$, retorna-se ao passo 1 até que

$$
\frac{1}{\Delta t}\left(\sum_{i=1} \sum_{j=1}\left|u_{i, j}^{n+1}-u_{i, j}^{n}\right|+\left|v_{i, j}^{n+1}-v_{i, j}^{n}\right|\right) \leq \varepsilon_{v} \quad \mathrm{e}
$$




$$
\frac{1}{\Delta t}\left(\sum_{i=1} \sum_{j=1}\left|c_{i, j}^{n+1}-c_{i, j}^{n}\right|\right) \leq \varepsilon_{c} .
$$

Os parâmetros $\varepsilon_{v}$ e $\varepsilon_{c}$ também dependem do problema que será calculado por uma expressão análoga a (4.2.8), com $10^{-3} \leq \sigma_{c} \leq 10^{-5}$.

\subsection{Controle do passo no tempo}

A forma explícita do cálculo de $u, v$ e $c$ impõe restrições severas aos valores permitidos de $\Delta t$. A cada ciclo, o tamanho do passo no tempo é obtido segundo as restrições ([FERREIRA, 2001]):

(i) o fluido não pode percorrer uma distância maior que o comprimento de uma célula a cada passo no tempo. Assim, o valor de $\Delta t$ deve satisfazer às restrições

$$
\Delta t_{1}<\frac{\Delta z}{|u|_{\max }} \quad \text { e } \quad \Delta t_{2}<\frac{\Delta r}{|v|_{\max }}
$$

em que $u_{\max }$ e $v_{\max }$ são os valores máximos dos adimensionais $u$ e $v$, respectivamente;

(ii) a segunda restrição de estabilidade é devido à discretização das equações de conservação da quantidade de movimento

$$
\Delta t_{3}<\frac{R e}{16}\left(\frac{3 n+1}{n}\right)^{n}\left[\frac{(\Delta z)^{2}(\Delta r)^{2}}{(\Delta z)^{2}+(\Delta r)^{2}}\right]
$$

$\mathrm{e}$ 
(iii) análoga à restrição (ii), a terceira condição é devido à discretização explícita da equação de conservação das espécies

$$
\Delta t_{4}<\frac{P e}{4}\left[\frac{(\Delta z)^{2}(\Delta r)^{2}}{(\Delta z)^{2}+(\Delta z)^{2}}\right]
$$

Assim, o valor $\Delta t$ adotado deve satisfazer simultaneamente as condições (i), (ii) e (iii):

$$
\Delta t=A_{0} \min \left\{\Delta t_{1}, \Delta t_{2}, \Delta t_{3}, \Delta t_{4}\right\}
$$

em que a constante $0<A_{0} \leq 1$ é introduzida para manter os cálculos dentro de regiões estáveis. 


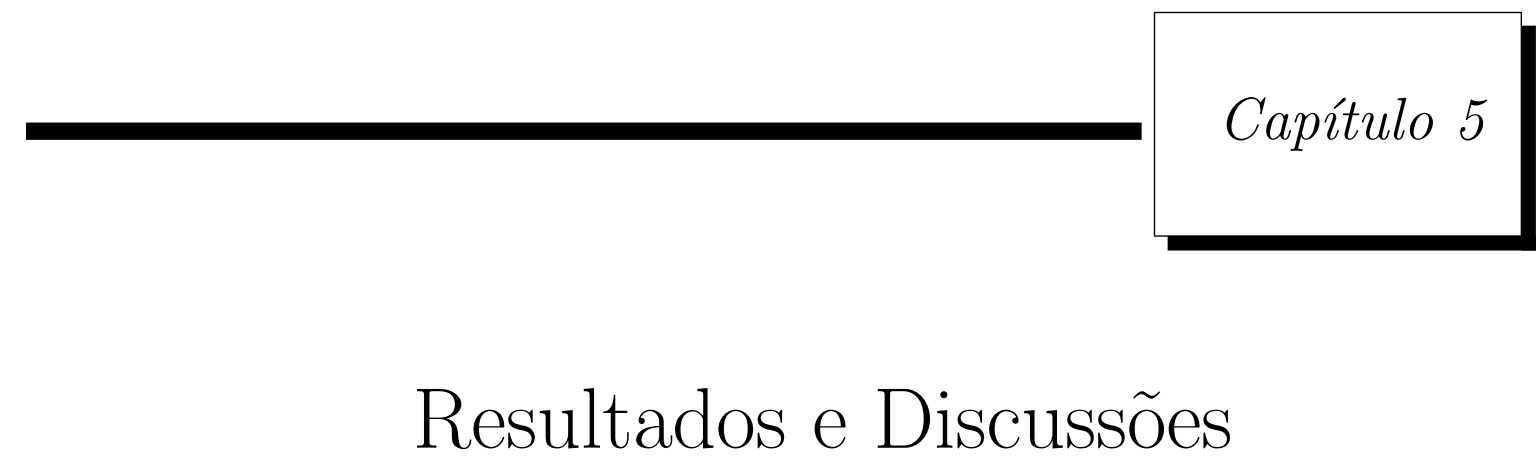

A maneira usual de validar modelos matemáticos, como deste trabalho, é promover a comparação dos resultados numéricos com resultados experimentais compatíveis com o problema físico. Porém, determinar experimentalmente algumas grandezas físicas não é uma tarefa simples e pode requerer construções experimentais de alto custo. Problemas como o desse estudo necessitam de tecnologias ainda não bem estabelecidas. Conseqüentemente, um conjunto adequado de resultados experimentais que possa contribuir para comparações com modelagens matemáticas não é encontrado na literatura, especialmente para problemas de transferência de massa com membranas e/ou em tubos permeáveis, em que a determinação física de grandezas essenciais, como a concentração no campo de escoamento, deve requerer sensores não intrusivos para medidas da concentração.

Uma forma de promover a validação da modelagem é comparar os resultados numéricos com resultados experimentais para a velocidade de permeação média em função da pressão transmembrana e do número de Reynolds, além de comparar com resultados de soluções analíticas da literatura como o número de Sherwood médio e local.

Neste capítulo, o modelo matemático apresentado no capítulo 3 é analisado em 
função das variações dos esquemas de discretização dos termos convectivos, os quais foram definidos no capítulo anterior. Como resultado dessa análise, um dos esquemas é selecionado para a modelagem do escoamento em tubos permeáveis e/ou em membranas tubulares. Também são apresentados os resultados dessa modelagem comparados com os da literatura, incluindo-se resultados experimentais, de soluções analíticas aproximadas e resultados experimentais gerados exclusivamente para este estudo.

\subsection{Análise dos esquemas convectivos}

Esta seção é reservada à análise dos resultados numéricos originários da implementação dos esquemas de discretização dos termos convectivos descritos no capítulo 4. Essa análise permitiu verificar qual o melhor esquema a ser implementado na modelagem do problema estudado. Para tanto, estudaram-se várias simulações com diversas condições de fronteira.

\subsubsection{Escoamento em tubos com paredes impermeáveis}

Nessa primeira análise, implementou-se um dos modelos mais simplificados para escoamento de fluidos: o escoamento de fluidos newtonianos em tubos impermeáveis para o regime laminar. Os resultados foram comparados com a solução analítica de Sparrow e Lin (1964). Os dados das simulações representadas nas Figs. 5.1 e 5.2 são os seguintes:

- diâmetro: $d=0,03 m$;

- número de Reynolds: $R e=100$;

- pressão inicial: $P_{0}=2 \times 10^{5} \mathrm{~Pa}$;

- viscosidade dinâmica: $\mu=0,001 \mathrm{Pas}^{-1}$; 
- massa específica: $\rho=1000 \mathrm{kgm}^{-3}$;

- índice de comportamento: $n=1,0$.
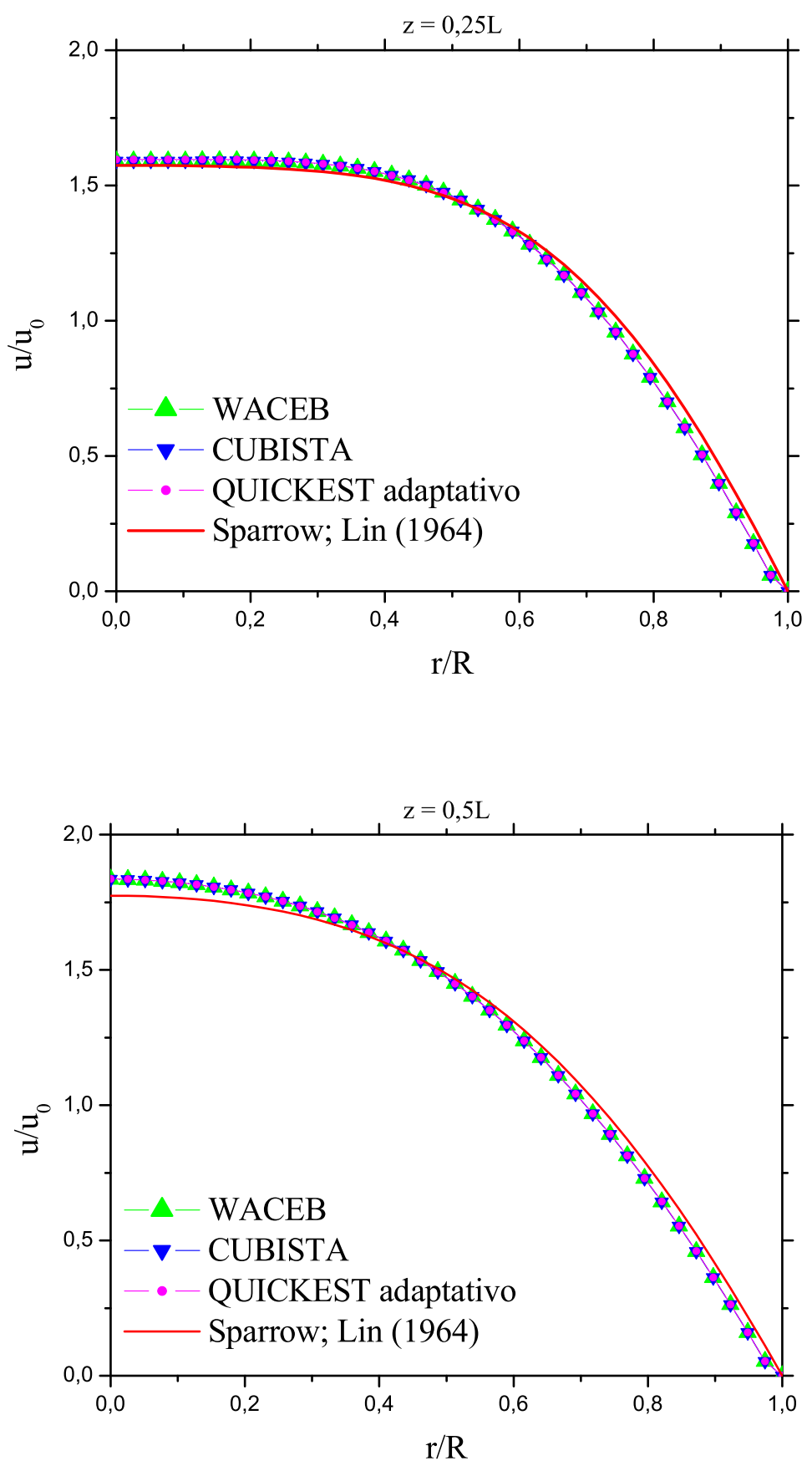

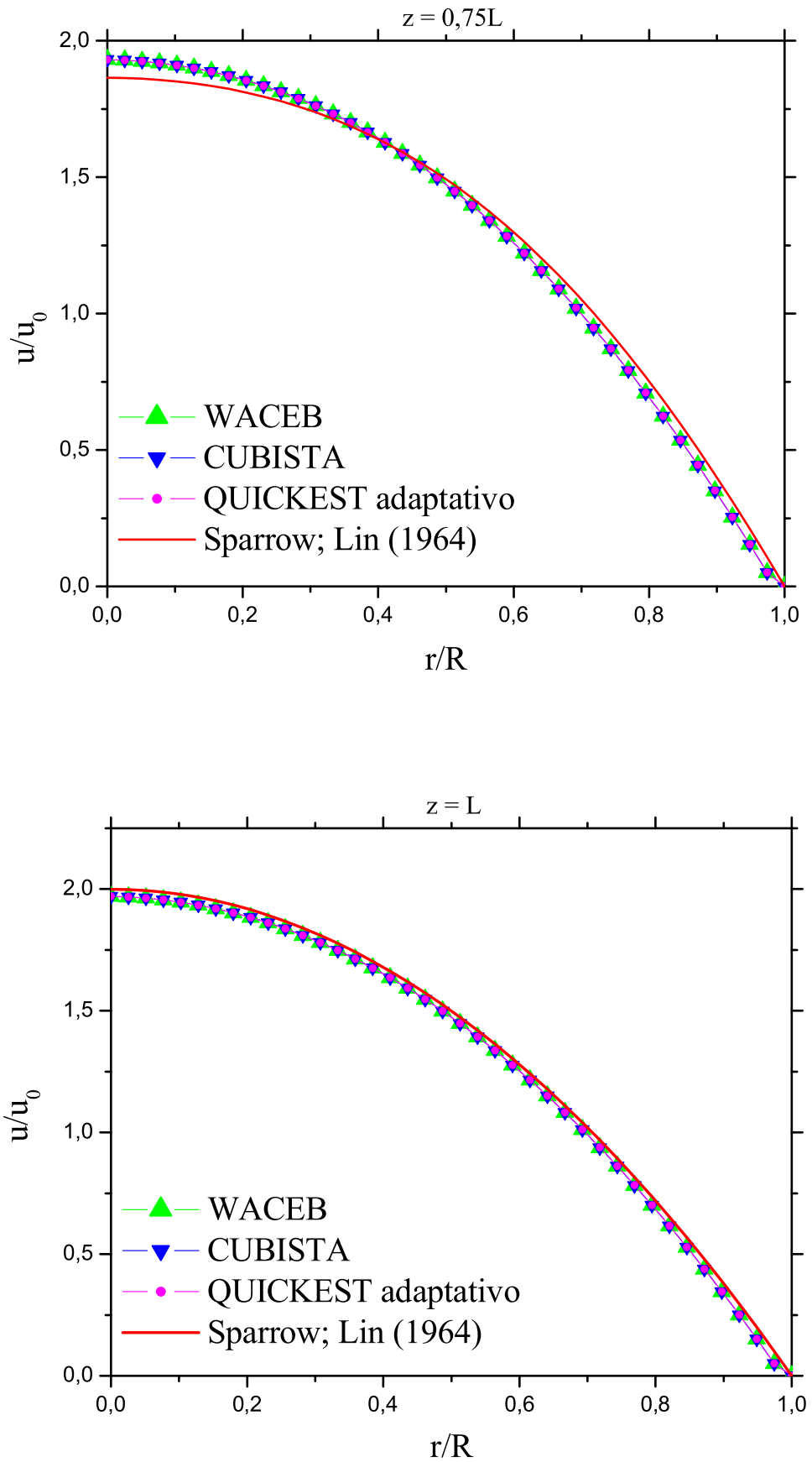

Figura 5.1: Perfil de velociadade axial em função da coordenada radial para os diferentes esquemas convectivos com uma malha computacional de dimensões 400x40.

Na Fig. 5.1, é apresentada uma comparação com Sparrow e Lin (1964) do perfil de velocidade na direção axial, $u / u_{0}$, como função da coordenada radial, $r / R$, (em 
$z / R=0,25 L / R, z / R=0,5 L / R, z / R=0,75 L / R$ e $z / R=L / R)$, para os diferentes esquemas convectivos.
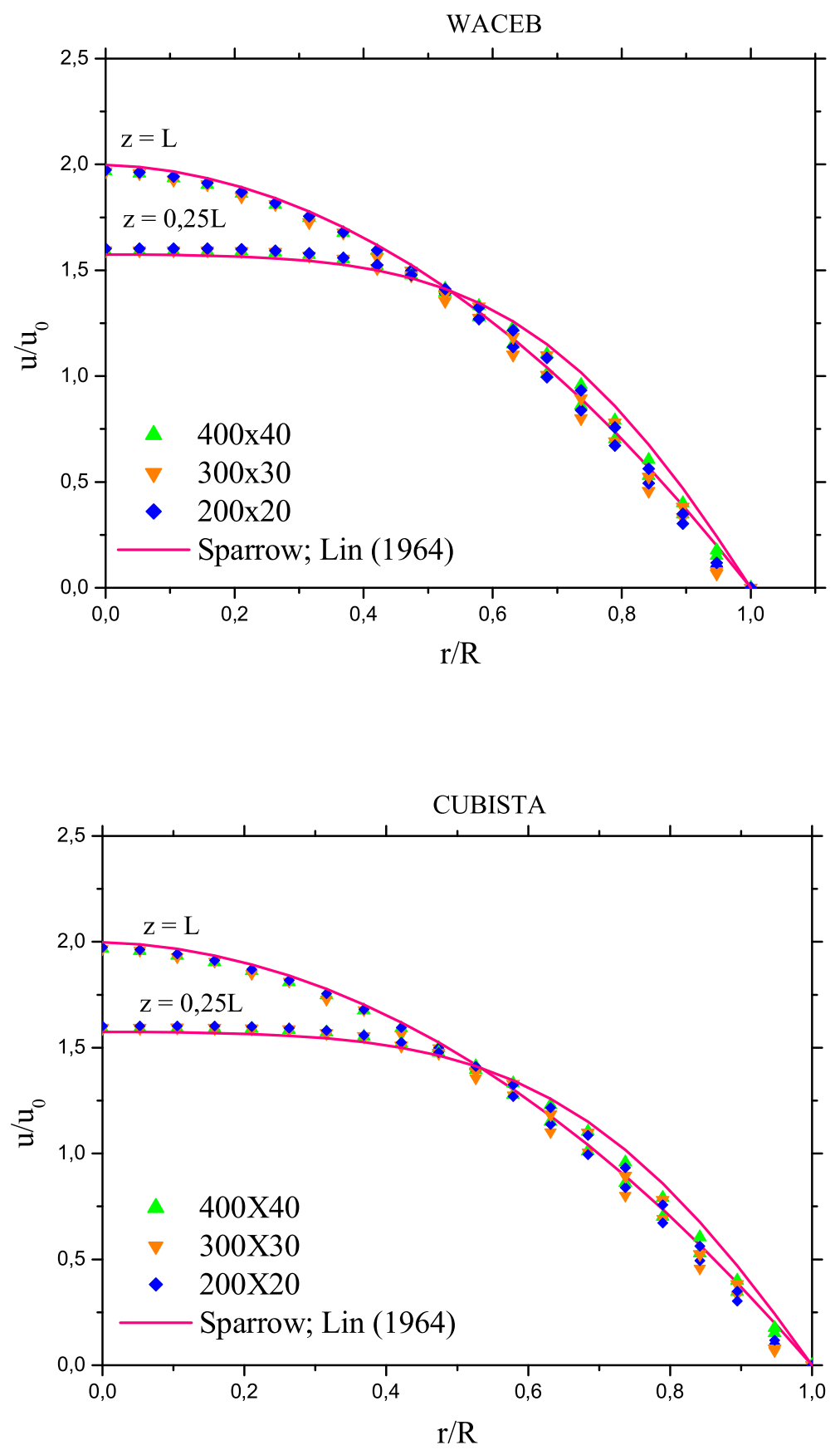


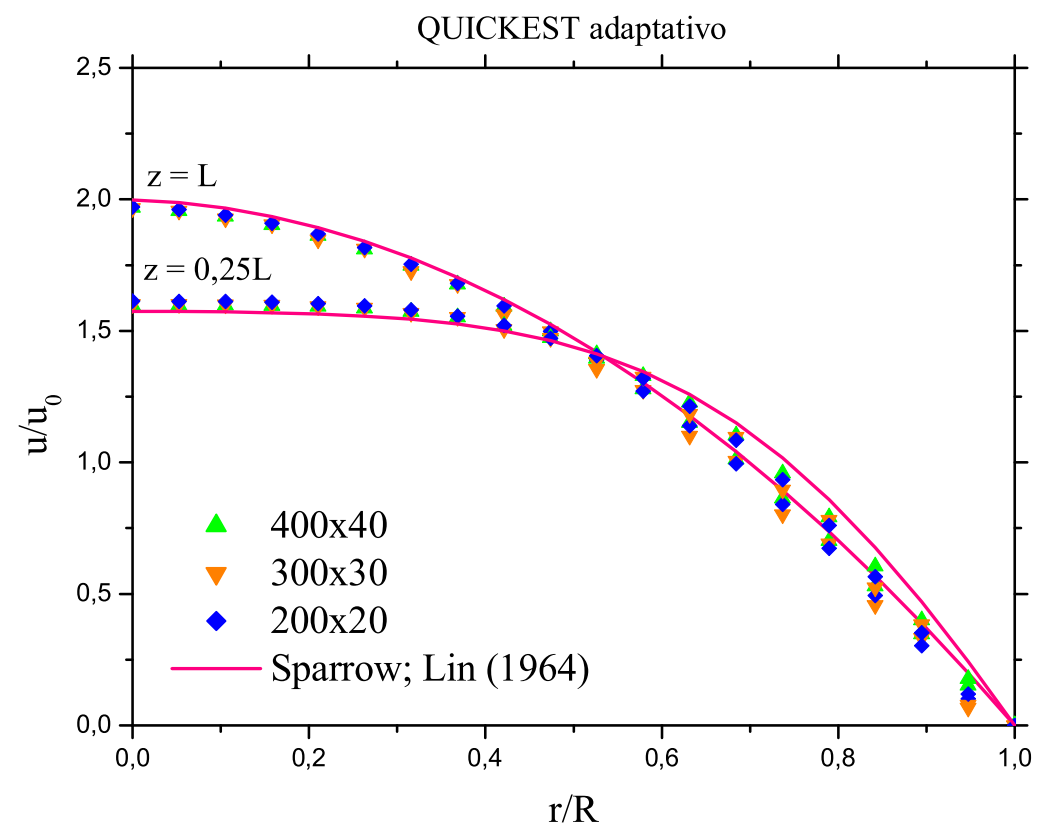

Figura 5.2: Perfil de velocidade axial em função da coordenada radial para diferentes malhas computacionais.

A Fig. 5.2 também apresenta resultados para os perfis de velocidade em função da coordenada radial, $r / R$, porém para diferentes malhas computacionais, em dois pontos diferentes do tubo $(z / R=L / R$ e $z / R=0,25 L / R)$, para os diferentes esquemas convectivos.

Nota-se nas Figs. 5.1 e 5.2 que os resultados numéricos produzidos durante a utilização dos esquemas WACEB, CUBISTA e QUICKEST adaptativo são praticamente coincidentes, não se apresentando variações para as diferentes malhas computacionais. Portanto, pode-se concluir que para o escoamento de entrada em regime laminar em tubos com paredes impermeáveis, os esquemas convectivos possuem um desempenho semelhante. 


\subsubsection{Escoamento em tubos com paredes permeáveis}

Nas próximas simulações, investiga-se o escoamento em tubos com paredes permeáveis, nas quais, devido ao gradiente de pressão, considera-se a transferência através da superfície do tubo. As velocidades de transferência na superfície tubular permeável são representadas de duas formas:

(i) na simulação do escoamento de água pura, utiliza-se a equação constitutiva de Darcy (DAMAK et al., 2004c)

$$
v_{w}=\frac{\Delta p}{R_{m}}
$$

$\mathrm{e}$

(ii) nas demais simulações, emprega-se o modelo de resistência em série

$$
v_{w}=\frac{\Delta p}{R_{m}+R_{f}+\phi \Delta p}
$$

Os dados das simulações representadas na Fig. 5.3 são os seguintes:

- diâmetro: $d=0,03 m$;

- número de células computacionais: $70 \times 30$;

- número de Reynolds: $R e=100$;

- pressão inicial: $P_{0}=2 \times 10^{5} \mathrm{~Pa}$;

- viscosidade dinâmica: $\mu=0,001 \mathrm{Pas}^{-1}$;

- massa específica: $\rho=1000 \mathrm{kgm}^{-3}$.

Na Fig. 5.3, são apresentados os perfis da queda de pressão em função da coordenada $z /(R \times R e)$ comparados com os resultados da solução analítica de Karode (2001), 
para cada um dos esquemas convectivos estudados. Nessas simulações, utilizou-se a água pura como fluido, conseqüentemente a equação de Darcy (Eq. (5.1.1)) para definir a velocidade na parede permeável. Observa-se que os resultados, quando se implementaram os esquemas de discretização dos termos convectivos, são bastante parecidos e com uma boa concordância com a literatura para $z /(R \times R e)$ entre 0,04 e 0,06 , com erro relativd ${ }^{1}$ máximo de 3,088\%.

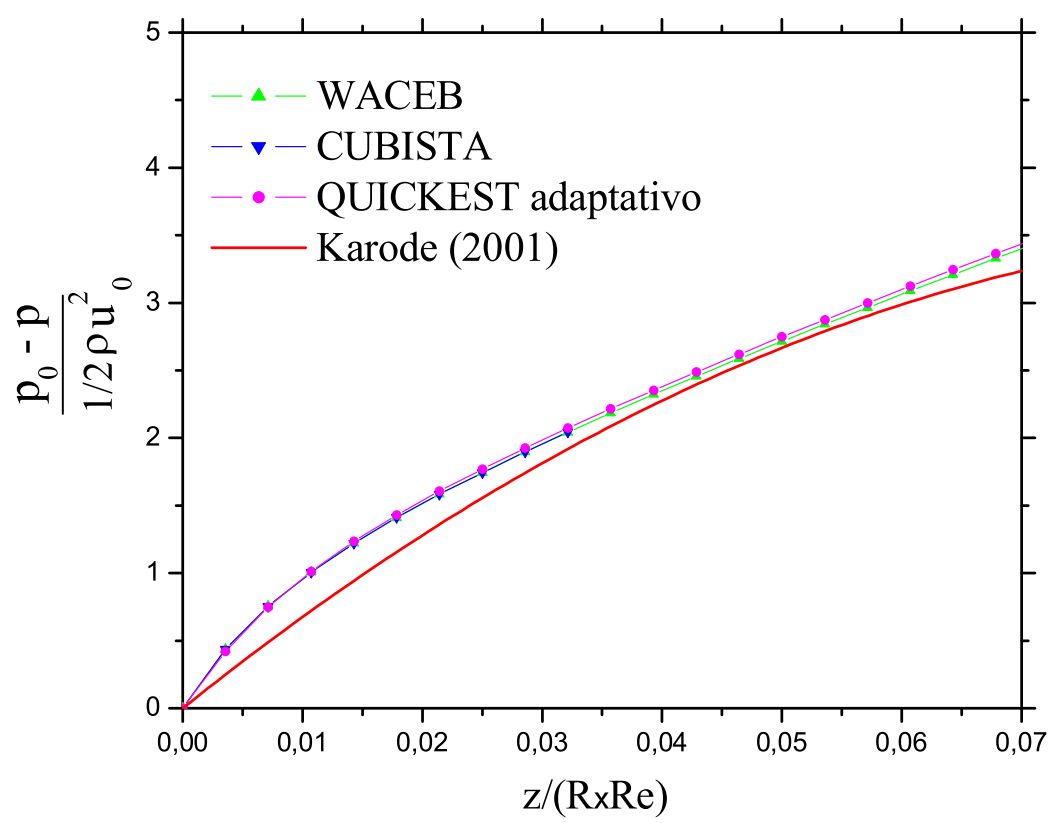

Figura 5.3: Perfil da queda de pressão em função da coordenada $z /(R \times R e)$.

Para as próximas simulações, as velocidades na parede permeável foram obtidas pela equação (5.1.2), pois nesse caso, o fluido é uma mistura ou solução que pode promover o fenômeno de polarização. Os dados das simulações representadas na Fig. 5.4 são os seguintes:

- diâmetro: $d=0,03 \mathrm{~m}$;

${ }^{1}$ Erro relativo: $E R \%=\left(\frac{\mid \text { Resultadoliteratura }_{\text {Resultado }} \text { Rumerico } \mid}{\text { Resultado }}\right) \cdot 100 \%$. 
- número de células computacionais: $70 \times 30$;

- número de Reynolds: $R e=100$;

- pressão inicial: $P_{0}=2 \times 10^{5} \mathrm{~Pa}$;

- coeficiente de consistência: $m=0,001$ Pas $^{n}$

- massa específica: $\rho=1000 \mathrm{kgm}^{-3}$

- coeficiente de difusão: $D=3 \times 10^{-8} \mathrm{~m}^{2} \mathrm{~s}^{-1}$;

- resistência da membrana: $R_{m}=0,25 \times 10^{10} \mathrm{kgm}^{-2} \mathrm{~s}^{-1}$;

- resistência devido à camada de concentração: $R_{f}=1,503 \times 10^{10} \mathrm{kgm}^{-2} \mathrm{~s}^{-1}$;

- constante de proporcionalidade: $\phi=1,986 \times 10^{5} \mathrm{sm}^{-1}$.

São apresentados na Fig. 5.4 resultados do adimensional número de Sherwood local em função da coordenada axial, $z / R$, para os diferentes esquemas convectivos e variáveis índice de comportamento, $n$, comparados com os resultados do trabalho de Ranjan et al. (2004). Observa-se que os resultados numéricos são assintóticos aos de Ranjan et al. (2004a) a partir de $z / R=0.8$, prevendo-se o mesmo valor do número de Sherwood na região desenvolvida.

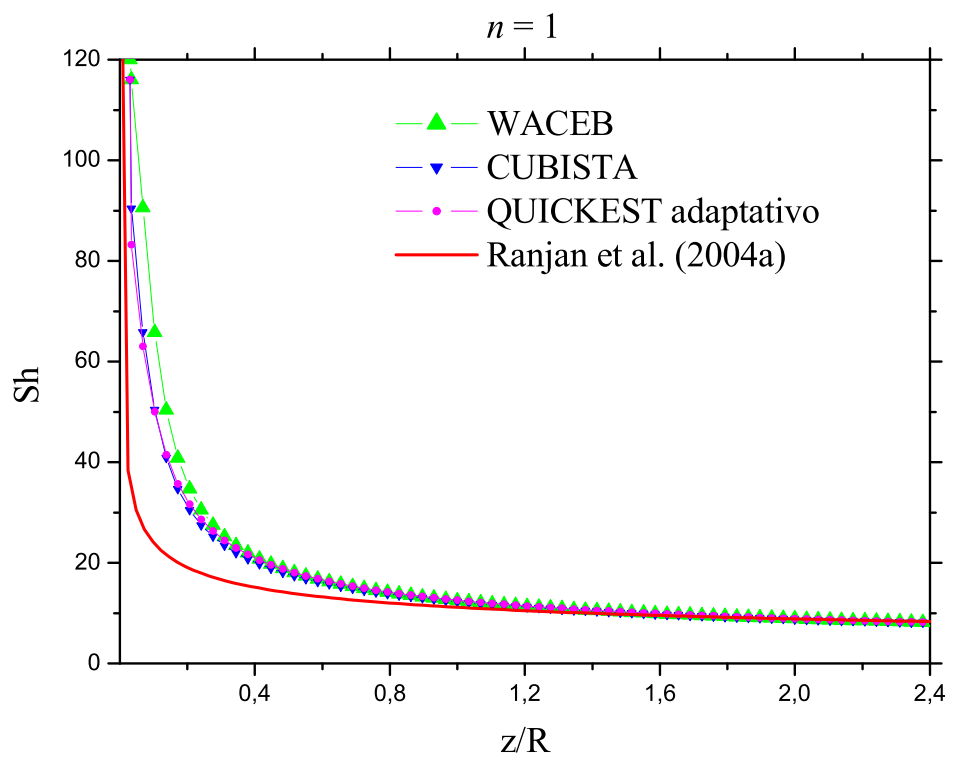



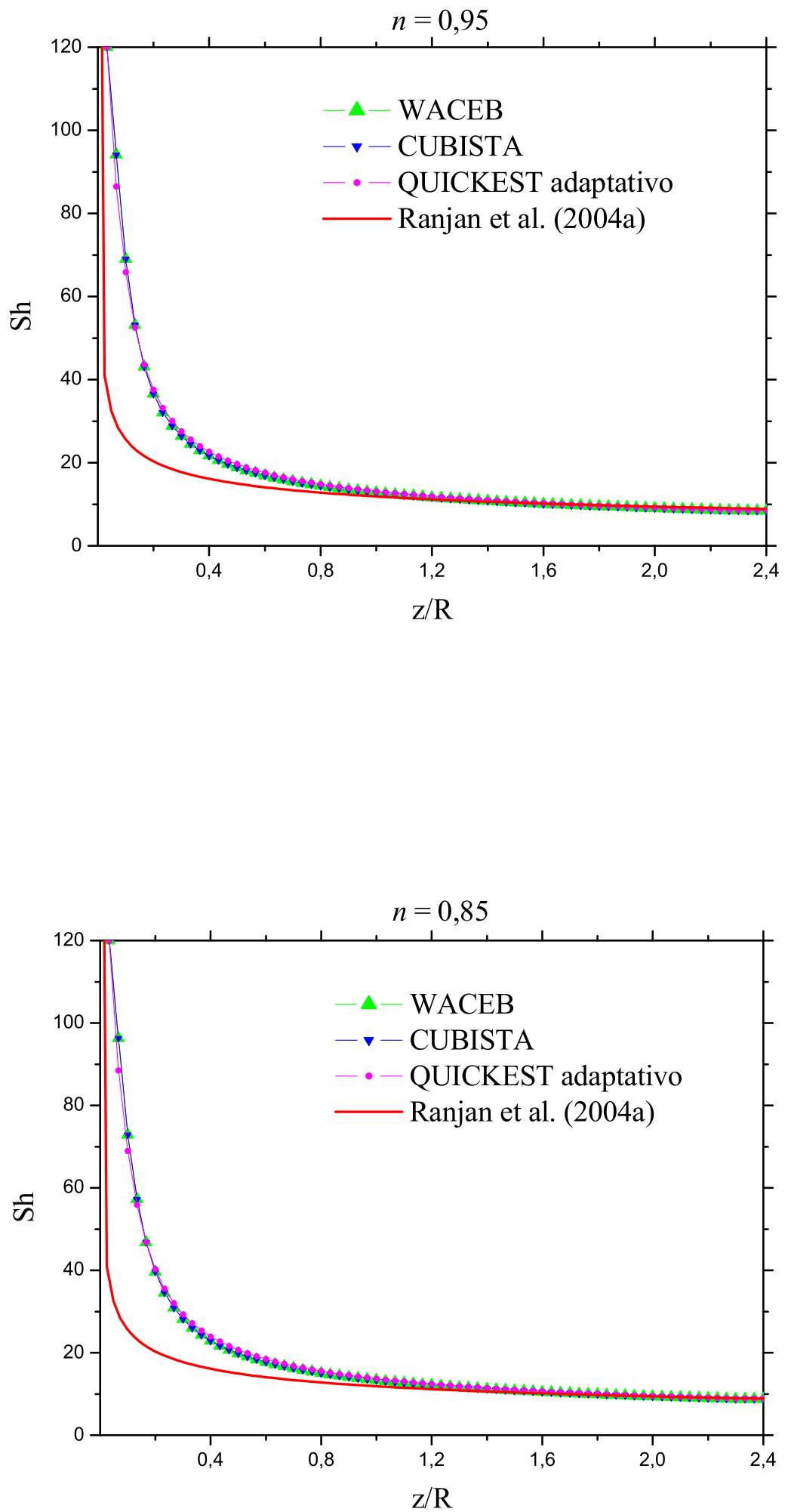


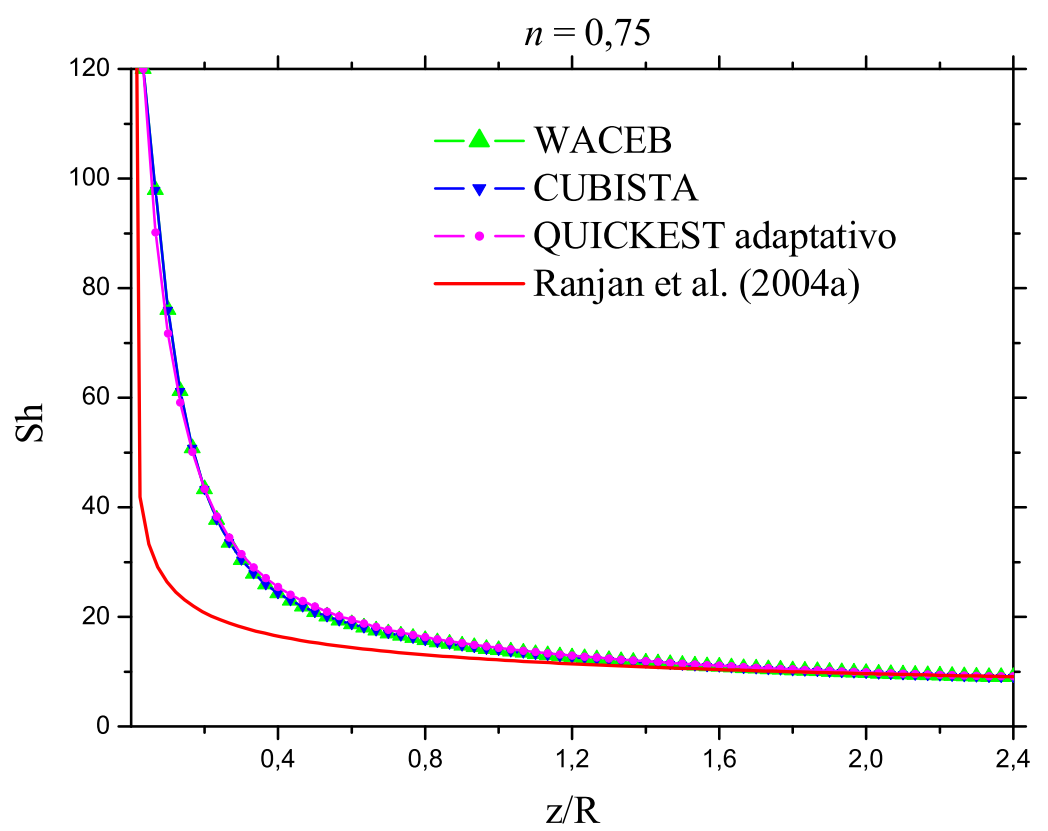

Figura 5.4: Número de Sherwood em função da coordenada axial.

Comparações dos resultados numéricos com os de uma solução analítica/numérica de Venezuela e Fontes (2007) são apresentadas na Fig. 5.5. Esses resultados são do perfil de concentração próximo à superfície permeável em função da coordenada axial, $z / R$, para os três esquemas convectivos. Observa-se a partir da Fig. 5.5 e da Tab. 5.1, a qual representa o erro relativo máximo dos esquema em relação a solução analítica/numérica, que o esquema QUICKEST adaptativo produziu resultados mais próximos da solução da literatura, com erro relativo menor em relação aos outros esquemas. Outra observação a ser considerada é a diferença dos resultados numéricos e analítico/numérico que acredita-se ser devido ao fato de na modelagem híbrida, a equação de conservação das espécies químicas ter uma formulação simplificada, ao contrário do presente modelo numérico que possui uma modelagem completa, o que justificaria a diferença entre os resultados. Entende-se que a mesma explicação justifique as diferenças observadas nas Figs. 5.4 e 5.6. Os dados das simulações representadas nas Figs. 5.5 e 5.6 são os seguintes: 
- diâmetro: $d=0,03 m$;

- número de células computacionais: $100 \times 40$;

- número de Reynolds: $R e=100$;

- pressão inicial: $P_{0}=2 \times 10^{5} P a$;

- coeficiente de consistência: $\mu=0,001$ Pas

- massa específica: $\rho=1000 \mathrm{kgm}^{-3}$

- coeficiente de difusão: $D=7 \times 10^{-8} \mathrm{~m}^{2} \mathrm{~s}^{-1}$;

- resistência da membrana: $R_{m}=0,25 \times 10^{9} \mathrm{kgm}^{-2} \mathrm{~s}^{-1}$;

- resistência devido à camada de concentração: $R_{f}=0,503 \times 10^{9} \mathrm{kgm}^{-2} \mathrm{~s}^{-1}$;

- constante de proporcionalidade: $\phi=0,986 \times 10^{5} \mathrm{sm}^{-1}$.

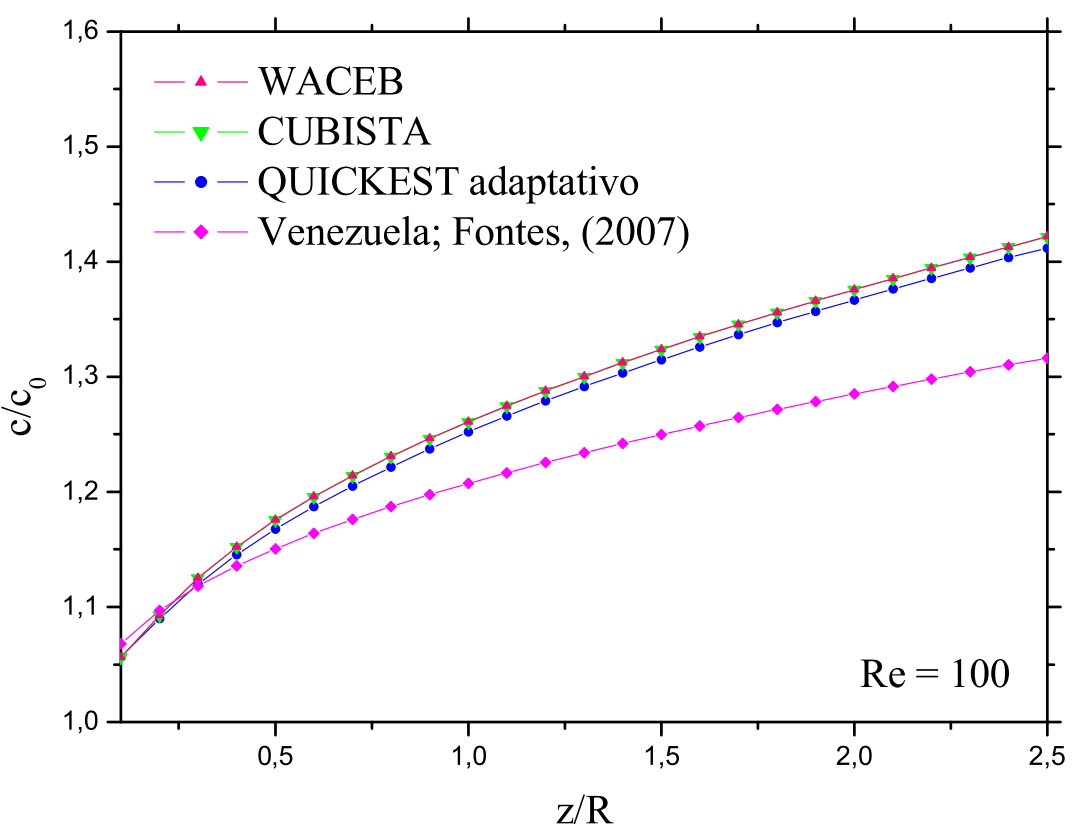




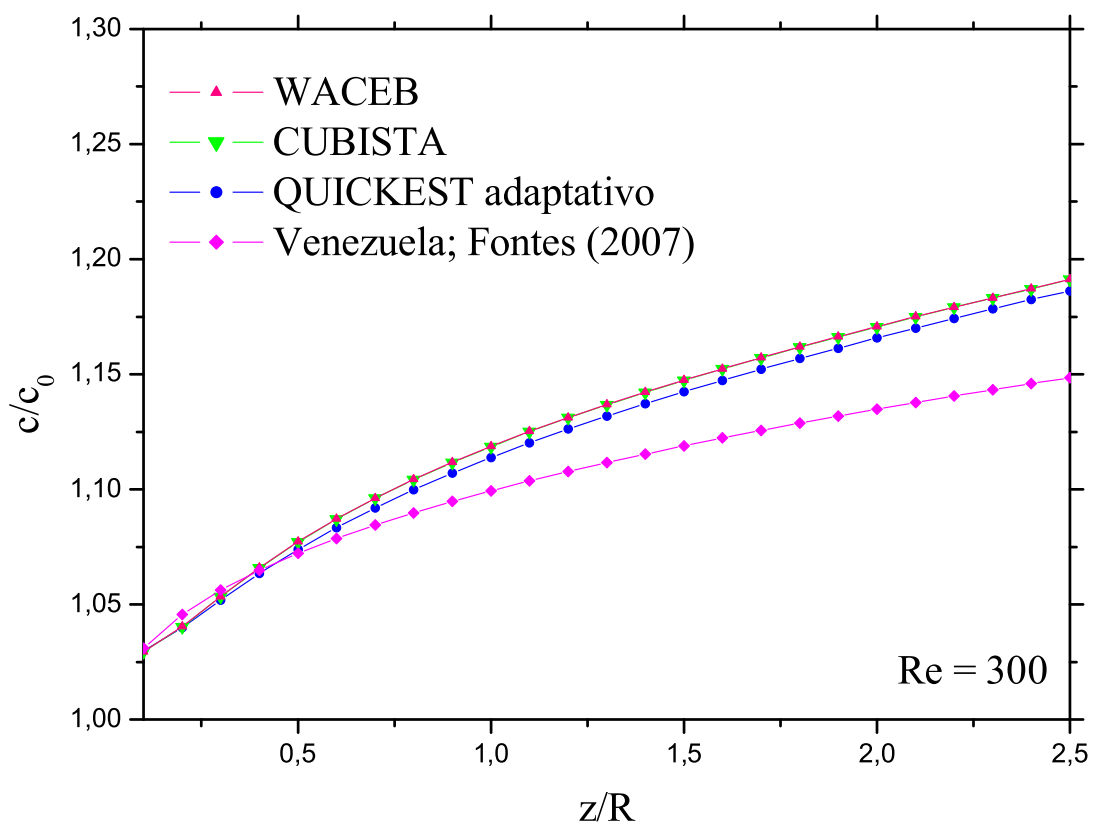

Figura 5.5: Concentração próxima à superfície permeável em função da coordenada axial, para números de Reynolds 100 e 300.

\begin{tabular}{|l||c|c|}
\hline \multicolumn{3}{|c|}{ ER\% } \\
\hline & $R e=100$ & $R e=300$ \\
\hline \hline WACEB & 8,02 & 3,72 \\
\hline CUBISTA & 8 & 3,71 \\
\hline QUICKEST adaptativo & 7,26 & 3,28 \\
\hline
\end{tabular}

Tabela 5.1: Erro relativo máximo referente às simulações representadas na Fig. 5.5.

Na Fig. 5.6, são mostradas comparações dos resultados numéricos, produzidos pelos esquemas convectivos, com resultados analíticos de Ranjan et al. (2004a), para número de Sherwood médio em função do índice de comportamento, $n$, com números de Reynolds 150 e 300. Novamente, observa-se que o esquema QUICKEST adaptativo produziu o melhor resultado, como pode-se verificar também nas Tabs. 5.2 e 5.3 , as quais representam o erro relativo dos esquemas em relação à solução analítica. 

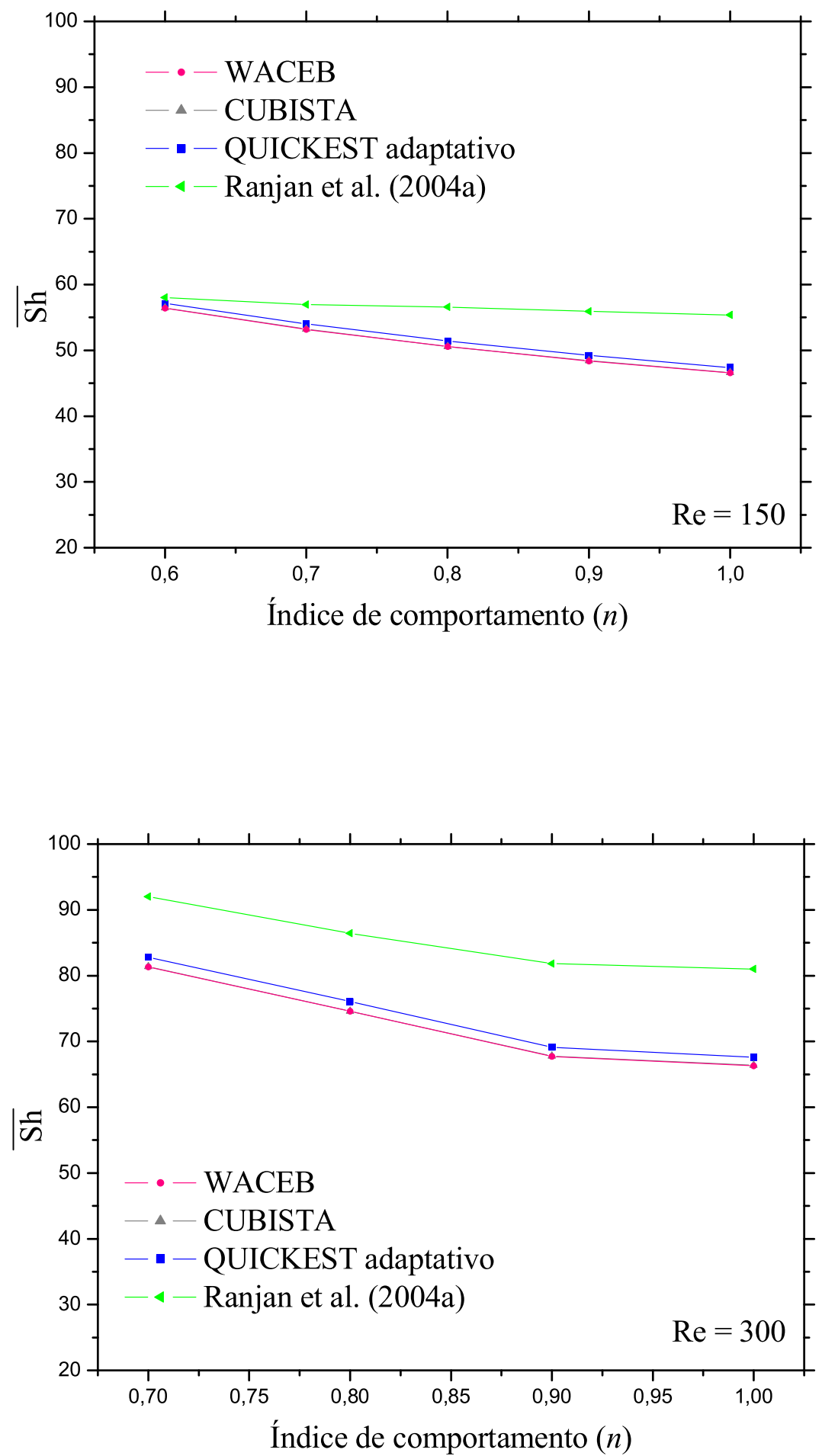

Figura 5.6: Número de Sherwood médio em função de $n$, para números de Reynolds 150 e 300 . 


\begin{tabular}{|l||c|c|c|}
\hline \multicolumn{3}{|c|}{ ER\% } \\
\hline$n$ & WACEB & CUBISTA & QUICKEST adaptativo \\
\hline \hline 0,6 & 2,75 & 2,7 & 1,53 \\
\hline 0,7 & 6,63 & 6,58 & 5,18 \\
\hline 0,8 & 10,66 & 10,61 & 9,14 \\
\hline 0,9 & 13,45 & 13,38 & 11,94 \\
\hline 1,0 & 15,86 & 15,76 & 14,38 \\
\hline
\end{tabular}

Tabela 5.2: Erro relativo referente às simulações representadas na Fig. 5.6 para $R e=150$.

\begin{tabular}{|l||c|c|c|}
\hline \multicolumn{3}{|c|}{ ER\% } \\
\hline$n$ & WACEB & CUBISTA & QUICKEST adaptativo \\
\hline \hline 0,7 & 11,6 & 11,55 & 10 \\
\hline 0,8 & 13,74 & 13,69 & 12,01 \\
\hline 0,9 & 17,23 & 17,17 & 15,55 \\
\hline 1,0 & 18,16 & 18,05 & 16,57 \\
\hline
\end{tabular}

Tabela 5.3: Erro relativo referente às simulações representadas na Fig. 5.6 para Re $=300$.

Com o objetivo de investigar o desempenho dos esquemas de discretização dos termos convectivos para regime de escoamento turbulento, foram realizadas simulações para esse escoamento. Neste caso, a possibilidade de comparar os resultados numéricos com outros da literatura torna-se mais restrita, pois devido às complexidades inerentes aos modelos de turbulência, a literatura é escassa de resultados numéricos. Porém, neste trabalho, algumas simulações foram realizadas e são apresentadas a seguir.

Em grande parte das simulações feitas para o escoamento em regime turbulento, a convergência teve comportamento oscilatório quando se utilizaram os esquemas WACEB e CUBISTA, principalmente quando a malha computacional é refinada, o que não ocorreu com o esquema QUICKEST adaptativo.

De acordo com o método númerico apresentado na seção 4.2, a simulação numérica 
será concluída quando as condições (4.2.9) e (4.2.10)

$$
\begin{gathered}
R_{v}=\frac{1}{\Delta t}\left(\sum_{i=1} \sum_{j=1}\left|u_{i, j}^{n+1}-u_{i, j}^{n}\right|+\left|v_{i, j}^{n+1}-v_{i, j}^{n}\right|\right) \leq \varepsilon_{v}, \\
R_{c}=\frac{1}{\Delta t}\left(\sum_{i=1} \sum_{j=1}\left|c_{i, j}^{n+1}-c_{i, j}^{n}\right|\right) \leq \varepsilon_{c},
\end{gathered}
$$

forem satisfeitas. No caso dos esquemas WACEB e CUBISTA, observou-se nas simulações que os valores do termo $R_{v}$, calculados em cada passo de tempo, não convergiram para o valor $\varepsilon_{v}$, como pode ser observado na Fig. 5.7, a qual representa o gráfico do critério de parada para a velocidade, $R_{v}$, em função do número de iterações.

Os dados das simulações representadas na Fig. 5.7] são os seguintes:

- diâmetro: $d=0,006 m$;

- número de células computacionais: $100 \times 30$;

- número de Reynolds: $R e=10000$;

- pressão inicial: $P_{0}=2 \times 10^{5} \mathrm{~Pa}$;

- viscosidade dinâmica: $\mu=0,0013 \mathrm{Pas}^{-1}$

- massa específica: $\rho=1000 \mathrm{kgm}^{-3}$

- coeficiente de difusão: $D=8.3 \times 10^{-9} \mathrm{~m}^{2} \mathrm{~s}^{-1}$;

- resistência da membrana: $R_{m}=0,293 \times 10^{10} \mathrm{kgm}^{-2} \mathrm{~s}^{-1}$;

- resistência devido à camada de concentração: $R_{f}=1,247 \times 10^{10} \mathrm{kgm}^{-2} \mathrm{~s}^{-1}$;

- constante de proporcionalidade: $\phi=0,116 \times 10^{5} \mathrm{sm}^{-1}$;

- índice das leis de potências: $n=1,0$;

- parâmetro de convergência: $\varepsilon_{v}=10^{-8}$. 

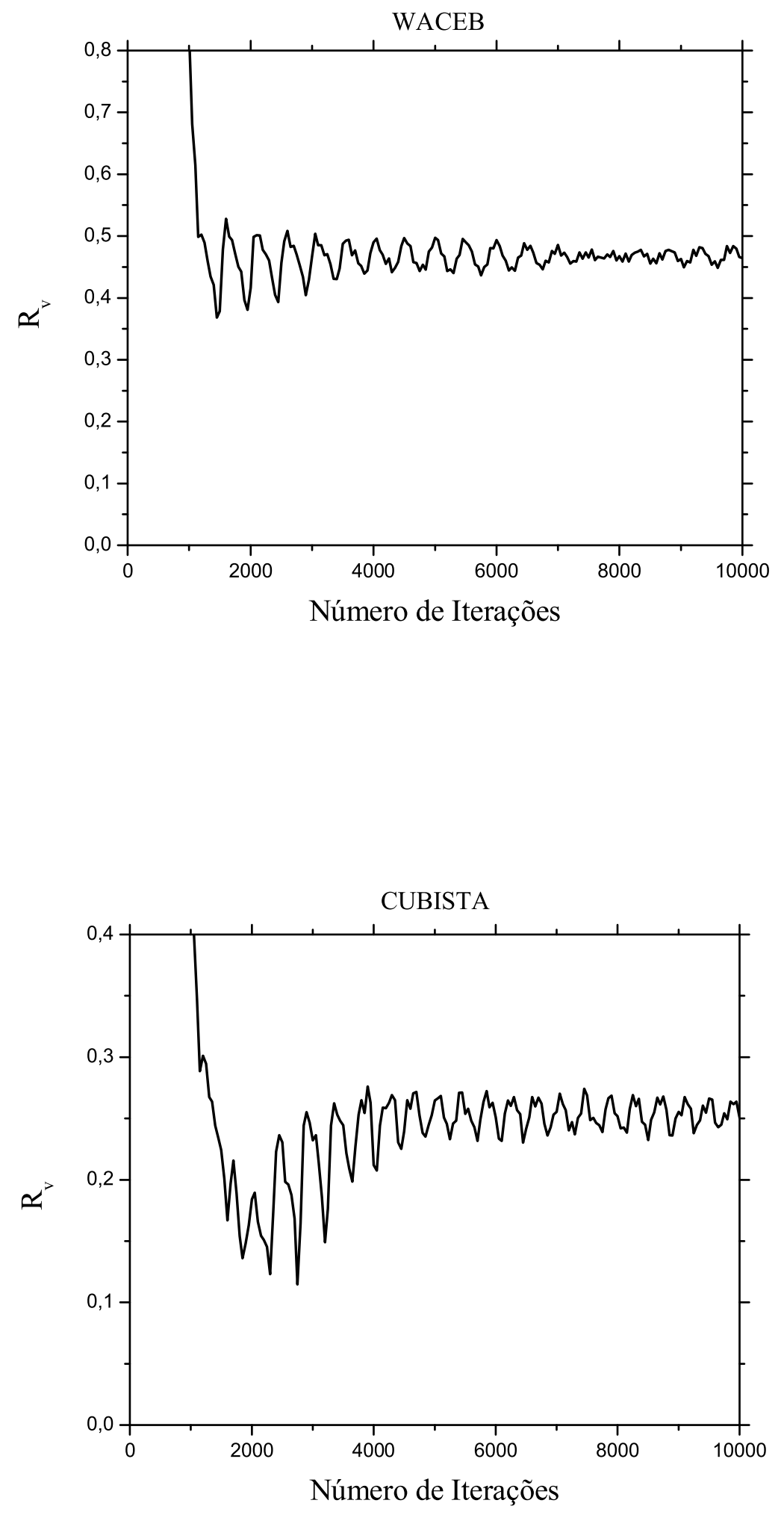


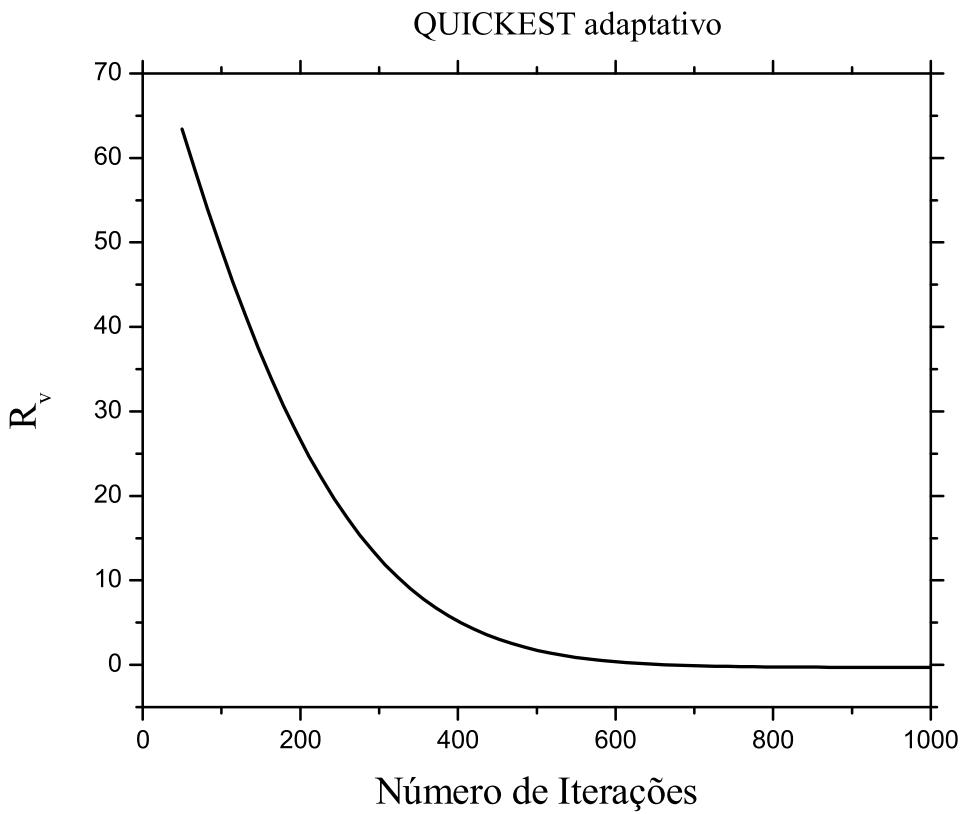

Figura 5.7: Critério de parada em função do número de iterações.

Pode-se notar na Fig. 5.7 que para o esquema QUICKEST adaptativo a convergência de $R_{v}$ para $\varepsilon_{v}$ se dá em 1000 iterações, enquanto que para os outros dois esquemas não há convergência, o que mostra que nesse caso, o esquema QUICKEST adaptativo mostrou-se mais eficaz.

Foram poucos os casos, para o regime de escoamento turbulento, em que se conseguiu convergência para os três esquemas analisados neste trabalho. As próximas figuras mostram esses resultados. Os dados das simulações representadas na Fig. 5.8 são os seguintes:

- diâmetro: $d=0,0004 m$;

- número de células computacionais: $50 \times 20$;

- número de Reynolds: $R e=10000$;

- pressão inicial: $P_{0}=2 \times 10^{5} \mathrm{~Pa}$;

- viscosidade dinâmica: $\mu=0,0013 \mathrm{Pas}^{-1}$; 
- viscosidade turbulenta: $\mu_{t}=0,0013 \mathrm{kgm}^{-1} \mathrm{~s}^{-1}$;

- massa específica: $\rho=1000 \mathrm{kgm}^{-3}$;

- coeficiente de difusão: $D=8.3 \times 10^{-8} \mathrm{~m}^{2} \mathrm{~s}^{-1}$;

- resistência da membrana: $R_{m}=0,293 \times 10^{9} \mathrm{kgm}^{-2} \mathrm{~s}^{-1}$;

- resistência devido à camada de concentração: $R_{f}=1,247 \times 10^{9} \mathrm{kgm}^{-2} \mathrm{~s}^{-1}$;

- constante de proporcionalidade: $\phi=0,116 \times 10^{5} \mathrm{sm}^{-1}$.

Na Fig. 5.8, é apresentada uma comparação entre os esquemas de discretização dos termos convectivos para a concentração próxima à superfície permeável em função da coordenada axial $z / R$, para o regime turbulento. Mais uma vez, pode-se notar que as simulações com os esquemas produziram resultados numéricos próximos, porém, o que se observou foram diferenças nos números de iterações, conforme visto na Tab. 5.4 .

\begin{tabular}{|l||c|c|}
\hline \multicolumn{3}{|c|}{ Número de Iterações } \\
\hline \hline WACEB & $n=1$ & $n=0,6$ \\
\hline CUBISTA & 2058 & 1377 \\
\hline QUICKEST adaptativo & 538 & 4769 \\
\hline
\end{tabular}

Tabela 5.4: Número de iterações referente às simulações representadas na Fig. 5.8.

Observa-se na Tab. 5.4 que o número de iterações da simulação com o esquema QUICKEST adaptativo, para $n=1$, é em média $72 \%$ menor que os produzidos pelos outros dois esquemas, e $63 \%$ para $n=0,6$. Esse mesmo fenômeno foi observado nos resultados da Fig. 5.9, a qual representa uma comparação dos esquemas com os dados de HANEDA (2006) para a velocidade de permeação média da água ${ }^{2}$ como função da pressão transmembrana inicial, em que se têm também resultados numéricos bastantes próximos, porém com uma diferença grandes entre o número de iterações, como observa-se na Tab.

\footnotetext{
${ }^{2}$ Como o fluido é a água, então a velocidade de permeação é calculada pela equação (5.1.1).
} 

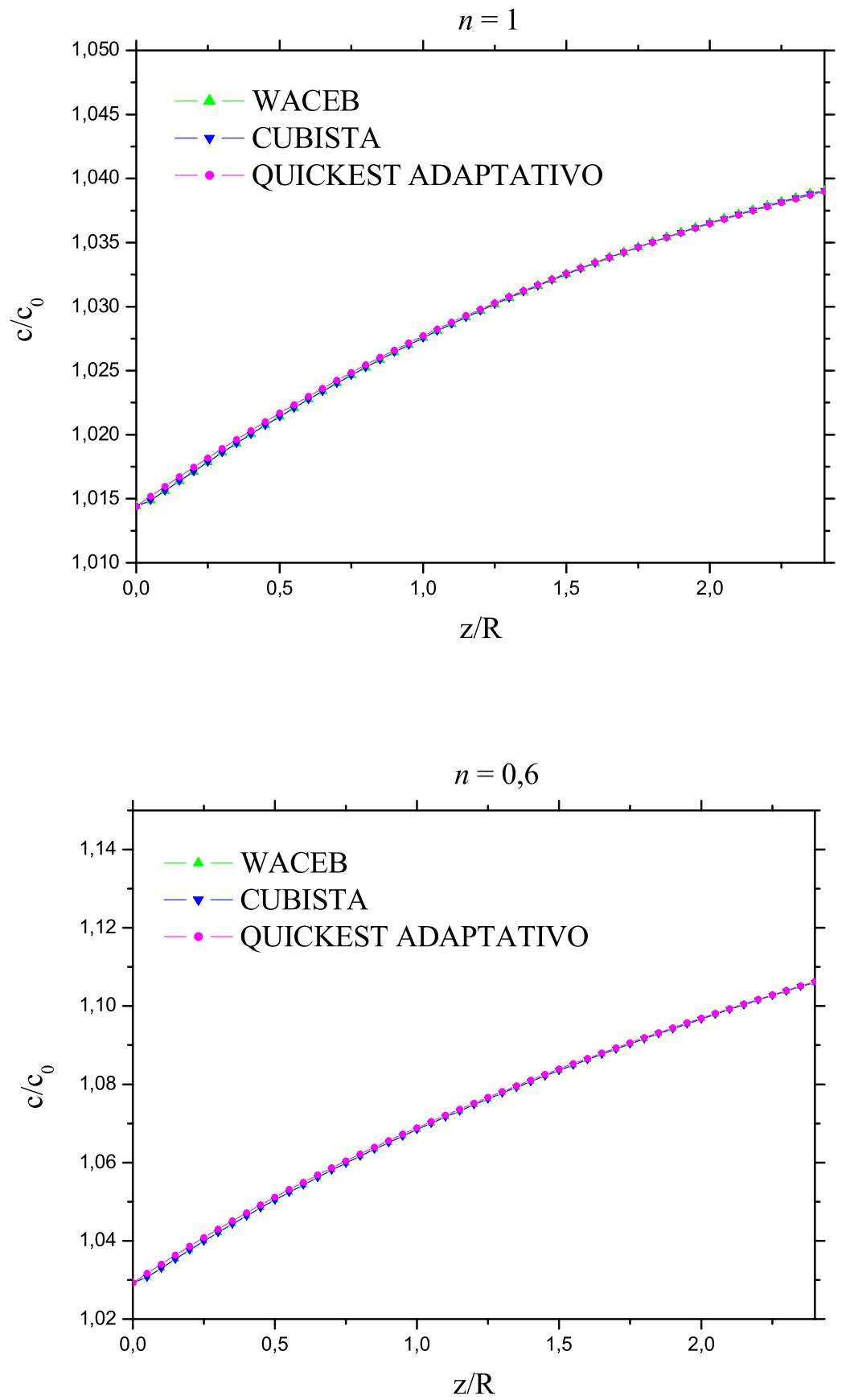

Figura 5.8: Concentração próxima à superfície da membrana em função da coordenada axial $z / R$.

5.5 .

Os dados para as simulações representadas na Fig. 5.9 são os seguintes: 
- diâmetro: $d=0,006 m$;

- número de células computacionais: $50 \times 20$;

- número de Reynolds: $R e=22690$;

- viscosidade dinâmica: $\mu=0,001 \mathrm{Pas}^{-1}$;

- massa específica: $\rho=1000 \mathrm{kgm}^{-3}$;

- resistência da membrana: $R_{m}=0,166 \times 10^{11} \mathrm{kgm}^{-2} \mathrm{~s}^{-1}$.

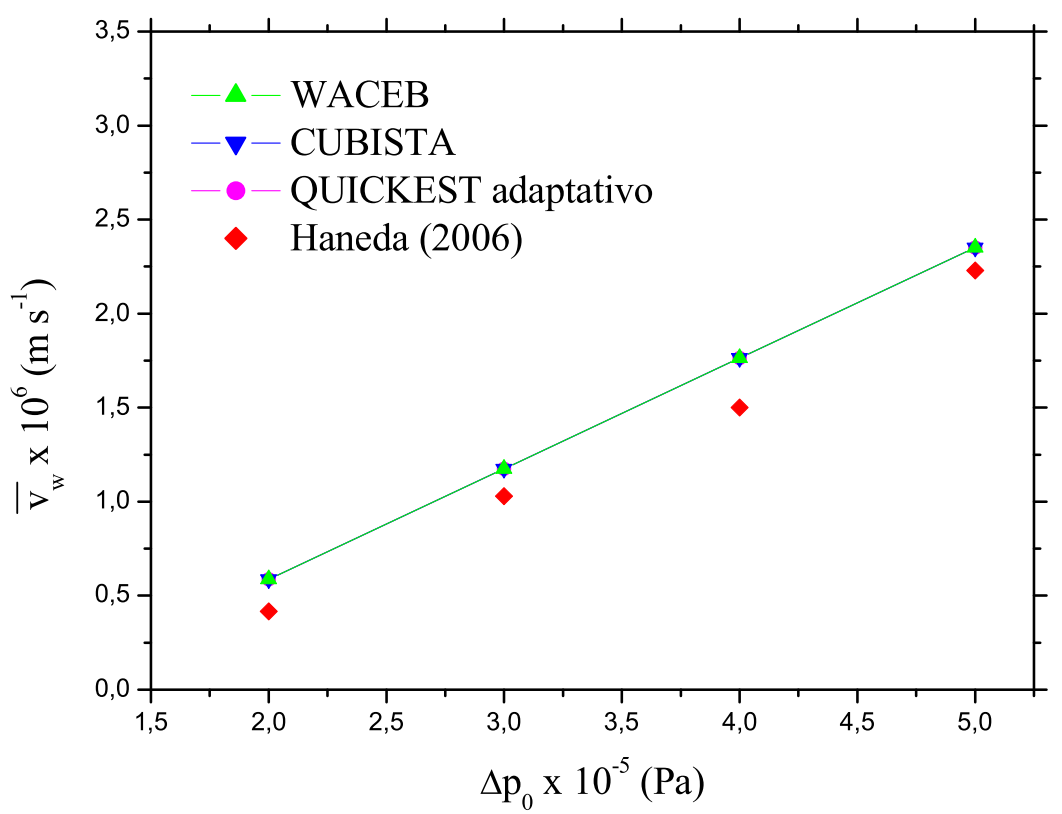

Figura 5.9: Velocidade de permeação média em função da pressão transmembrana inicial.

\begin{tabular}{|l||c|}
\hline \multicolumn{2}{|c|}{ Número de Iterações } \\
\hline \hline WACEB & 1749 \\
\hline CUBISTA & 1656 \\
\hline QUICKEST adaptativo & 389 \\
\hline
\end{tabular}

Tabela 5.5: Número de iterações referente às simulações representadas na Fig. 5.9. 
Dos resultados representados nas Figs. 5.5 e 5.6, pode-se concluir que os esquemas convectivos influenciaram nos resultados numéricos com respeito ao mecanismo de transferência na superfície permeável. Observa-se que o esquema QUICKEST adaptativo pode ser considerado como o mais apropriado para o estudo numérico, em regime laminar deste problema.

No caso do escoamento em regime turbulento, analisado a partir das Figs. 5.7, 5.8 e 5.9, tem-se que o esquema QUICKEST adaptativo apresentou uma convergência numérica com poucas iterações. Dessa forma, tanto para o regime laminar quanto para o turbulento, o esquema QUICKEST adaptativo, com um melhor desempenho, será utilizado nas simulações das seções seguintes.

\subsection{Resultados com o esquema QUICKEST adapta-}

\section{tivo}

Na seção anterior, analisaram-se alguns esquemas convectivos e sugeriu-se o esquema QUICKEST adaptativo como base para as próximas simulações. Nesta seção, o objetivo é, utilizando-se o esquema selecionado, analisar os modelos apresentados no capítulo 3 e verificar se esses produzem resultados compatíveis com o problema físico estudado. Para isso, são apresentados resultados numéricos comparados com a literatura.

\subsubsection{Resultados para escoamento em regime laminar}

Nas Fig. 5.10 e 5.11, são apresentadas comparações dos resultados numéricos deste trabalho com resultados experimentais obtidos de Yeh et al (2003) e Yeh et al (1997) rela- 
tivos à evolução da velocidade média de permeação como função da pressão transmembrana inicial para soluções de Dextran T500 (com concentração inicial: 1wt.\%) e PVP-360 (com concentração inicial: $1 w t . \%$ e $2 w t . \%$ ), as quais são fluidos newtonianos. Observa-se que os resultados numéricos dessas simulações estão bastante próximos dos dados experimentais, com erro relativo máximo de $2 \%$ para a solução de Dextran e $5,4 \%$ e $15 \%$ para as soluções de PVP-360 com concentração inicial 1wt.\% e 2wt.\%, respectivamente. As duas membranas utilizadas nesses experimentos são do tipo fibra oca.

Os dados para as simulações com Dextan T500 representadas na Fig. 5.10 são os seguintes:

- diâmetro: $d=0,0005 m$;

- número de células computacionais: $200 \times 20$;

- número de Reynolds: $R e=76$;

- viscosidade dinâmica: $\mu=0,001344 \mathrm{Pas}^{-1}$;

- massa específica: $\rho=1000 \mathrm{kgm}^{-3}$;

- coeficiente de difusão: $D=3 \times 10^{-11} \mathrm{~m}^{2} \mathrm{~s}^{-1}$;

- resistência da membrana: $R_{m}=3,674 \times 10^{9} \mathrm{kgm}^{-2} \mathrm{~s}^{-1}$;

- resistência devido à camada de concentração: $R_{f}=1,294 \times 10^{9} \mathrm{kgm}^{-2} \mathrm{~s}^{-1}$;

- constante de proporcionalidade: $\phi=2,173 \times 10^{5} \mathrm{sm}^{-1}$.

Os dados para as simulações com PVP-360 representadas na Fig. 5.11 são os seguintes:

- diâmetro: $d=0,0005 m$;

- número de células computacionais: $200 \times 20$;

- número de Reynolds: $R e=17\left(c_{0}=1 w t . \%\right)$ e $R e=7\left(c_{0}=2 w t . \%\right)$; 
- viscosidade dinâmica: $\mu=0,002145$ Pas $^{-1}\left(c_{0}=1 w t . \%\right)$ e $\mu=0,005145$ Pas $^{-1}$ $\left(c_{0}=2 w t . \%\right)$

- massa específica: $\rho=1000 \mathrm{kgm}^{-3}$;

- coeficiente de difusão: $D=3 \times 10^{-11} \mathrm{~m}^{2} \mathrm{~s}^{-1}$;

- resistência da membrana: $R_{m}=0,501 \times 10^{9} \mathrm{kgm}^{-2} \mathrm{~s}^{-1}$;

- resistência devido à camada de concentração: $R_{f}=3,346 \times 10^{9} \mathrm{kgm}^{-2} \mathrm{~s}^{-1}\left(c_{0}=1 w t . \%\right)$

e $R_{f}=24,30 \times 10^{9} \mathrm{kgm}^{-2} \mathrm{~s}^{-1}\left(c_{0}=2 w t . \%\right)$;

- constante de proporcionalidade: $\phi=3,164 \times 10^{5} \mathrm{sm}^{-1}\left(c_{0}=1 w t . \%\right) \mathrm{e}$ $\phi=3,503 \times 10^{5} \mathrm{sm}^{-1}\left(c_{0}=2 w t . \%\right)$.

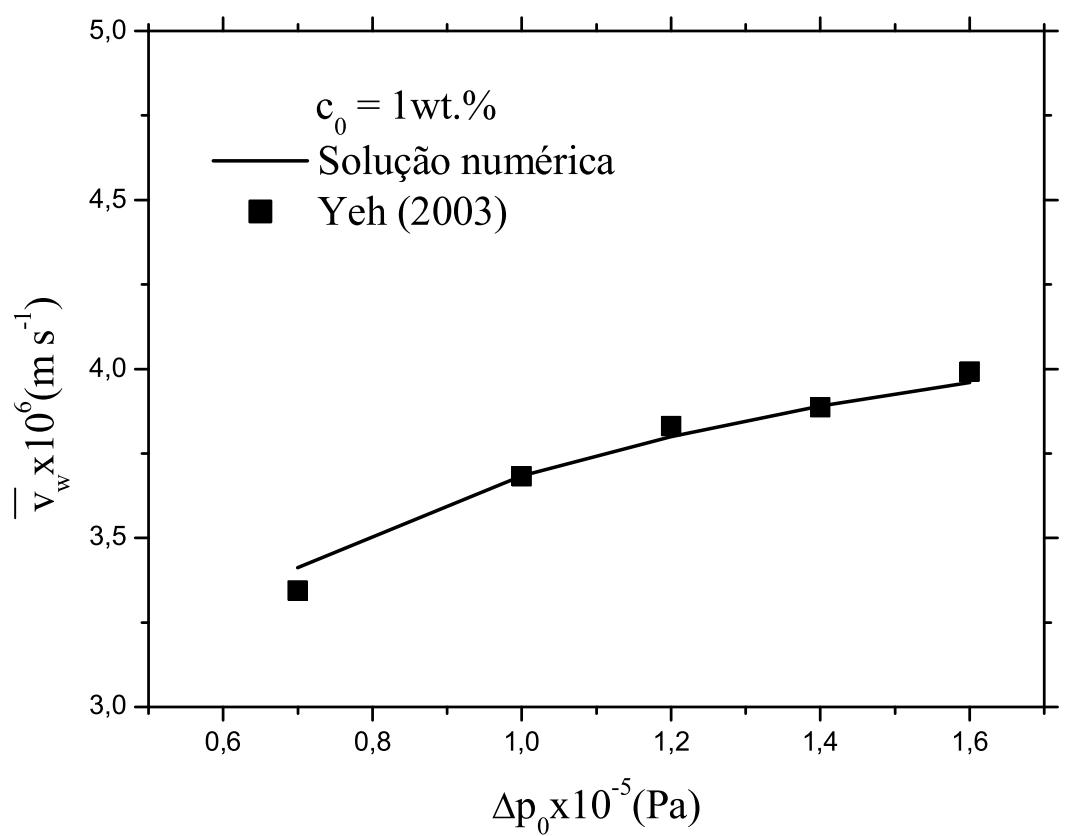

Figura 5.10: Velocidade de permeação média de uma solução de Dextran T500 em função da pressão transmembrana inicial. 

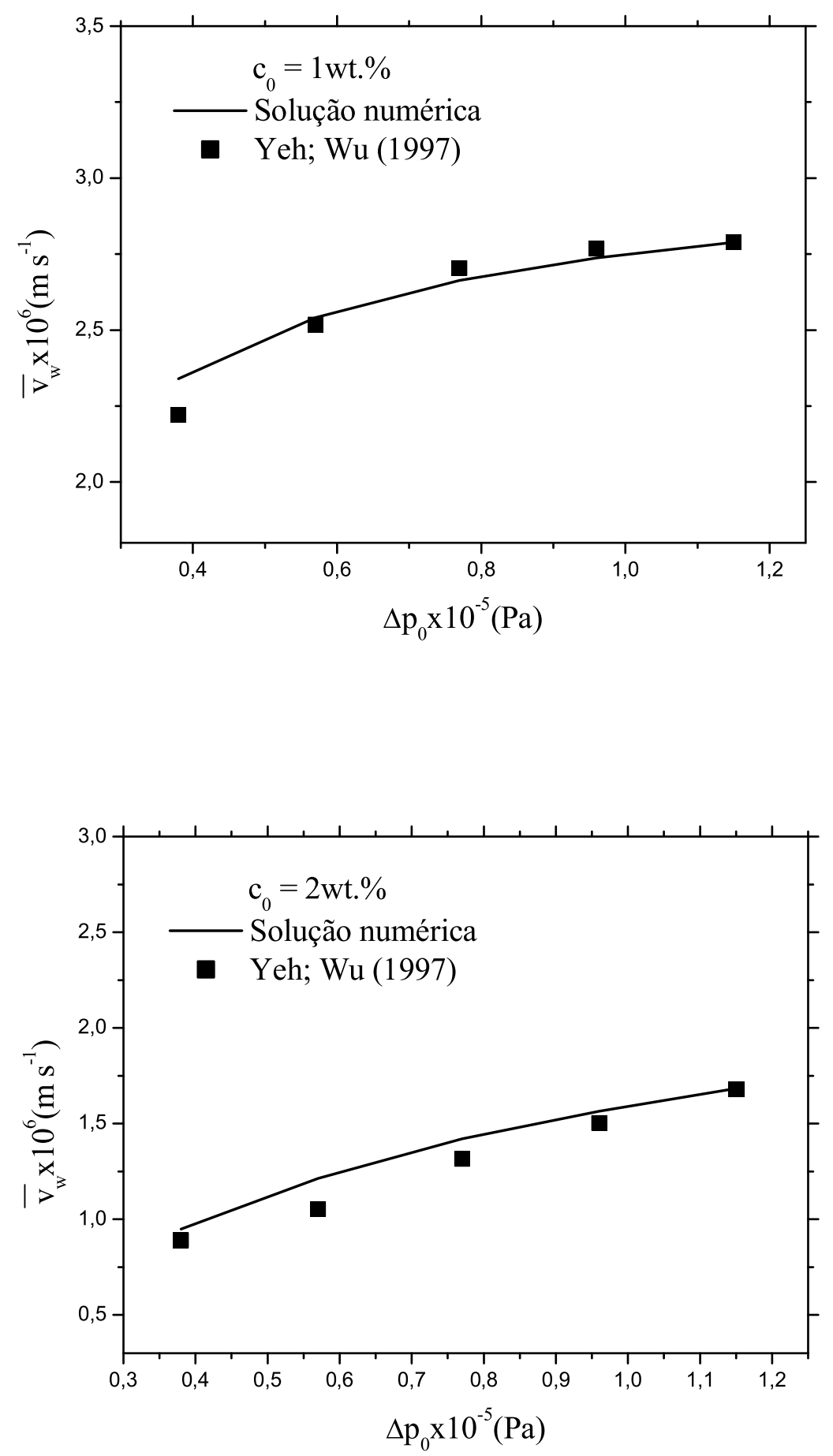

Figura 5.11: Velocidade de permeação média de uma solução de PVP-360 em função da pressão transmembrana inicial.

Os dados para as simulações representadas nas Figs. 5.12 à $[5.16$ são:

- diâmetro: $d=0,004 m$; 
- número de células computacionais: $200 \times 20$;

- número de Reynolds: $R e=100$;

- coeficiente de consistência: $m=0,000157$ Pas $^{n}$;

- massa específica: $\rho=1000 \mathrm{kgm}^{-3}$;

- coeficiente de difusão: $D=3 \times 10^{-11} \mathrm{~m}^{2} \mathrm{~s}^{-1}$;

- resistência da membrana: $R_{m}=5,88 \times 10^{9} \mathrm{kgm}^{-2} \mathrm{~s}^{-1}$;

- resistência devido à camada de concentração: $R_{f}=1,247 \times 10^{9} \mathrm{kgm}^{-2} \mathrm{~s}^{-1}$;

- constante de proporcionalidade: $\phi=0,116 \times 10^{5} \mathrm{sm}^{-1}$;

- índice de comportamento: $n=0,88$.

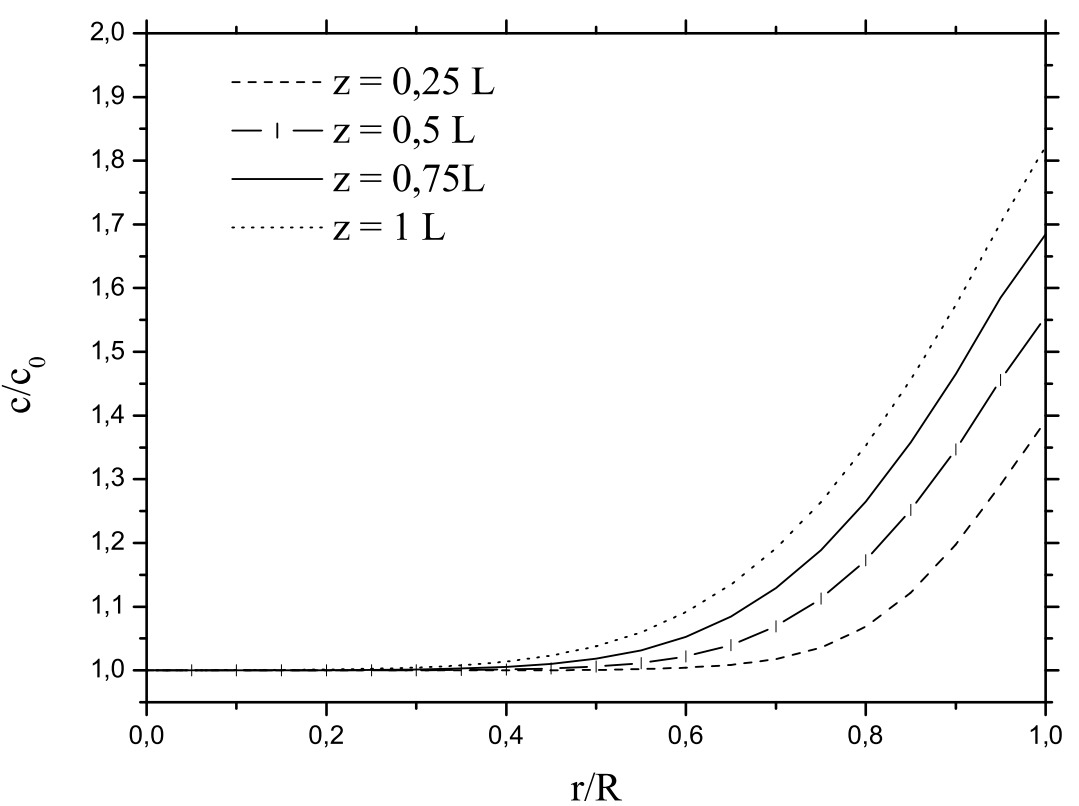

Figura 5.12: Concentração local em função da coordenada radial para diferentes localizações axiais.

Na Fig. 5.12, é mostrado o campo de concentração de um fluido não-newtoniano em função da coordenada radial, $r / R$, para várias posições axiais do tubo. Observa-se que 
a concentração próxima à parede porosa do tubo aumenta com a distância axial do tubo, isso implica que o fluxo de permeado não será constante ao longo do eixo $z / R$, mas deve decrescer com a distância axial, desde que o aumento do número de partículas de soluto próximas a parede do tubo (polarização) aumente a resistência hidráulica contra o fluxo de permeado.

Resultados da concentração próxima à superfície permeável em função da coordenada axial, $z / R$, para diferentes pressões transmembrana são apresentados na Fig. 5.13. Observa-se que a concentração próxima à parede aumenta com a pressão transmembrana inicial, isso ocorre porque quando a pressão transmembrana aumenta, o fluxo de permeado aumenta e conseqüentemente mais partículas são esperadas ser convectivamente conduzidas para a superfície da parede porosa.

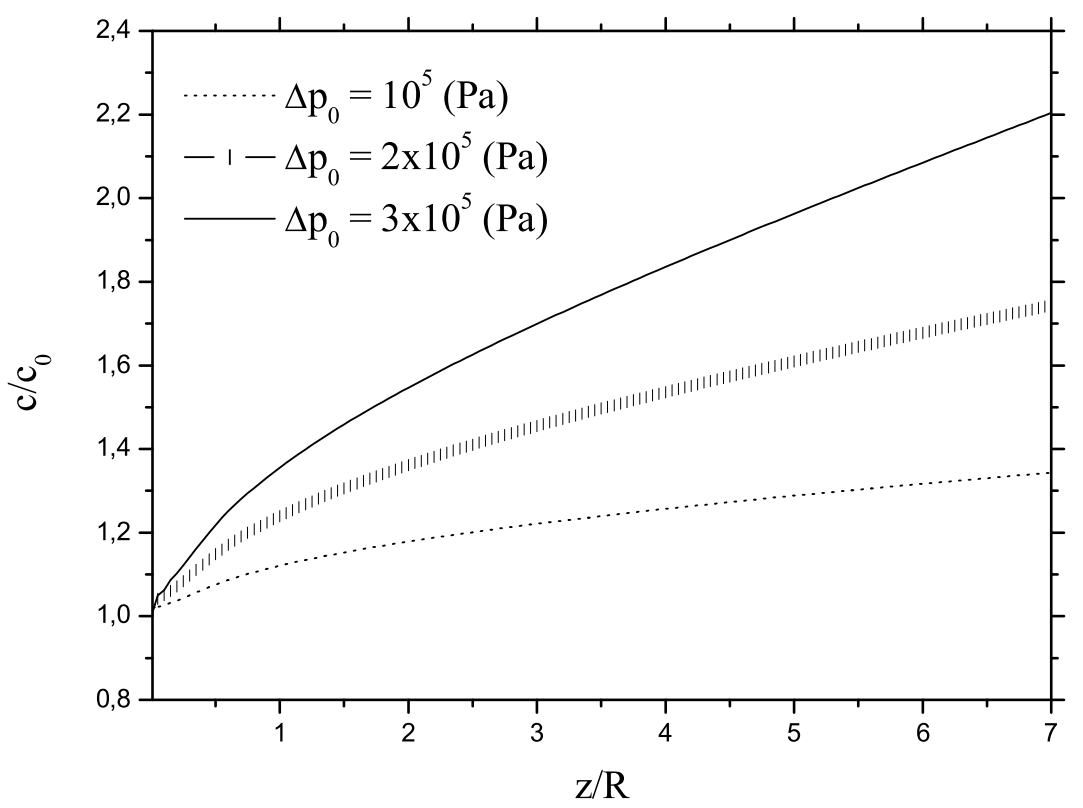

Figura 5.13: Concentração local próxima à superfície da membrana em função da coordenada radial para diferentes pressões transmembrana inicial.

Na Fig. 5.14, estão representados os perfis de concentração próximo à superfície porosa do tubo em função da coordenada axial, $z / R$, para diferentes números de Reynolds. 


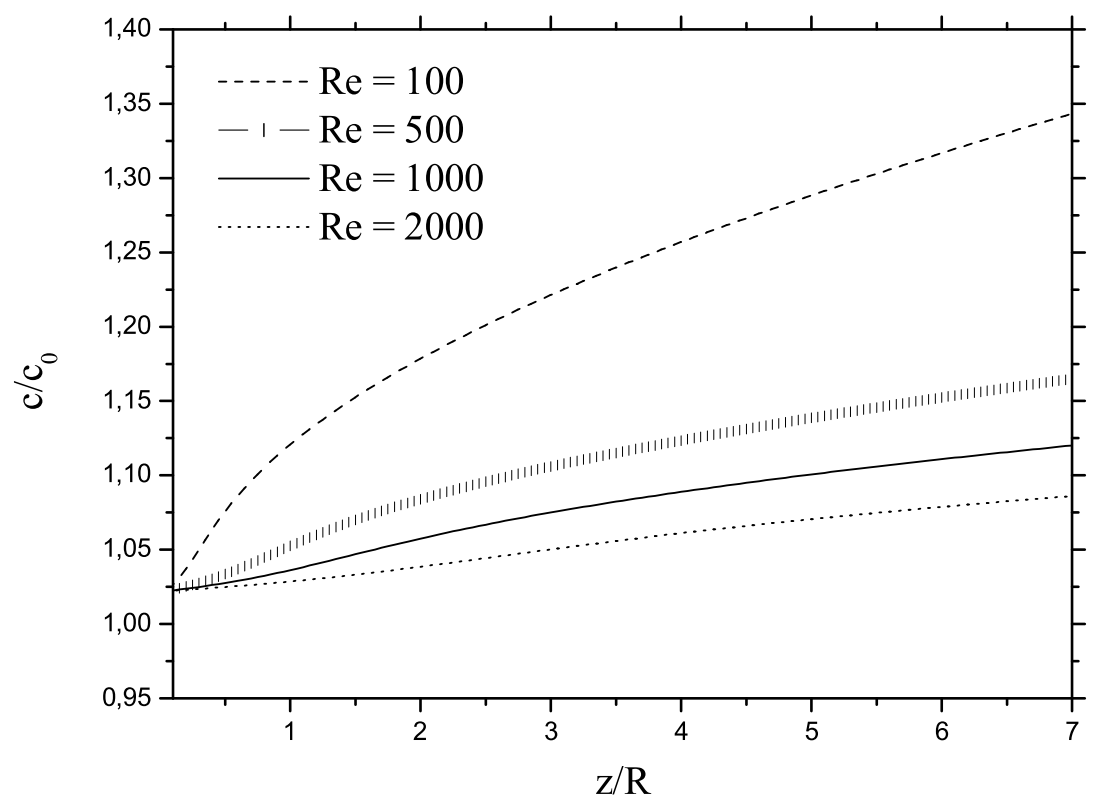

Figura 5.14: Concentração local em função da coordenada axial para diferentes números de Reynolds.

Nota-se que quando se aumenta o número de Reynolds a concentração ao longo do comprimento do tubo diminui, isso se deve ao fato de que com o aumento do números Reynolds, têm-se maiores velocidades axiais, o que diminui o acúmulo de solutos na superfície porosa. Pode-se observar essa tendência também na Fig. 5.15, que representa o perfil de concentração em função da coordenada radial, $r / R$, em $z / R=0,5 L / R$, para diferentes números de Reynolds.

Na Fig. 5.16, estão representados os gráficos do número de Sherwood local em função da coordenada axial $z / R$, para diferentes números de Reynolds. Como o número de Sherwood é um adimensional que quantifica a taxa de transferência de massa, observase que os valores do número de Sherwood local aumentam com o aumento do número de Reynolds. Essa correlação típica de problemas convectivos, indica que o aumento do Reynolds realiza uma ação cisalhante na superfície porosa capaz de reduzir a polarização. 
Assim, em geral, tem-se o aumento da vazão transmembrana, até o limite do fluxo crítico ([BACCHIN et al., 2005]).

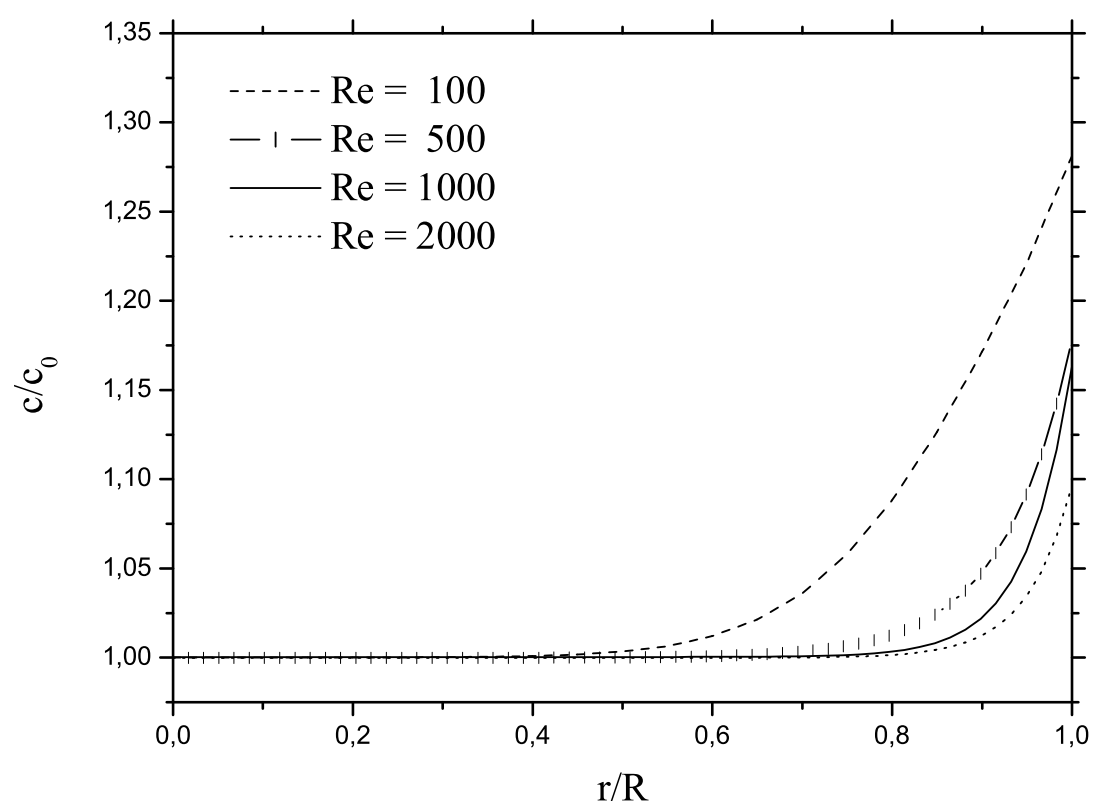

Figura 5.15: Concentração local em função da coordenada radial para diferentes números de Reynolds.

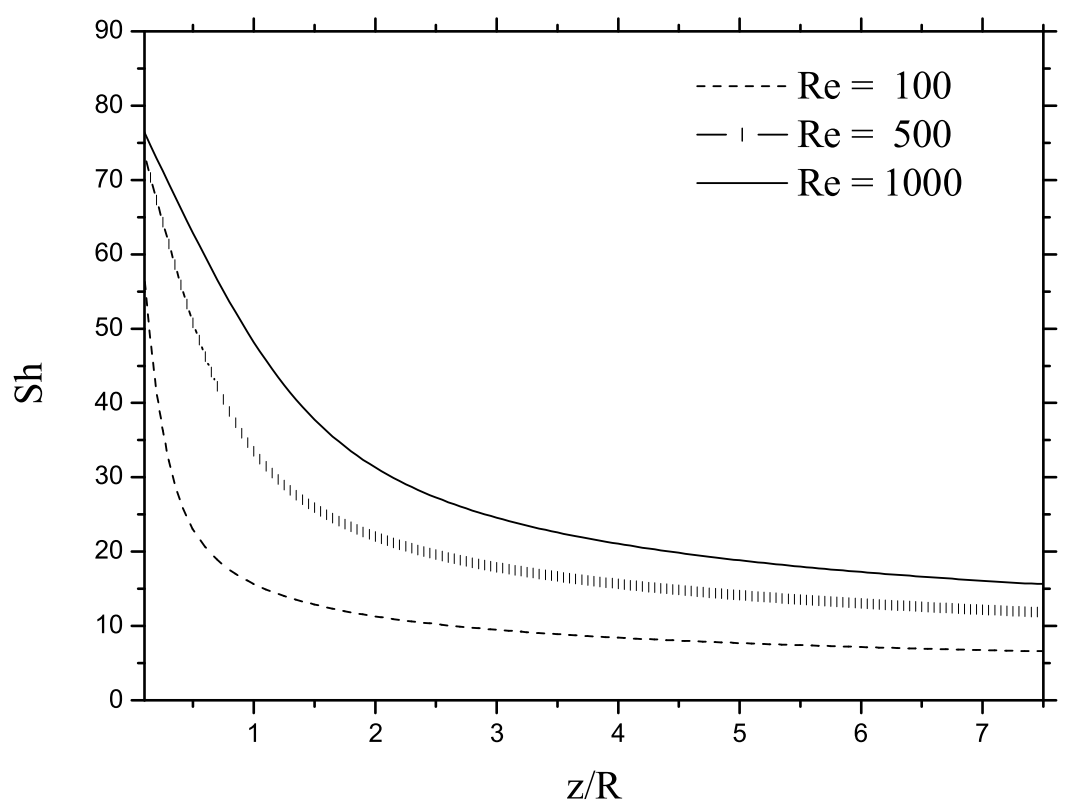

Figura 5.16: Número de Sherwood em função da coordenada axial para diferentes números de Reynolds. 
Com os resultados apresentados nas Figs. 5.10 à 5.16, observou-se que o modelo matemático para o regime de escoamento laminar produziu resultados quantitativamente e qualitativamente compatíveis com a literatura.

\subsubsection{Resultados para escoamento em regime turbulento}

Na Fig. 5.17, é apresentado o perfil de concentração próximo à superfície da membrana em função da coordenada axial, $z / R$, para diferentes pressões transmembrana. Observa-se que a concentração próxima à superfície permeável aumenta com o aumento da pressão transmembrana, isso se deve ao acréscimo da força motriz na direção normal à superfície porosa causada da pressão transmembrana. Outra observação importante a respeito dos resultados na Fig. 5.17, é o fato dos valores da concentração se aproximarem com o aumento da pressão transmembrana. Tal comportamento tem sido estudado e observado em processos de microfiltração ([BACCHIN et al., 2005]). O aumento contínuo da pressão transmembrana não induz um aumento linear no fluxo transmembrana e não deve promover um aumento correspondente à concentração na superfície. Isso pode ser observado na Fig. 5.18 que descreve o rápido crescimento do fluxo de permeado até à pressão transmembrana inicial igual a $3 \times 10^{5} \mathrm{~Pa}$ e logo após um crescimento gradual até um valor limite.

Os dados para as simulações representadas nas Fig. 5.17 e 5.18 são os seguintes:

- diâmetro: $d=0,006 m$;

- número de células computacionais: $300 \times 30$;

- número de Reynolds: $R e=1,9 \times 10^{5}$;

- coeficiente de consistência: $m=0,00058 \mathrm{Pas}^{n}$;

- massa específica: $\rho=1000 \mathrm{kgm}^{-3}$; 
- coeficiente de difusão: $D=8,3 \times 10^{-11} \mathrm{~m}^{2} \mathrm{~s}^{-1}$;

- resistência da membrana: $R_{m}=0,293 \times 10^{11} \mathrm{kgm}^{-2} \mathrm{~s}^{-1}$;

- resistência devido à camada de concentração: $R_{f}=1,247 \times 10^{11} \mathrm{kgm}^{-2} \mathrm{~s}^{-1}$;

- constante de proporcionalidade: $\phi=0,116 \times 10^{7} \mathrm{sm}^{-1}$;

- índice de comportamento: $n=0,84$.

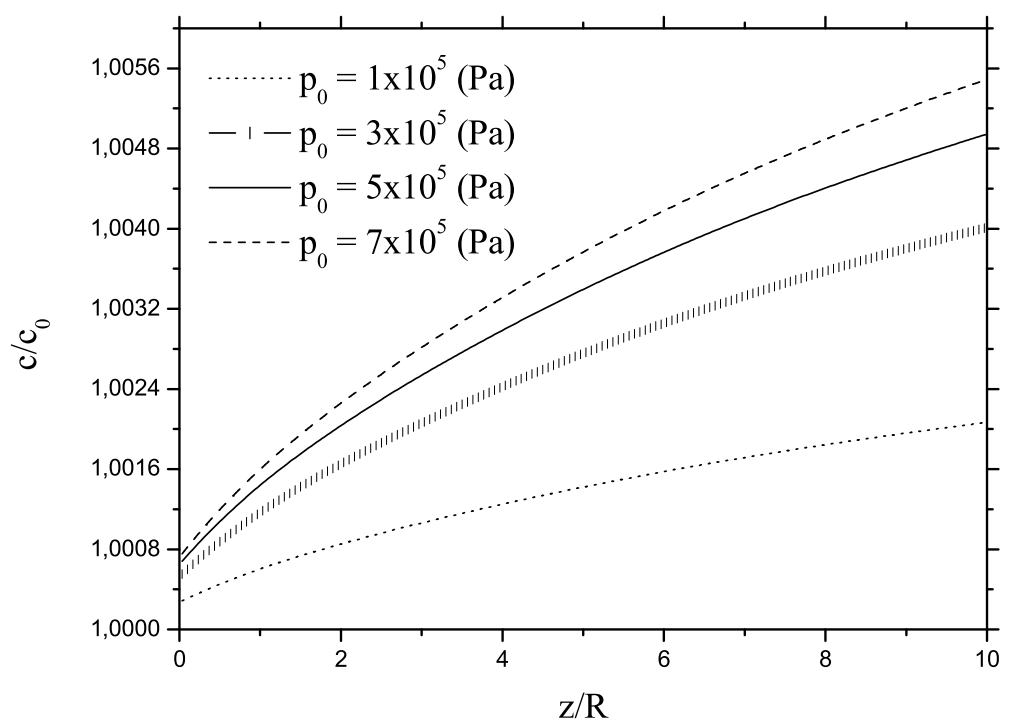

Figura 5.17: Concentração próxima à superfície da membrana em função da coordenada axial, para diferentes pressões transmembrana.

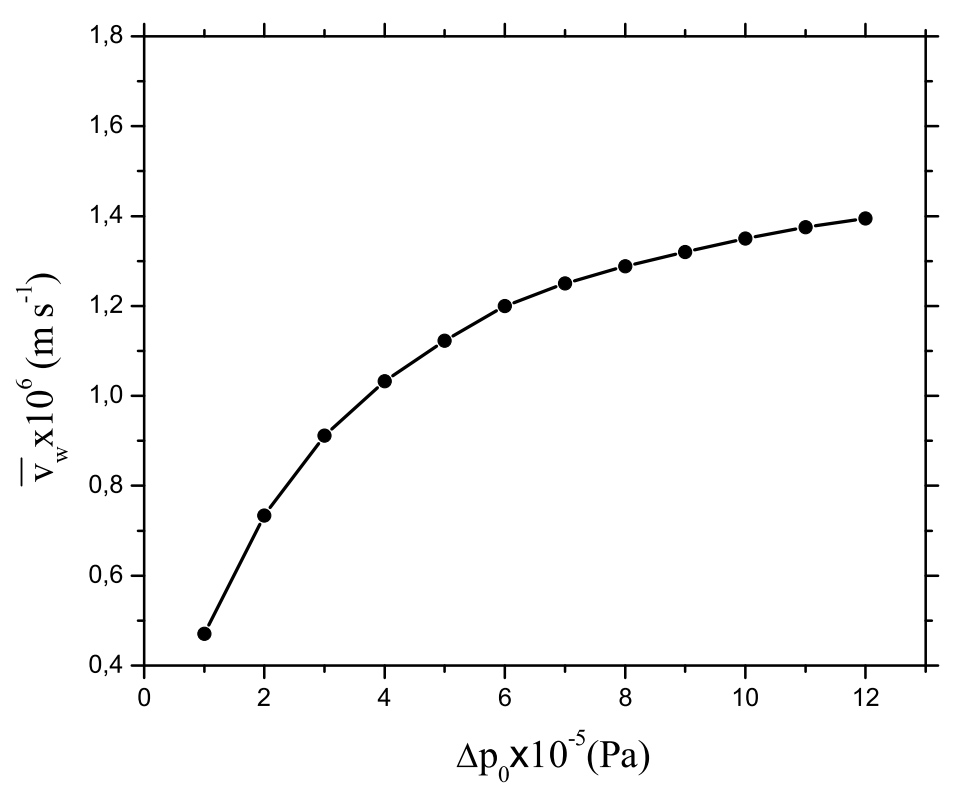

Figura 5.18: Velocidade de permeação média em função da pressão transmembrana inicial. 
É apresentada na Fig. 5.19 uma comparação dos resultados numéricos com resultados experimentais de Queiroz (2004), para a velocidade de permeação média em função do número de Reynolds, decorrentes da microfiltração (membrana de $0,4 \mu m$ ) de soluções de goma guar (1000 ppm). Observa-se que os resultados numéricos possuem uma boa concordância com os resultados da literatura com errro relativo máximo de 2,035\%. Os dados das simulações, representativo dos quatro pontos na Fig. 15.19, são os seguintes:

- diâmetro: $d=0,006 m$;

- massa específica: $\rho=1000 \mathrm{kgm}^{-3}$;

- coeficiente de difusão: $D=8,3 \times 10^{-8} \mathrm{~m}^{2} \mathrm{~s}^{-1}$;

- resistência da membrana: $R_{m}=5,88 \times 10^{8} \mathrm{kgm}^{-2} \mathrm{~s}^{-1}$;

- pressão inicial: $P_{0}=3 \times 10^{5} \mathrm{~Pa}$;

(i) número de Reynolds: $R e=6,9 \times 10^{4}$;

- coeficiente de consistência: $m=0,00029$ Pas $^{n}$;

- resistência devido à camada de concentração: $R_{f}=5,8 \times 10^{11} \mathrm{kgm}^{-2} \mathrm{~s}^{-1}$;

- constante de proporcionalidade: $\phi=0,0876 \times 10^{6} \mathrm{sm}^{-1}$;

- índice de comportamento: $n=0,97$;

(ii) número de Reynolds: $R e=1,1 \times 10^{5}$;

- coeficiente de consistência: $m=0,00042$ Pas $^{n}$;

- resistência devido à camada de concentração: $R_{f}=0,460326 \times 10^{11} \mathrm{kgm}^{-2} \mathrm{~s}^{-1}$;

- constante de proporcionalidade: $\phi=0,2158 \times 10^{7} \mathrm{sm}^{-1}$;

- índice de comportamento: $n=0,91$;

(iii) número de Reynolds: $R e=1,4 \times 10^{5}$;

- coeficiente de consistência: $m=0,00036$ Pas $^{n}$; 
- resistência devido à camada de concentração: $R_{f}=0,4484 \times 10^{11} \mathrm{kgm}^{-2} \mathrm{~s}^{-1}$;

- constante de proporcionalidade: $\phi=0,1764 \times 10^{7} \mathrm{sm}^{-1}$;

- índice de comportamento: $n=0,93$;

(iv) número de Reynolds: $R e=2,4 \times 10^{5}$;

- coeficiente de consistência: $m=0,00058 \mathrm{Pas}^{n}$;

- resistência devido à camada de concentração: $R_{f}=1,247 \times 10^{11} \mathrm{kgm}^{-2} \mathrm{~s}^{-1}$;

- constante de proporcionalidade: $\phi=0,116 \times 10^{7} \mathrm{sm}^{-1}$;

- índice de comportamento: $n=0,84$.

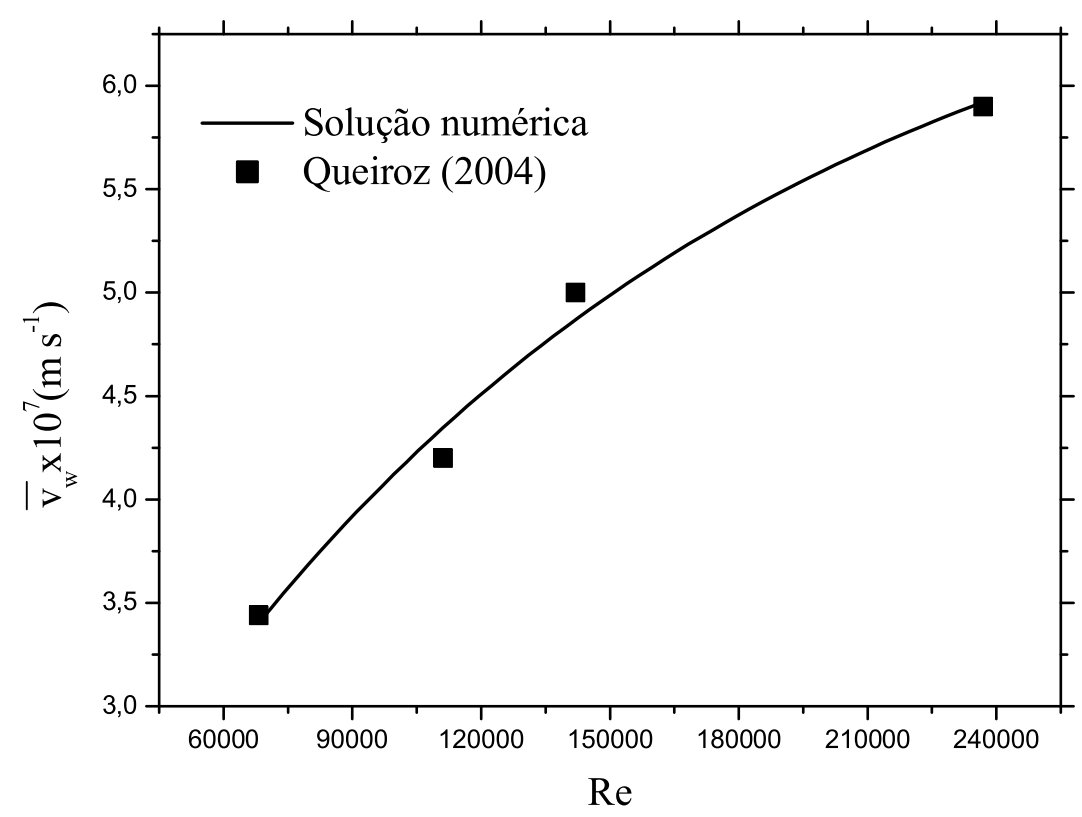

Figura 5.19: Velocidade de permeação média em função do número de Reynolds.

Na Fig. 5.20, são apresentados resultados numéricos da velocidade de permeação média em função da pressão transmembrana inicial comparados com resultados experimentais de uma solução de goma guar (2000 ppm), em uma membrana multicanal de poro 1,2 $2 \mu \mathrm{m}$. Esses dados experimentais foram gerados especialmento para este trabalho em equipamento ${ }^{3}$ do laboratorio do Núcleo de Engenharia Térmica e Fluidos (NETeF) da

\footnotetext{
${ }^{3}$ Detalhes do equipamento estão em QUEIROZ (2004) e HANEDA (2006).
} 
USP/São Carlos. Apesar dos resultados numéricos apresentarem um erro relativo máximo de $25 \%$, ainda assim, pode-se considerar uma boa concordância entre os resultados, pois os termos $R_{f}$ e $\phi$, do modelo de resistência em série, são tomados como constantes, o que se espera causar algumas diferenças entre os valores da delocidade de permeação média, $\bar{v}_{w}$.

Os dados das simulações representadas na Fig. 5.20 são os seguintes:

- diâmetro: $d=0,006 m$;

- número de células computacionais: $300 \times 30$;

- número de Reynolds: $R e=4,2 \times 10^{3}$;

- viscosidade dinâmica: $\mu=0,001 \mathrm{Pas}^{-1}$;

- massa específica: $\rho=1000 \mathrm{kgm}^{-3}$;

- coeficiente de difusão: $D=8,3 \times 10^{-8} \mathrm{~m}^{2} \mathrm{~s}^{-1}$;

- resistência da membrana: $R_{m}=9,83 \times 10^{9} \mathrm{kgm}^{-2} \mathrm{~s}^{-1}$;

- resistência devido à camada de concentração: $R_{f}=8,8 \times 10^{10} \mathrm{kgm}^{-2} \mathrm{~s}^{-1}$;

- constante de proporcionalidade: $\phi=8.096 \times 10^{4} \mathrm{sm}^{-1}$;

- índice de comportamento: $n=1,0$.

Resultados numéricos deste trabalho para o regime de escoamento turbulento também foram comparados com correlações e expressões analíticas da literatura para o número de Sherwood médio e local. Na Fig. 5.21, é apresentada uma comparação do número de Sherwood médio em função do número de Reynolds com duas correlações de Gekas e Hallström (1987 apud [CLAPP, 1963], |YYO and HARTNETT, 1974]). Ambas as correlações foram desenvolvidas para escoamento de fluidos não-newtonianos em tubos impermeáveis. Como são correlações derivadas de resultados experimentais ou baseadas em outros modelos, observa-se uma diferença entre seus resultados. Os resultados numéricos melhor se aproximam do resultados da correlação de Gekas e Hallström (1987 apud CLAPP, 1963) 


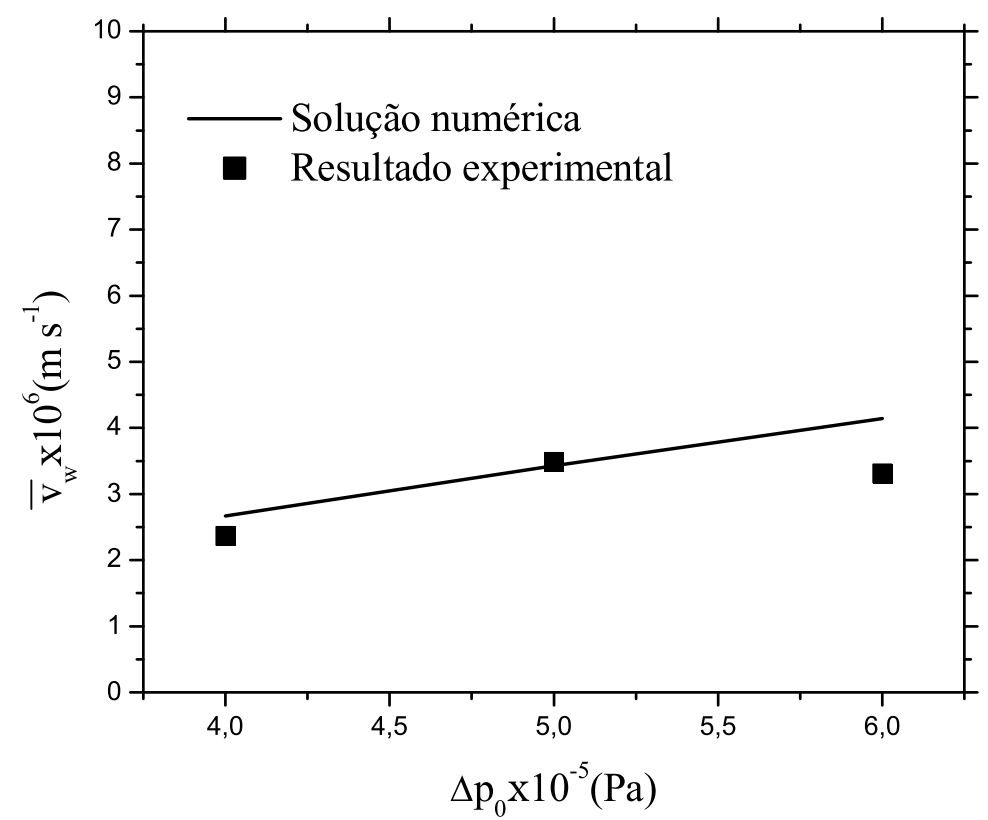

Figura 5.20: Velocidade média de permeação em função da pressão transmembrana inicial.

com erro relativo máximo de 11,2\%, enquanto que para a correlação de Gekas e Hallström (1987 apud [YOO and HARTNETT, 1974]) o erro relativo máximo é de 34,5\%.

Os dados das simulações representadas na Fig. 5.21 são os seguintes:

- diâmetro: $d=0,006 m$;

- pressão inicial: $P_{0}=2 \times 10^{5} \mathrm{~Pa}$;

- coeficiente de consistência: $m=0,0002 \mathrm{Pas}^{n}$;

- massa específica: $\rho=1000 \mathrm{kgm}^{-3}$;

- coeficiente de difusão: $D=8,3 \times 10^{-8} \mathrm{~m}^{2} \mathrm{~s}^{-1}$;

- resistência da membrana: $R_{m}=0,25 \times 10^{10} \mathrm{kgm}^{-2} \mathrm{~s}^{-1}$;

- resistência devido à camada de concentração: $R_{f}=1,5 \times 10^{10} \mathrm{kgm}^{-2} \mathrm{~s}^{-1}$;

- constante de proporcionalidade: $\phi=2 \times 10^{5} \mathrm{sm}^{-1}$;

- índice de comportamento: $n=0.9$. 


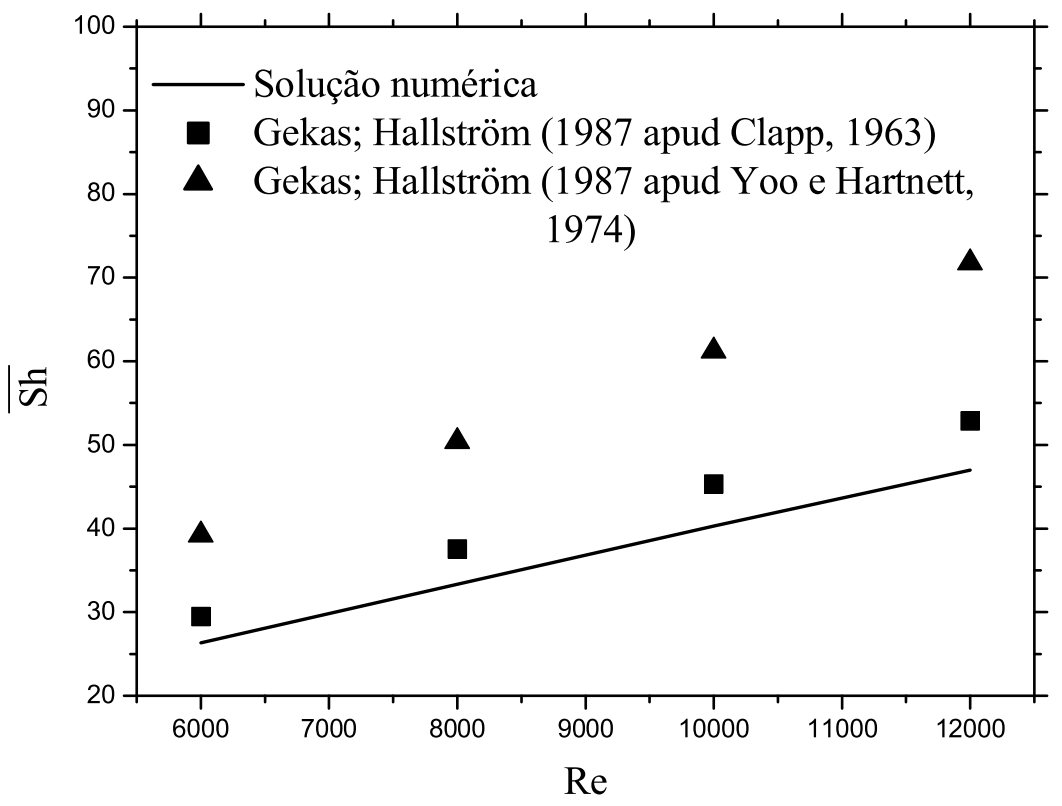

Figura 5.21: Número de Sherwood médio em função do número de Reynolds.

Uma outra comparação é apresentada na Fig. 5.22, na qual se tem novamente o número de Sherwood médio em função do número de Reynolds. Os dados da literatura foram obtidos das correlações utilizadas na Fig. 5.21 e da expressão analítica para escoamento em tubos permeáveis desenvolvida por Parvatiyar (1998), nesse caso o erro relativo máximo foi de 22\%, enquanto que para as correlações de Gekas e Hallström (1987 apud [CLAPP, 1963], YYOO and HARTNETT, 1974]) os erros relativos máximos foram de $85,5 \%$ e $81,7 \%$, respectivamente. Essa diferença pode ser atribuida às propriedades reológicas da solução (índice de comportamento, $n$, e coeficiente de consistência, $m$ ), as quais têm influência direta na viscosidade aparente, e ao fato de que a expressão analítica é para escoamentos em tubos permeáveis enquanto que as correlações são para tubos impermeáveis.

Os dados das simulações representadas na Fig. 5.22 são os seguintes:

- diâmetro: $d=0,006 m$; 
- pressão inicial: $P_{0}=2 \times 10^{5} \mathrm{~Pa}$;

- coeficiente de consistência: $m=0,03 \mathrm{Pas}^{n}$;

- massa específica: $\rho=1000 \mathrm{kgm}^{-3}$;

- coeficiente de difusão: $D=3 \times 10^{-8} \mathrm{~m}^{2} \mathrm{~s}^{-1}$;

- resistência da membrana: $R_{m}=0,25 \times 10^{10} \mathrm{kgm}^{-2} \mathrm{~s}^{-1}$;

- resistência devido à camada de concentração: $R_{f}=1,5 \times 10^{10} \mathrm{kgm}^{-2} \mathrm{~s}^{-1}$;

- constante de proporcionalidade: $\phi=2 \times 10^{5} \mathrm{sm}^{-1}$;

- índice de comportamento: $n=0.88$.

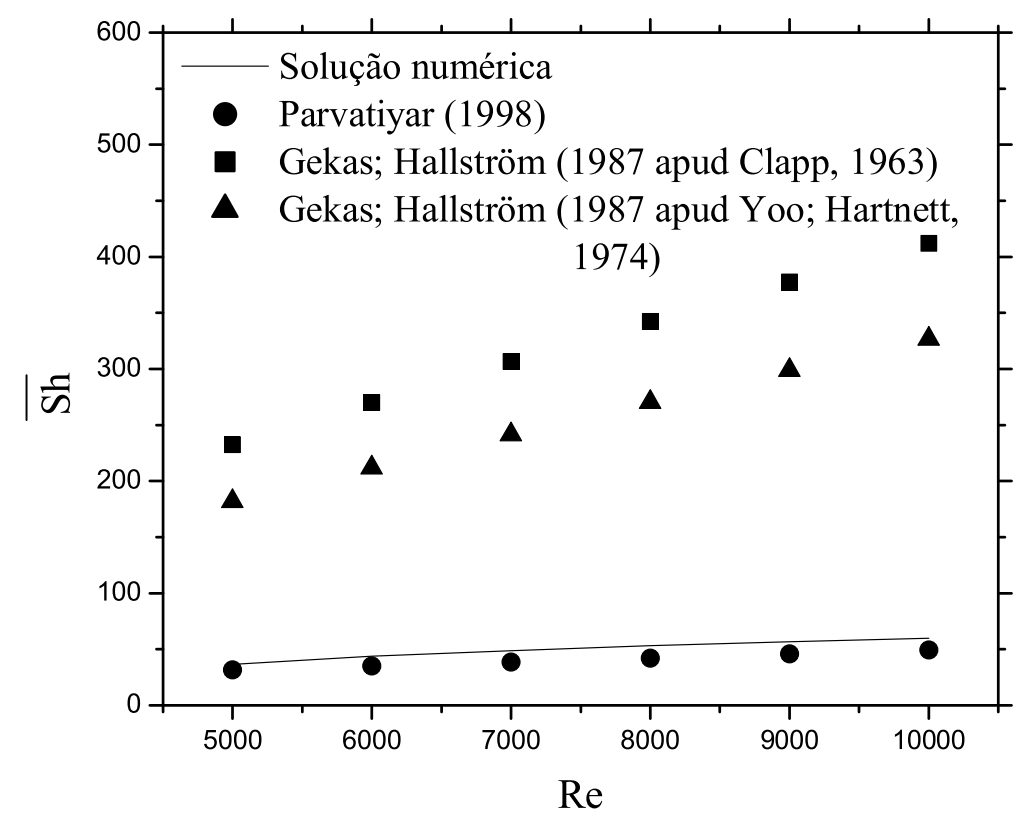

Figura 5.22: Número de Sherwood médio em função do número de Reynolds.

Uma última comparação é mostrada na Fig. 5.23 em que se tem o número de Sherwood em função da coordenada axial, $z / R$, para índices de comportamento, $n$, iguais a 0,7, 0,8 e 0,9. Os dados das simulações representadas na Fig. 5.23 são os seguintes:

- diâmetro: $d=0,006 m$; 
- número de células computacionais: $300 \times 30$;

- número de Reynolds: $R e=6 \times 10^{3}$;

- pressão inicial: $P_{0}=3 \times 10^{5} P a$;

- coeficiente de consistência: $m=0,0003$ Pas $^{n}$;

- massa específica: $\rho=1000 \mathrm{kgm}^{-3}$;

- coeficiente de difusão: $D=8,3 \times 10^{-8} \mathrm{~m}^{2} \mathrm{~s}^{-1}$;

- resistência da membrana: $R_{m}=0,25 \times 10^{10} \mathrm{kgm}^{-2} \mathrm{~s}^{-1}$;

- resistência devido à camada de concentração: $R_{f}=1,5 \times 10^{10} \mathrm{kgm}^{-2} \mathrm{~s}^{-1}$;

- constante de proporcionalidade: $\phi=2 \times 10^{5} \mathrm{sm}^{-1}$;

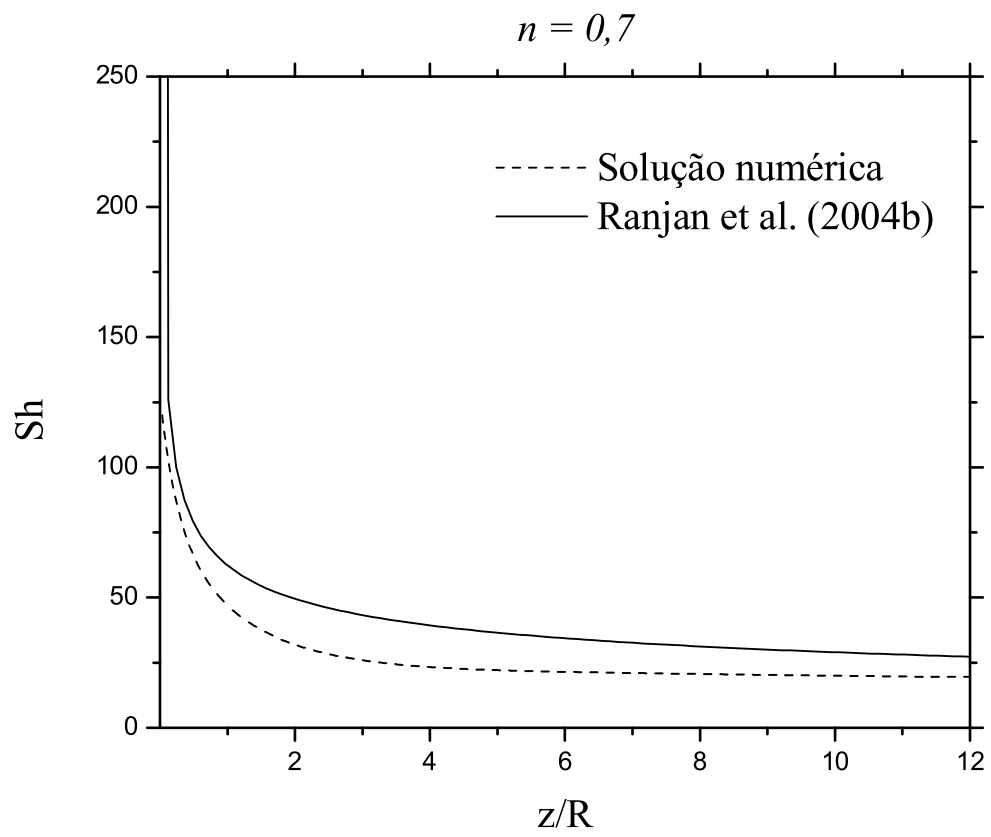



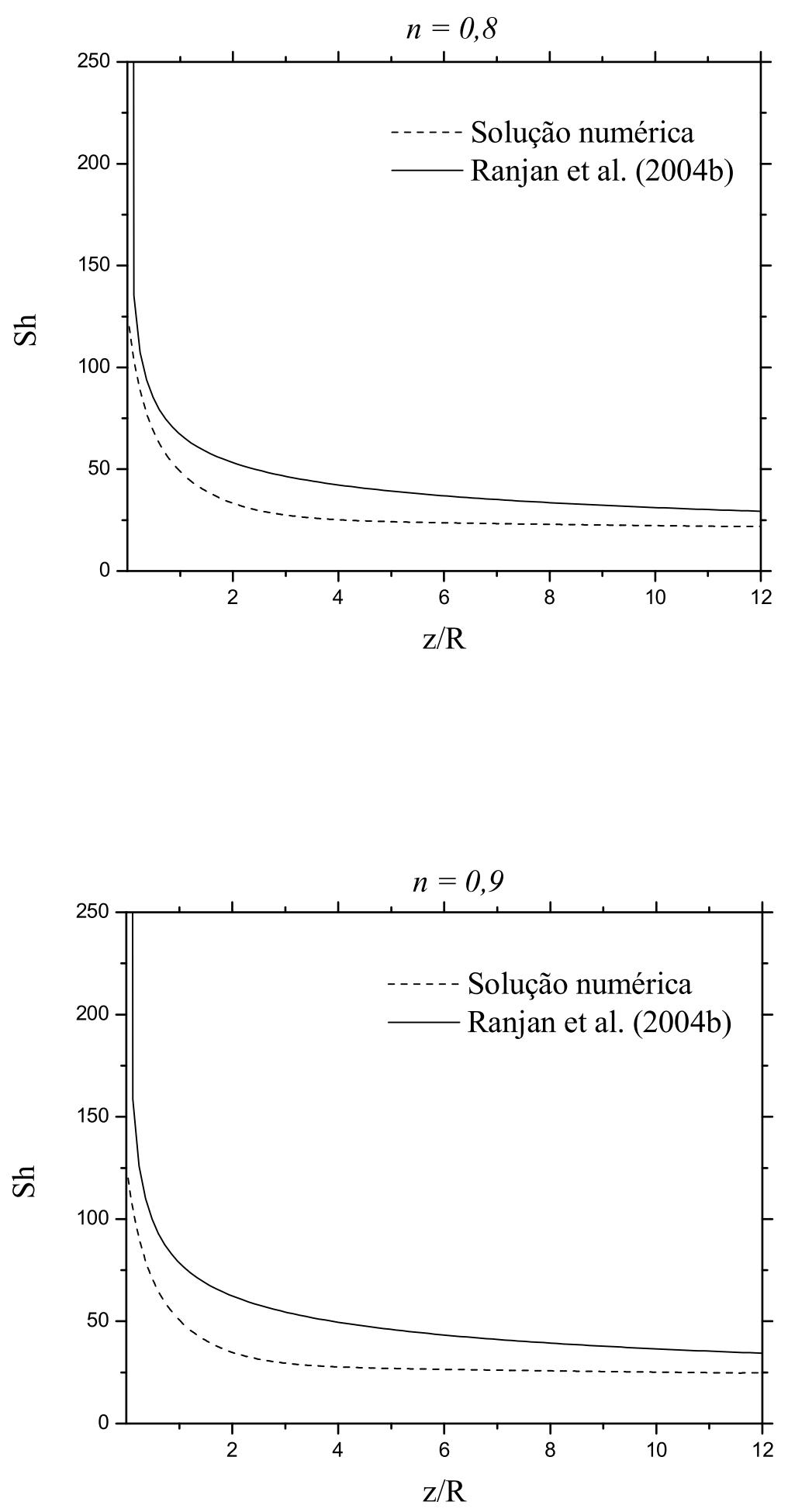

Figura 5.23: Comparação do número de Sherwood local em função da coordenada axial. 
Observa-se que os resultados numéricos possuem boa concordância assintótica com os resultados obtidos da formulação analítica de Ranjan et al. (2004b) a partir de $z / R=12$. 


\section{Conclusões e Sugestões de Trabalhos Futuros}

Neste trabalho, foi apresentada uma modelagem matemática capaz de simular escoamentos em tubos permeáveis para regimes laminar e turbulento de fluidos newtonianos e não-newtonianos aplicada ao processo de filtração tangencial. As equações de conservação, com condições de contorno apropriadas para o problema associado à filtração tangencial, foram discretizadas por diferenças finitas e o campo de pressão e velocidades calculado pelo método Sola. Devido ao problema ser governado com forte influência convectiva, a discretização dos termos convectivos nas equações de conservação foi investigada para três esquemas convectivos: WACEB, CUBISTA e QUICKEST adaptativo. A partir das simulações realizadas para análise dessa modelagem, e comparações com outras investigações da literatura, pode-se concluir que:

(i) com as simulações realizadas para os esquemas convectivos em regime de escoamento laminar e turbulento, infere-se que o esquema QUICKEST adaptativo é o de melhor desempenho numérico na modelagem desse escoamento;

(ii) as simulações comparativas com resultados da literatura, para o regime de escoa- 
mento laminar, indicam que a modelagem representa o escoamento associado à filtração tangencial;

(iii) a modelagem para processos em regime de escoamento turbulento, perante comparações com resultados da literatura (Figs. 5.19 a 5.23), indicou tendências satisfatórias, porém, deve-se ressaltar que o simplificado modelo de comprimento de mistura de Prandtl é freqüentemente utilizado na literatura para escoamentos em tubos impermeáveis, o que difere deste trabalho, que investiga o escomento em tubos permeáveis. Dessa forma, acredita-se que o modelo turbulento pode merecer aprimoramentos para melhor representar os mecanismos de transferência na camada limite próxima à superfície permeável.

Portanto, conclui-se que a modelagem numérica neste trabalho oferece uma ferramenta importante para descrição do problema convectivo mássico descrito por três equações de conservação.

Com base nesta tese de doutorado, pode-se sugerir os seguintes trabalhos seqüenciais:

(i) implentar uma malha computacional não-uniforme, em que o refinamento seja maior em regiões próximas à superfície permeável;

(ii) aprimorar o vínculo da modelagem ao processo de filtração tangencial utilizando-se a resistência devido à camada de concentração de polarização, $R_{p}$, como uma função;

(iii) implementar outros modelos de turbulência, como os modelos de uma equação e de duas equações $(k-\epsilon)$, bem como propor mudanças particulares, caso sejam 
necessárias, em modelos turbulentos para a camada limite turbulenta junto a uma superfície permeável. 


\section{Referências Bibliográficas}

[ACHESON, 1960] ACHESON, D. (1960). Elementary fluid dynamics. John Wiley \& Sons, Inc.

[ALHUMAIZI, 2004] ALHUMAIZI, K. (2004). Comparison of finite difference methods for the numerical simulation of reacting flow. Computers $\& 5$ Chemical Engeineering, 28:1759-1769.

[AlVES et al., 2003] AlVES, M. A., OliveIRA, P. J., and PINHO, F. T. (2003). A convergent and universally bounded interpolation scheme for the treatment of advection. International Journal for Numerical Methods in Fluids, 41:47-75.

[ARNOT et al., 2000] ARNOT, T. C., FIELD, R. W., and KOLTUNIEWICZ, A. B. (2000). Cross-flow and dead-end microfiltration of oily-water emulsions part ii. mechanisms and modelling of flux decline. Journal of Membrane Science, 169:1-15.

[BACCHIN et al., 2005] BACCHIN, P., ESPINASSE, B., and AIMAR, P. (2005). Distributions of critical flux: modelling, experimental analysis and consequences for cross-flow membrane filtration. Journal of Membrane Science, 250:223-234. 
[BARroso et al., 1987] BARroso, L. C., BARroso, M. M. A., CAMPOS Filho, F. F., CARVAlhO, M. L. B., and MAIA, M. L. (1987). Cálculo numérico (com aplicações). Editora Harbra Ltda, $2^{a}$ edição.

[BASHIR and REUSS, 1992] BASHIR, I. and REUSS, M. (1992). Dynamic model for cross-flow microfiltration of microbial suspensions in porous tubes. Chemical Engineering Science, 47(1):189-203.

[BENNETT and MYERS, 1978] BENNETT, C. O. and MYERS, J. (1978). Fenômenos de transporte: quantidade de movimento, calor e massa. McGraw-Hill.

[BERMAN, 1953] BERMAN, A. (1953). Laminar flow in channels with porous walls. Journal of Applied Physics, 24(9):1232-1235.

[BIRD et al., 1987] BIRD, R., ARMSTRONG, R., and HASSAGER, O. (1987). Dynamics of polymeric liquids. Wiley-Interscience Publication.

[BIRD et al., 1990] BIRD, R., STEWARD, W., and LIGHTFOOT, E. (1990). Transport phenomena. Oxford Applied Mathematics and Computing Science Series.

[BOWEN and WILLIANS, 2001] BOWEN, W. R. and WILLIANS, P. M. (2001). Prediction of the rate of cross-flow ultrafiltration of colloids with concentration-dependent diffusion coefficient and viscosity - teory and experiment. Chemical Engineering Science, 56:3083-3099.

[BRETAS and D'AVILA, 2000] BRETAS, R. and D'AVILA, M. A. (2000). Reologia de polímeros fundidos. Editora da UFSCar. 
[CARREAU et al., 1997] CARREAU, P., DE Kee, D., and CHHABRA, R. (1997). Rheology of polymeric systems - principles and applications. Hanser Publishers.

[CEBECI and BRADSHAW, 1984] CEBECI, T. and BRADSHAW, P. (1984). Physical and computational aspects of convective heat transfer. Springer-Verlag, New York, Inc.

[CHENG et al., 1997] CHENG, T. W., YEH, H. M., and GAU, C. T. (1997). Resistance analyses for ultrafiltration in tubular membrane module. Separation Science and Technology, 32(16):2623.

[CHERYAN, 1998] CHERYAN, M. (1998). Ultrafiltration and microfiltration - handbook. Technomic Publishing Co.,Inc.

[CHHABRA and RICHARDSON, 1999] CHHABRA, R. and RICHARDSON, J. (1999). Non-Newtonian flow in the process industries. Butterworth Heinemann.

[CLAPP, 1963] CLAPP, R. (1963). International development in heat transfer, 1961. International Heat Transfer Conference, New York, NY.

[CURCIO et al., 2001] CURCIO, S., CALABRÒ, V., and CINDIO, B. (2001). Fruit juice concentration by membranes: effect of rheological properties on concentration polarization phenomena. Journal of Food Engineering, 48:235-241.

[CZEKAJ et al., 2001] CZEKAJ, P., LÓPEZ, F., and GÜELL, C. (2001). Membrane fouling by turbidity constituents of beer and wine: characterization and prevention by means of infrasonic pulsing. Journal of Food Enginnering, 49:25-36. 
[DAMAK et al., 2004a] DAMAK, K., AYADI, A., ZEGHMATI, B., and SCHMITZ, P. (2004a). Concentration polarisation in tubular membranes a numerical approach. Desalination, 171:139-153.

[DAMAK et al., 2004b] DAMAK, K., AYADI, A., ZEGHMATI, B., and SCHMITZ, P. (2004b). Modeling of crossflow membrane separation processes under laminar flow conditions in tubular membrane. Desalination, 168:231-239.

[DAMAK et al., 2004c] DAMAK, K., AYADI, A., ZEGHMATI, B., and SCHMITZ, P. (2004c). A new navier-stokes and darcy's law combined model for fluid flow in crossflow filtration tubular membranes. Desalination, 161:67-77.

[DARU and TENAUD, 2001] DARU, V. and TENAUD, C. (2001). Evaluation of tvd high resolution schemes for unsteady viscous shocked flows. Computers \& Fluids, 30:89-113.

[FERREIRA, 2001] FERREIRA, V. G. (2001). Análise e implememtação de esquemas de convecção e modelos de turbulência para simulação de escoamentos incompressíveis envolvendo superfície livre. PhD thesis, ICMC-USP, São Carlos-SP.

[FERREIRA et al., 2007] FERREIRA, V. G., OISHI, C. M., KUROKAWA, F. A., KAIBARA, M. K., CUMinAtO, J. A., CASTELO, A., MANGIAVACCHI, N., TOMÉ, M. F., and McKEE, S. (2007). A combination of implicit and adaptative upwind tools for the numerical solution of incompressible free surface flows. Communications in $\mathrm{Nu}$ merical Methods in Engineering, 23:419-445.

[FORTUNA, 2000] FORTUNA, A. O. (2000). Técnicas computacionais para dinâmica de fluidos. EDUSP. 
[FOX and McDONALD, 1995] FOX, R. and McDONALD, A. (1995). Introdução à mecânica dos fluidos. Livros Técnicos e Ciênficos.

[FREIRE et al., 2002] FREIRE, A., MENUT, P., and SU, J. (2002). Turbulência. 1a Escola de Primavera em Transição e Turbulência, Rio de Janeiro.

[FROMM, 1968] FROMM, J. (1968). A method for reducing dispercion in convective difference schemes. Journal of Computational Physics, 3:217-223.

[GASKELL and LAU, 1988] GASKELL, P. and LAU, A. (1988). Curvature-compensated convective transport: Smart, a new boundedness-preserving transport algorithm. International Journal for Numerical Methods in Fluids, 8:617-641.

[GEKAS and HALLSTRÖM, 1987] GEKAS, V. and HALLSTRÖM, B. (1987). Mass transfer in the membrane concentration polarization layer under turbulent cross flow - i. critical literature review and adaptation of existing sherwood correlations to membrane operations. Journal of Membrane Science, 30:153-170.

[GERALDES et al., 2001] GERALDES, V., SEMIÃO, V., and PINHO, M. (2001). Flow and mass transfer modelling of nanofiltration. Journal Membrane Science, 191:109-128.

[HANEDA, 2006] HANEDA, R. (2006). Investigação do desempenho de diferentes estrutas microporosas tubulares na retenção de bactérias em suspensão por microfiltração tangencial. Master's thesis, EESC-USP, São Carlos-SP.

[HARLOW and WELCH, 1965] HARLOW, F. H. and WELCH, J. E. (1965). Numerical calculation of time-dependent viscous incompressible flow of fluid with free surface. Physics of Fluids, 8:2182-2189. 
[HIRT et al., 1975] HIRT, C. W., NICHOLS, B. D., and ROMERO, N. C. (1975). Sola - a numerical solution algorithm for transient fluid flows. Los Alamos Scientific Report $L A-5852$.

[HORNBECK et al., 1963] HORNBECK, R., ROULEAU, W., and OSTERLE, F. (1963). Laminar entry problem in porous tubes. The Physics of Fluid, 6(11):1649-1654.

[http://www.braseq.com.br/pdf/ brookfield.pdf, 2005] http://www.braseq.com.br/pdf/ brookfield.pdf (2005). Viscosidade e reologia.

[HUNG and PERNG, 1991] HUNG, C. and PERNG, Y. (1991). Flow of non-newtonian fluid in the entrance region of a tube with porous walls. International Journal of Heat and Fluid Flow, 12(3):263-268.

[KAPLAN, 1972] KAPLAN, W. (1972). Cálculo avançado. Ed. Edgard Blücher Ltda.

[KARODE, 2001] KARODE, S. (2001). Laminar flow in channels with porous walls, revisited. Journal of Membrane Science, 191:237-241.

[KARODE, 2000] KARODE, S. K. (2000). A new unsteady-state model for macromolecular ultrafiltration. Chemical Engineering Science, 55:1769-1773.

[KOTZEV, 1994] KOTZEV, T. (1994). Numerical study of the fluid dynamics and mass transfer of an ultrafiltration performance in a tube membrane module. International Journal of Engeering Science, 32(2):359-368.

[LAX and WENDROFF, 1960] LAX, P. and WENDROFF, B. (1960). Systems of conservations laws. Communications in Pure and Applied Mathematics, 13:217. 
[LEE and CLARK, 1997] LEE, Y. and CLARK, M. (1997). A numerical model of steadystate permeate flux during cross-flow ultrafiltration. Desalination, 109:241-251.

[LEE and CLARK, 1998] LEE, Y. and CLARK, M. (1998). Modeling of flux decline during crossfow ultrafiltration of colloidal suspensions. Journal of Membrane Science, 149:181202.

[LEONARD, 1979] LEONARD, B. (1979). A stable and accurate convective modeling procedure based on quadratic upstream interpolation. Computer Methods in Applied Mechanics and Engineering, 19:59-98.

[LEONARD, 1988] LEONARD, B. P. (1988). Simple hight-accuracy resolution program for convective modeling of discontinuities. International Journal for Numerical Methods in Fluids, 8:1291-1318.

[MACHADO, 2000] MACHADO, J. C. V. (2000). Reologia e escoamento de fluidos Ênfase na indústria do petróleo. Editora Interciência, R.J.

[MIGNARD and GLASS, 2001] MIGNARD, D. and GLASS, D. H. (2001). Fouling during the cross-flow ultrafiltration of protein: a mass-transfer model. Journal of Membrane Science, 186:133-143.

[MINNIKANTI et al., 1999] MINNIKANTI, V. S., DASGUPTA, S., and DE, S. (1999). Prediction of mass transfer coefficient with suction for turbulent flow in cross flow ultrafiltration. Journal of Membrane Science, 157:227-229.

[MUNSON-McGEE, 2002] MUNSON-McGEE, S. (2002). An approximate analytical solution for the fluid dynamics of laminar flow in a porous tube. Journal of Membrane Science, 197:223-230. 
[NAKAO et al., 1979] NAKAO, S., NOMURA, T., and KIMURA, S. (1979). Characteristics of macromolecular gel layer formed on ultrafiltration tubular membrane. AIChE Journal, 25(4):615-622.

[NASSEHI and PETERA, 1994] NASSEHI, V. and PETERA, J. (1994). A new leastsquares finite element model for combined navier-stokes and darcy flows in geometrically complicated domains with solid and porous boundaries. International Journal for Numerical Methods in Engineering, 37:1609-1620.

[PARIS et al., 2002] PARIS, J., GUICHARDON, P., and CHARBIT, F. (2002). Transport phenomena in ultrafiltration: a new two-dimensional model compared with classical models. Journal of Membrane Science, 207:43-58.

[PARVATIYAR, 1998] PARVATIYAR, M. G. (1998). Mass transfer in a membrane tube with turbulent flow of newtonian and non-newtonian fluids. Journal of Membrane Science, 148:45-57.

[PATANKAR, 1980] PATANKAR, S. (1980). Numerical heat transfer and fluid flow. Hemisphere Publishing Co.

[PRABHAKAR et al., 2000] PRABHAKAR, R., DASGUPTA, S., and DE, S. (2000). Simultaneous prediction of flux and retention for osmotic pressure controlled turbulent cross flow ultrafiltration. Separation and Purification Technology, 18:13-24.

[QUEIROZ, 2004] QUEIROZ, V. (2004). Estudo experimental do escoamento e da composição de mistura no processo de filtração tangencial de suspensões macromoleculares. Master's thesis, EESC-USP, São Carlos-SP. 
[RAHIMI et al., 2005] RAHIMI, M., MADAENI, S. S., and ABBASI, K. (2005). Cfd modeling of permeate flux in cross-flow microfiltration membrane. Journal of Membrane Science, 255:23-31.

[RANJAN et al., 2004a] RANJAN, R., DasGUPTA, S., and S., D. (2004a). Mass transfer coefficient with suction for laminar non-newtonian flow in application to membrane separations. Journal of Food Engineering, 64:53-61.

[RANJAN et al., 2004b] RANJAN, R., DasGUPTA, S., and S., D. (2004b). Mass transfer coefficient with suction for turbulent non-newtonian flow in application to membrane separations. Journal of Food Engineering, 65:533-541.

[RICHARDSON and NASSEHI, 2003] RICHARDSON, C. and NASSEHI, V. (2003). Finite element modelling of concentration profiles in flow domains with curved porous boundaries. Chemical Engineering Science, 58:2491-2503.

[RIPPERGER and ALTMANN, 2002] RIPPERGER, S. and ALTMANN, J. (2002). Crossflow microfiltration - state of the art. Separation and Purification Technology, 26:19-31.

[ROMA, 2003] ROMA, W. N. L. (2003). Fenômenos de transporte para engenharia. RiMa Editora.

[RUGGIERO and LOPES, 1997] RUGGIERO, M. A. G. and LOPES, V. L. R. (1997). Cálculo numérico - aspectos teóricos e computacionais. Pearson Education do Brasil Ltda, $2^{a}$ edição. 
[SCHMITZ et al., 1992] SCHMITZ, P., HOUI, D., and WANDELT, B. (1992). Hydrodynamic aspects of crossflow microfiltration. analysis of particle deposition at the membrane surface. Journal of Membrane Science, 71:29-40.

[SCHULZ, 2003] SCHULZ, H. E. (2003). O essencial em fenômenos de transporte. EESC - São Carlos, SP.

[SETHI and WIESNER, 1997] SETHI, S. and WIESNER, M. R. (1997). Modeling of transient flux in cross-flow membrane filtration incorporating multiple particle transport mechanisms. Journal of Membrane Science, 136:191-205.

[SHAH R.K, 1978] SHAH R.K, L. A. (1978). Laminar flow forced convection in ducts. Academic Press, New York.

[SHERWOOD et al., 1975] SHERWOOD, T., PIGFORD, R., and WILKE, C. (1975). Mass transfer. New York.

[SILVA et al., 2007] SILVA, J., Ferreira, V. G., Venezuela, L. A., and Fontes, S. R. (2007). Modelagem numérica do mecanismo de transferencia de massa em processos de filtração tangencial. Proceedings CIBIM 8 - 8vo Congreso Iberoamericano de Ingeniería Mecánica.

[SILVA and Fontes, 2005] SILVA, J. and Fontes, S. R. (2005). Numerical modeling of a newtonian and non-newtonian fluid flow in tubular membranes applied to the microfiltration processes. Proceedings COBEM 2005 - 18th International Congress of Mechanical Engineering.

[SILVA and Fontes, 2006] SILVA, J. and Fontes, S. R. (2006). Modelagem de um escoamento laminar e turbulento para filtração tangencial em membranas tubulares. 
Proceedings ENCIT 2006 - 11th Brazilian Congress of Thermal Engineering and Sciences.

[SISSOM and PITTS, 1979] SISSOM, L. E. and PITTS, D. R. (1979). Fenômenos de transporte. Editora Guanabara Dois S.A.

[SKELLAND, 1967] SKELLAND, A. H. P. (1967). Non-Newtonian flow and Heat transfer. John Wiley \& Sons, Inc.

[SONG et al., 2000] SONG, B. LIU, G. R., LAM, K. Y., and AMANO, R. S. (2000). On a higher-order bounded discretization scheme. International Journal for Numerical Methods in Fluids, 32:881-897.

[SPARROW and LIN, 1964] SPARROW, E. and LIN, S. (1964). Flow development in the hydrodynamic entrance region of tubes and ducts. The Physics of Fluid, 7(3):338-347.

[SREENIVAS et al., 2001] SREENIVAS, K., RAGESH, P., DASGUPTA, S., and DE, S. (2001). Modeling of cross-flow osmotic pressre controlled membrane separation processes under turbulent flow conditions. Journal of Membrane Science, 201:203-212.

[TANNEHILL et al., 1984] TANNEHILL, J., ANDERSON, D., and PLETCHER, R. (1984). Computational fluid mechanics and heat transfer. Taylor \& Francis.

[VENEZUELA and FONTES, 2007] VENEZUELA, A. and FONTES, S. (2007). Analytical-numerical modeling of convective laminar flow in a permeable tube associated with the cross-flow process - axial-convective-difusive. Proceedings DINCON 200\%, 6th Brazilian Conference on Dynamics, Control and Their, São José do Rio Preto, Brasil. 
[VENEZUELA et al., 2007] VENEZUELA, A., PÉREZ-GUERRERO, J. S., and FONTES, S. R. (2007). Hybrid modeling of convective laminar flow in a permeable tube associated with the crossflow process. Communications in Nonlinear Science and Numerical Simulation, doi:10.1016/j.cnsns.200\%.11.009.

[WATERSON and DECONINCK, 2007] WATERSON, N. and DECONINCK, H. (2007). Design principles for bounded higher-order convection schemes - a unified approach. Journal of Computational Physics, 224:182-207.

[WIJMANS et al., 1984] WIJMANS, J. G., NAKAO, S., and SMOLDERS, C. A. (1984). Flux limitation in ultrafiltration: osmotic pressure model and gel layer model. Journal of Membrane Science, 20:115-124.

[WIJMANS et al., 1985] WIJMANS, J. G., NAKAO, S., VAN DEN BERG, J. W., TROELSTRA, F. R., and SMOLDERS, C. A. (1985). Hydrodynamic resistance of concentration polarization boundary layers in ultrafiltration. Journal of Membrane Science, $22: 117-135$.

[YEH, 2002] YEH, H. (2002). Decline of permeate flux for ultrafiltration along membrane tubes. Desalination, 145:153-157.

[YEH et al., 2004] YEH, H., DONG, J., and SHI, M. (2004). Momentum balance analysis of flux and pressure declines in membranes ultrafiltration along tubular modules. Journal of Membrane Science, 241:335-345.

[YEH and WU, 1997] YEH, H. and WU, H. (1997). Membrane ultrafiltration in combined hollow-fiber module systems. Journal of Membrane Science, 124:93-105. 
[YEH et al., 2003] YEH, H., WU, H., and DONG, J. (2003). Effects of design and operating parameters on the declination of permeate flux for membrane ultrafiltration along hollow-fiber modules. Journal of Membrane Science, 213:33-44.

[YEH and CHENG, 1993a] YEH, H. M. and CHENG, T. W. (1993a). Osmotic-pressure model with permeability analysis for ultrafiltration in hollow-fiber membrane modules. Separation Technology, 3:91.

[YEH and CHENG, 1993b] YEH, H. M. and CHENG, T. W. (1993b). Resistance-in-series for membrane ultrafiltration in hollow fibers of tube-and-shell arrangement. Separation Science and Technology, 28(6):1341-1355.

[YOO and HARTNETT, 1974] YOO, S. and HARTNETT, J. (1974). Proceedings of the 5 th International Heat Transfer Congress, Tokyo.

[YUAN and FINKELSTEIN, 1956] YUAN, S. and FINKELSTEIN, A. (1956). Laminar pipe flow with injection and suction through a porous wall. Trans. ASME, 78:719-724.

[ZEMAN and ZYDNEY, 1996] ZEMAN, L. and ZYDNEY, A. (1996). Microfiltration and ultrafiltration - principles and applications. Marcel Dekker, Inc.

[ZHU, 1992] ZHU, J. (1992). On the higher-orde bounded discretization schemes for finite volume computations of incompressible flows. Computation Methods Aplication Mechanics Engineering, 98:345-360. 
Referências 


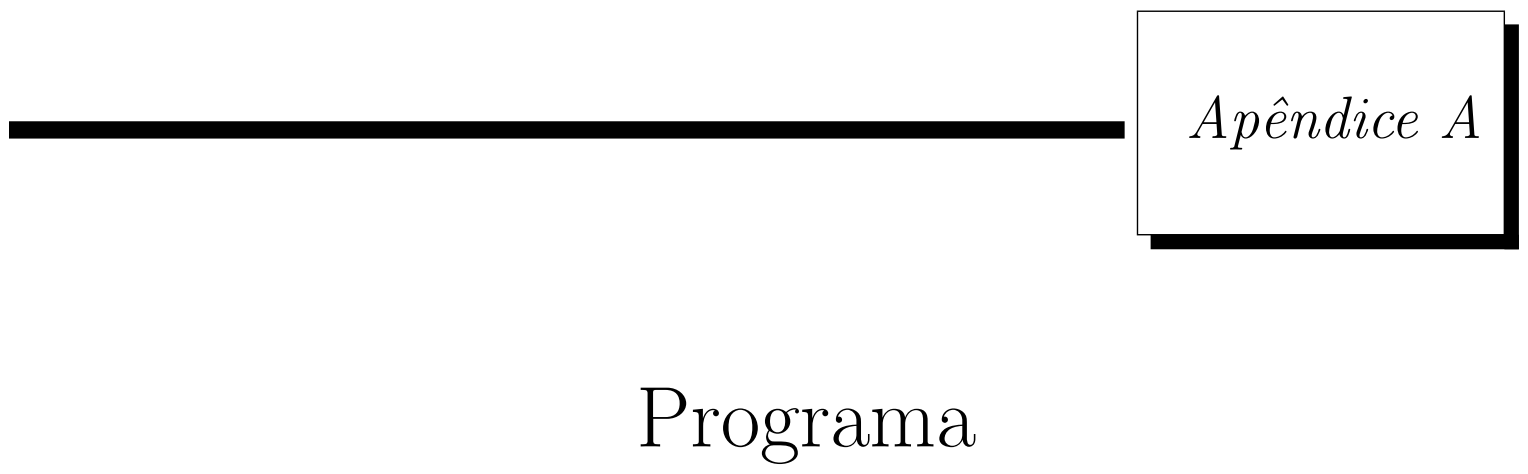

Esse apêndice é reservado para a apresentação da implementação do modelo matemático apresentado no capítulo 3. Devido a extensão do programa, optou-se por apresentar na forma de CD-Rom. 